Aus der Klinik und Poliklinik für Psychiatrie und Psychotherapie Ludwig-Maximilians-Universität München Direktor: Prof. Dr. med. H.-J. Möller

\title{
Der Einfluss kindlicher Traumatisierung auf eine spätere Drogenabhängigkeit
}

\author{
Dissertation \\ zum Erwerb des Doktorgrades der Medizin \\ an der Medizinischen Fakultät der \\ Ludwig-Maximilians-Universität zu München
}

\author{
vorgelegt von \\ Marion Schnieders
}

aus

Aachen

2005 


\section{Mit Genehmigung der Medizinischen Fakultät der Universität München}

1. Berichterstatter:

2. Berichterstatter:

Mitberichterstatter:

Mitbetreuung durch den

promovierten Mitarbeiter:

Dekan:

Tag der mündlichen Prüfung:
Prof. Dr. M. Soyka

Prof. Dr. Th. Gilg

Priv. Doz. Dr. R. Werth

Prof. Dr. W. v. Suchodoletz

Dr. med. M. Schäfer

Prof. Dr. D. Reinhardt

14.07.2005 


\section{Erklärung:}

Ich versichere ausdrücklich, dass ich die vorliegende Arbeit selbständig und ohne fremde Hilfe verfasst, andere als die von mir angegebenen Quellen und Hilfsmittel nicht benutzt und die aus den benutzten Werken wörtlich oder inhaltlich entnommenen Stellen einzeln nach Ausgabe (Auflage und Jahr des Erscheinens), Band und Seite des benutzten Werkes kenntlich gemacht habe, und dass ich die Dissertation bisher nicht einem Fachvertreter an einer anderen Hochschule zur Überprüfung vorgelegt oder mich anderweitig um die Zulassung zur Promotion beworben habe.

München, den 09.10.2004

Marion Schnieders 


\section{Inhaltsverzeichnis}

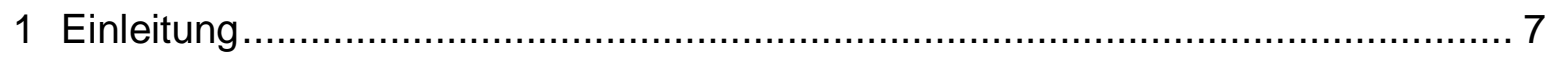

1.1 Kindliche Traumatisierung und Sucht ................................................. 7

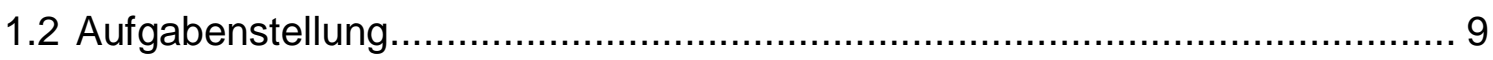

2 Zusammenhang von kindlicher Traumatisierung und Sucht in der Literatur......... 12

2.1 Kindliche Traumatisierung und Sucht ................................................. 12

2.1.1 Definition und Folgen einer kindlichen Traumatisierung .................... 12

2.1.2 Bisherige Studien und Erkenntnisse zum Zusammenhang zwischen kindlicher Traumatisierung und Sucht.......................................... 14

2.2 Definitionen von Missbrauchsarten und Sucht ........................................ 17

2.2.1 Definition sexueller Missbrauch .............................................. 17

2.2.2 Definition körperliche Misshandlung ........................................... 22

2.2.3 Definition Vernachlässigung ................................................. 24

2.2.4 Definition familiäre Gewalt ........................................................ 25

2.2.5 Definition emotionale Misshandlung ............................................ 25

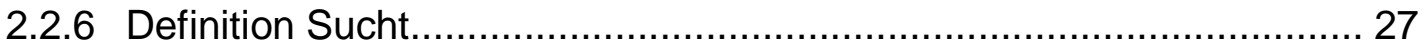

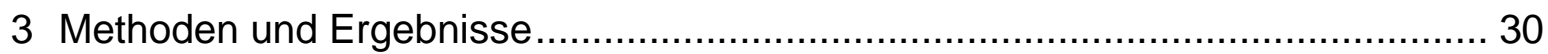

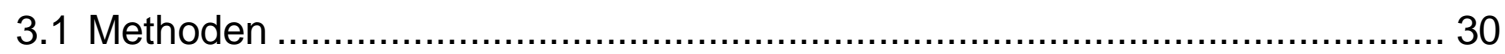

3.1.1 Auswahl der Stichprobe ............................................................ 30

3.1.2 Beschreibung des Interviews "European Addiction Severity Index" (EuropASI) ........................................................................ 31

3.1.3 Beschreibung des Fragebogens „Symptom Checkliste“ (SCL 90-R) ... 34

3.1.4 Beschreibung des Münchener Traumafragebogens ....................... 37

3.1.5 Datenverarbeitung und statistische Auswertung ............................ 39

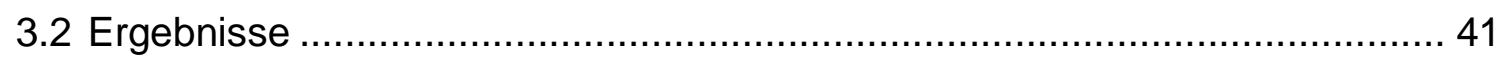

3.2.1 Soziodemographische Ergebnisse zur Stichprobe .......................... 41

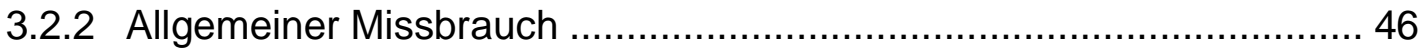

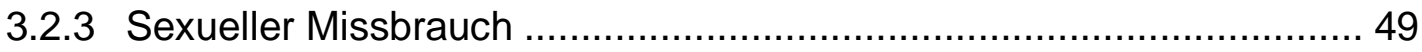

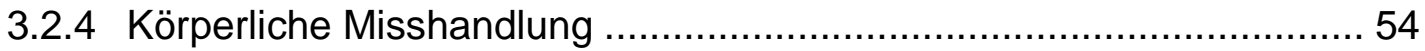

3.2.5 Vernachlässigung ................................................................... 58 


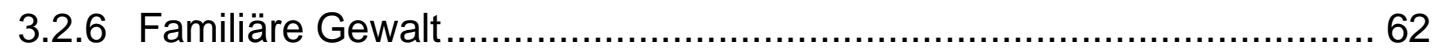

3.2.7 Emotionale Misshandlung.............................................................. 66

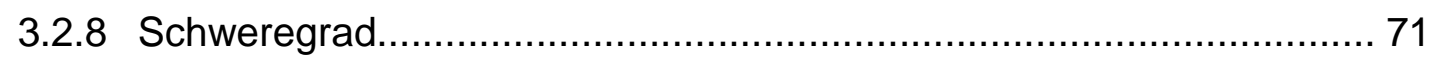

3.2.8.1 Schweregrad allgemeiner Missbrauch …………………....... 71

3.2.8.2 Schweregrad sexueller Missbrauch ...................................... 74

3.2.8.3 Schweregrad körperlicher Gewalt ........................................ 76

3.2.8.4 Schweregrad Vernachlässigung ......................................... 80

3.2.8.5 Schweregrad familiäre Gewalt ........................................ 82

3.2.8.6 Schweregrad emotionaler Missbrauch ................................... 86

3.2.9 Absolute Belastung der Missbrauchsgruppen .................................. 90

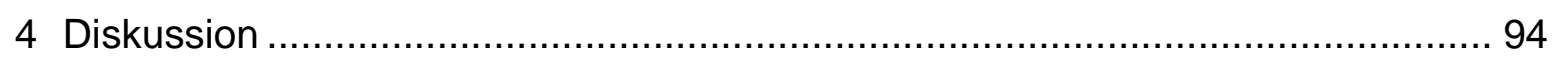

4.1 Diskussion des Einflusses von Missbrauch auf den Krankheitsverlauf .......... 94

4.1.1 Einfluss von Missbrauch auf die Entwicklung spezifischer

Symptome und die Suchtschwere ................................................ 94

4.1.1.1 Übersicht des Einflusses von Missbrauch auf die Problembereiche nach EuropASI ......................................... 95

4.1.1.2 Einfluss von Missbrauch auf die Entwicklung körperlicher

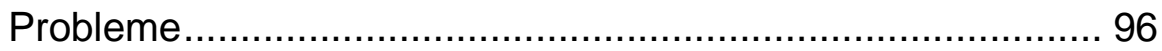

4.1.1.3 Einfluss von Missbrauch auf die Entwicklung psychischer Probleme (SCL 90-R) ..................................................... 97

4.1.1.4 Einfluss des sexuellen Missbrauchs auf die Entwicklung psychischer Probleme. 98

4.1.1.5 Einfluss der Vernachlässigung auf die Entwicklung psychischer Probleme. 99

4.1.1.6 Einfluss der körperlichen Misshandlung auf die Entwicklung psychischer Probleme..................................................... 100

4.1.1.7 Einfluss des Missbrauchs auf die Suchtschwere ................. 101

4.1.2 Diskussion der breit angelegten psychischen Symptombelastung .... 102

4.1.3 Diskussion der Ursächlichkeit der Symptome ................................. 104

4.1.4 Diskussion des Zusammenhangs zwischen Missbrauchsart und Stärke der Symptombelastung. 105 
4.2 Einfluss des Schweregrades des Missbrauchs auf den Krankheitsverlauf... 109

4.3 Einfluss geschlechtsspezifischer Unterschiede auf den Krankheitsverlauf .. 112

4.4 Ansatz einer Gruppenbildung unter Berücksichtigung der Missbrauchsart, des Schweregrads und des Geschlechts für eine differenzierte Therapie.... 115

4.5 Überprüfung der Stichprobe und Methodenkritik ................................... 118

4.5.1 Vergleich der Zusammensetzung dieser Stichprobe mit der Literatur 118

4.5.2 Vergleich der Ergebnisse zur Prävalenz von Kindesmissbrauch in der Literatur

4.5.3 Überprüfung der Ergebnisse des SCL 90-R durch einen Vergleich mit Referenzwerten von Franke................................................ 125

4.5.4 Methodenkritik .................................................................. 127

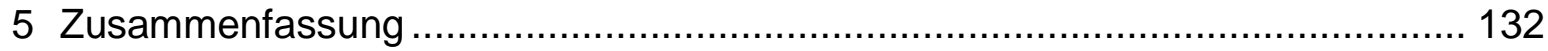

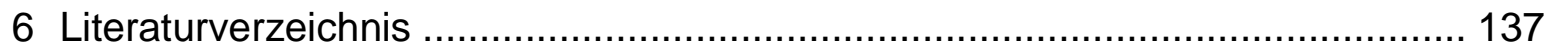

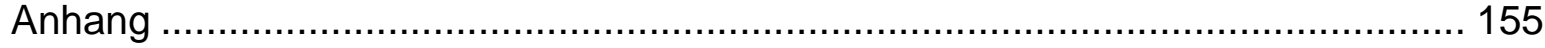

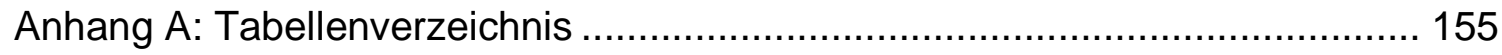

Anhang B: Münchener Traumafragebogen................................................ 160

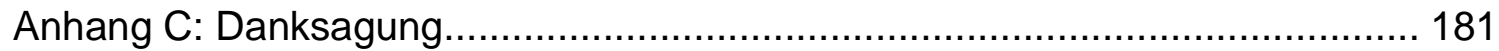




\section{Einleitung}

\subsection{Kindliche Traumatisierung und Sucht}

In Deutschland gibt es ca. 3,5 Millionen Menschen mit einer Abhängigkeit von psychotropen Substanzen wie Alkohol, Medikamente und illegale Drogen (Soyka 1997, 1998). Die Therapie der Drogenabhängigkeit erweist sich meist als schwierig, die Erfolge in Form von langfristigen Abstinenzraten liegen unter $30 \%$ (Übersicht bei Soyka 1998). Wesentlich für den Erfolg einer Therapie ist die Ergründung der Ursachen für die Drogenabhängigkeit. Gerade für die Gruppe der Drogensüchtigen besteht ein hoher Informationsbedarf über erfolgte Traumatisierungen und spätere Folgen. Die Ursachen der Drogenabhängigkeit sind nach bisherigem Wissensstand multifaktoriell zu betrachten. Realtraumatisierungen in der Kindheit scheinen die Häufigkeit späterer Suchterkrankungen aber zu erhöhen. Eine der schwerwiegensten Formen der Traumatisierung stellt der Kindesmissbrauch und insbesondere der sexuelle Kindesmissbrauch dar, deren Auswirkungen auf eine spätere Drogensucht in der vorliegenden Arbeit analysiert werden soll.

Auf Grund der Tabuisierung des Themas Kindesmissbrauchs liegen für die Häufigkeit von sexuellem Missbrauch in der Allgemeinbevölkerung nur sehr ungenaue Schätzungen vor. In Deutschland ist sexueller Missbrauch das häufigste registrierte Sexualdelikt. Im Jahr 1990 wurden ungefähr 12.800 sexuelle Missbrauchsdelikte angezeigt. Nach Dunkelfeldschätzungen ergeben sich Zahlen von jährlich ca. 300.000 missbrauchten Kindern in Deutschland (siehe Endres und Scholz, 1994). Das „National center of child abuse and neglect“ in den USA ging 1993 von ca. 200.000 jährlichen Missbräuchen bei einer wahrscheinlich noch viel höheren Dunkelziffer aus (Muram 1993). Nach Besharov (1993) schätzt man dort die Zahl körperlich misshandelter Kinder pro Jahr auf ca. 300.000 mit ca. 140.000 sexuellen Missbrauchsopfern. 
Eine Vielzahl von Studien weiste auf enge Korrelationen zwischen dem Vorhandensein einer Suchterkrankung oder einer sonstigen psychiatrischen Erkrankung (Essstörungen, Depressionen, Borderline-Störungen) und in der Kindheit stattgefundenem Missbrauch hin. Die Mehrheit der Studien konzentrierte sich dabei auf den sexuellen Kindesmissbrauch und seine Folgen (Moncrieff et al. 1996, Sullivan et al. 1995, Kinzl und Biebl 1991, Vize and Cooper 1995, Singer et al. 1989, Faller 1989, Stein et al. 1988, Cavaiola and Schiff 1988, Cohen and Densen-Gerber 1982, Kinsey 1953). Nach den bisherigen Erkenntnissen hat ein sexueller Missbrauch ein ca. vierfach erhöhtes Risiko für die spätere Entwicklung psychiatrischer Erkrankungen sowie ein ca. dreifach erhöhtes Risiko für Suchterkrankungen zur Folge (Saunders et al., 1992; Scott, 1992; Mullen et al., 1993). Auch für körperliche Misshandlungen (Kolko 1992) und für psychische Vernachlässigung in der Kindheit (Briere and Runtz 1990) konnten ähnliche Risikopotenziale festgestellt werden.

Ein monokausaler Zusammenhang des Kindesmissbrauchs mit einem spezifischen psychiatrischen Krankheitsbild konnte aber bisher nicht nachgewiesen werden (siehe bei Ernst, 1997). Nach Ulrich (1988) erhöht sexueller Missbrauch als Risikofaktor allein genommen die Wahrscheinlichkeit späterer psychischer Erkrankungen nicht. Bei Egle et al. (1997) findet man sexuellen Missbrauch sogar nur an 13. Stelle in der Häufigkeit von insgesamt 18 erfassten Risikofaktoren für psychische Erkrankungen. Missbrauchserlebnisse können daher kaum getrennt von anderen Faktoren in ihren eventuellen Auswirkungen interpretiert werden.

Auch wenn nach dem bisherigen Forschungsstand nicht von einem monokausalen Zusammenhang zwischen Kindesmissbrauch und Drogensucht auszugehen ist, so ist der Einfluss der Traumatisierung durch den Missbrauch in der Kindheit auf die Schwere und den Verlauf der Drogenerkrankung evident. Tatsächlich konnte kürzlich ein Zusammenhang zwischen frühkindlichem Missbrauch und der Schwere einer späteren Drogenerkrankung gezeigt werden 
(Schäfer et al. 2000). Die Zusammenhänge zwischen Missbrauch und späterer Drogenabhängigkeit sowie Unterschiede in der Suchtentwicklung zwischen Patientengruppen mit und ohne Missbrauchsvorgeschichte wurden in dieser Studie untersucht. Es zeigte sich, dass eine sexuelle Missbrauchsvorgeschichte signifikant gehäuft zusammen mit Missbrauch illegaler Drogen oder Medikamente, im Vergleich zu Alkohol auftrat. Vermehrter Gebrauch harter illegaler Drogen war mit schwerem sexuellen Missbrauch assoziiert. Es fand sich weiterhin in der Gruppe der sexuell Missbrauchten eine signifikante Häufung bestimmter Symptome: erhöhte Autoaggression, erhöhte Suizidneigung, Weglauftendenzen als Kind, soziale Isolation und Außenseitergefühle, Tendenz zu gehäufter Reviktimisierung, Prostitution und erhöhte Gewaltbereitschaft. Die Auswirkungen des Missbrauchs zeigten sich durch einen ungünstigeren Verlauf und schlechtere Prognose der späteren Suchterkrankung bei gehäufter Abhängigkeit von harten illegalen Drogen und erhöhter Symptombelastung. Basierend auf den Ergebnissen der Vorarbeit von Schäfer et al. wurde die Aufgabenstellung der vorliegenden Arbeit formuliert.

\subsection{Aufgabenstellung}

Das Ziel der vorliegenden Arbeit war es, den Einfluss der kindlichen Traumatisierung auf den Verlauf einer späteren Drogenabhängigkeit zu untersuchen. Die folgenden konkreten Fragestellungen sollten mit der Studie beantwortet werden:

- Kernfrage war, wie sich ein Missbrauch auf den Krankheitsverlauf der Drogenerkrankung auswirkt. Ziel war es dabei zu überprüfen, ob die These zutrifft, dass ein sexueller Missbrauch allgemein mit einem schlechteren Verlauf der Suchterkrankung und höherer Symptombelastung assoziiert ist (vgl. hierzu auch Vorarbeiten Schäfer et al. 2000). Auch Rounsaville et al. wiesen schon 1982 darauf hin, dass gerade die Gruppe der Abhängigen mit Kindheitstrauma (Gewalt, sexueller Missbrauch) später 
eine besonders auffällige Psychopathologie und vergleichsweise die niedrigsten sozialen Kompetenzen zeigt. Diese Gruppe schien außerdem die schlechteste therapeutische Prognose zu haben.

- Weiterhin sollte analysiert werden, ob die Art des Missbrauchs (körperliche Misshandlung, sexueller Missbrauch, emotionaler Missbrauch, Vernachlässigung, familiäre Gewalt) die Ausprägung der späteren Drogenabhängigkeit (Verlauf, unterschiedliche Symptombelastung) beeinflusst und ob sich dadurch Ansätze für unterschiedliches Suchtverhalten ergeben.

- Auch die Schwere des Missbrauchs sollte für eine Beurteilung herangezogen werden: So konnten beispielsweise Mullen et al. (1993) nur bei sexuellem Missbrauch mit vollzogenem Koitus ein statistisch gehäuftes Auftreten von Suizidhandlungen, Essstörungen oder stationären Behandlungen in psychiatrischen Kliniken gegenüber der Normalbevölkerung finden. Der Schweregrad des sexuellen Missbrauchs schien also einen erheblichen Einfluss auf die psychiatrische Erkrankung haben. Aus diesem Grund haben wir in dieser Arbeit eine Unterteilung der Missbrauchsformen in Schweregrade in Anlehnung an McGee (1995) vorgenommen. Diese Unterteilung des Missbrauchs in Schweregrade findet sich in dem hierfür erstellten Traumafragebogen. Ziel war es also die Frage zu beantworten, welche spezifischen Auswirkungen die Schwere des Missbrauchs auf den Krankheitsverlauf hat.

- In der Arbeit war ferner zu berücksichtigen, ob sich bei den Auswirkungen des Missbrauchs auf den Krankheitsverlauf Unterschiede zwischen den Geschlechtern finden lassen.

- Letztlich sollte in Analogie zu Krausz und Müller-Thomsen (1994) sowie Krausz und Freyberger (1997) untersucht werden, ob sich Untergruppen 
aufgrund von Missbrauchserfahrungen bezüglich komorbider psychischer Störungen, Substanzmissbrauch und Realtraumatisierungen bilden lassen, um so in weiterführenden Arbeiten differentielle Behandlungsstrategien entwickeln zu können.

Zur Beantwortung der Fragestellungen wurde dazu eine Studie mit ca. 200 drogen- und alkoholabhängigen Patienten in mehreren psychiatrischen Kliniken durchgeführt, bei der die Patienten zu ihrer Sucht sowie möglichen Missbrauchsvorfällen in der Kindheit befragt wurden. In einer parallelen Doktorarbeit wurden die Ergebnisse der Korrelation von Missbrauch und Sucht bei alkoholabhängigen Patienten beschrieben. Die vorliegende Arbeit fasst die Ergebnisse der Zusammenhänge von Missbrauch und Sucht für drogenkranke Patienten zusammen. 


\section{Zusammenhang von kindlicher Traumatisierung und Sucht in der Literatur}

\subsection{Kindliche Traumatisierung und Sucht}

\subsubsection{Definition und Folgen einer kindlichen Traumatisierung}

Die Traumatisierung ist der übergeordnete Aspekt der in dieser Arbeit diskutierten Missbrauchsformen des sexuellen Missbrauchs, körperlicher Misshandlung, Vernachlässigung, des emotionalen Missbrauchs und familiärer Gewalt. Der Begriff „Trauma" fand im 19. Jahrhundert zunächst Verwendung für eine durch äußere Verletzung entstandene Wunde. Auch heute wird dieser Begriff noch in der Unfallmedizin und Chirurgie benutzt. Erst 1895 konzipierten Freud und Breuer in ihren Studien zur Hysterie den Begriff des psychischen Traumas. Ausgangspunkt waren hysterische Erkrankungen, denen das Trauma sexueller Verführung - wie Freud es damals nannte - zugrunde lag. So fand die bis heute am häufigsten benannte Form der Traumatisierung, der sexuelle Missbrauch, erstmalig Zugang in die psychotherapeutische Behandlung.

Allgemein sowohl für Kinder als auch Erwachsene ist ein Trauma ein plötzliches, intensives, gewalttätiges und schmerzhaftes Ereignis, das die psychischen Verarbeitungsmöglichkeiten eines Menschen überfordert, weil es die Psyche gleichsam überschwemme (Shengold 1995). Schwere Unfälle, Misshandlungen und sexueller Missbrauch seien Prototypen dieses Geschehens, die ein Übermaß an Stimulierung bieten. Aber auch das Gegenteil, ein Mangel an Stimulierung, der das erträgliche Maß übersteigt, wie er z. B. bei Deprivationen vorliegt, wirke traumatisierend und hemme ebenfalls die normale Entwicklung (Shengold 1995). Sowohl ein Übermaß an Stimulierung als auch deren Mangel können folglich traumatisierend wirken. Sie haben einen Rückzug vom Körpergefühl und von den Emotionen zur Folge und erzwingen Abwehrmaßnahmen gegenüber der schrecklichen Gefühlsintensität. Die fünf 
Missbrauchsformen, die in dieser Arbeit betrachtet wurden, sexueller Missbrauch, körperliche Misshandlung, Vernachlässigung, emotionaler Missbrauch und familiäre Gewalt, beinhalten dieses beschriebene Übermaß bzw. diesen Mangel an Stimulierung.

Für das Verständnis der in dieser Arbeit betrachteten kindlichen Traumatisierung greifen wir auf die Ergebnisse von Leonore Terr (1995) zurück. Terr unterschied auf Basis der unterschiedlichen Auswirkungen der Traumatisierung zwischen Kindern mit einem einmaligen Trauma (Trauma-Opfer Typ I) und wiederholt traumatisierten Kindern (Trauma-Opfer Typ II). Sie spricht vom Trauma-Opfer Typ I bei den Kindern und Jugendlichen, die ein einmaliges traumatisches Erlebnis, ein Schocktrauma, gehabt haben. Für unsere Arbeit ist dagegen in der Regel der Typ II des Trauma-Opfers maßgebend, der wiederholten Traumatisierungen ausgesetzt ist. Diese Trauma-Opfer des Typ II müssen massive Schutzmechanismen gegen das Erinnern aufbauen, weil darin für sie die einzige Möglichkeit besteht, ihre schreckliche Kindheit zu überstehen. Häufig sind Depersonalisation und Dissoziation die Folge. Typ II Traumata sind die über eine längere Zeit anhaltenden Traumatisierungen wie physische lebensbedrohliche Erkrankungen innerhalb der Familie, psychische Erkrankung innerhalb der Familie, emotionale und körperliche Verwahrlosung, Arbeitslosigkeit mit einhergehender Vernachlässigung und Gewalterfahrung, anhaltender emotionaler Missbrauch, anhaltender sexueller Missbrauch und körperliche Misshandlung, anhaltende Kriegs- und Terrorerfahrung, generationsübergreifende Traumatisierungen. In dem Aufbau der Schutzmaßnahmen gegen die Traumatisierung liegt das Verständnis für die Auswirkungen auf den Krankheitsverlauf der Patienten.

Eine starke Traumatisierung des Kindes kann nach Finkelhor (Finkelhor 1984, Finkelhor \& Browne 1985) schwerwiegende Folgen wie selbstdestruktive Akte, Angst- und Panikattacken, Dissoziation, Zwang, Phobien und Sucht haben. Allerdings entscheiden letztlich die Art, die Schwere der Traumatisierung, die 
Kombination mit zusätzlichen Risikofaktoren, aber auch die kompensatorischen Einflüsse in Form von Schutzfaktoren darüber, welche Langzeitfolgen entstehen.

\subsubsection{Bisherige Studien und Erkenntnisse zum Zusammenhang zwischen kindlicher Traumatisierung und Sucht}

Die Traumatisierung in der Kindheit und Jugend durch sexuellen sowie körperlichen Missbrauch stand in den letzten Jahren im Fokus vieler Studien. Diese Studien zeigten ein gehäuftes Vorkommen von kindlichem sexuellem Missbrauch unter Patienten mit bestimmten psychischen und psychosomatischen Erkrankungen (Bryer et al. 1987, Bulik et al. 1989, Coons 1986, Damlouji und Ferguson 1995, Finkelhor und Browne 1995, Friedmann et al. 1982, Gold 1986, Greenwald et al. 1990, Herman 1994, Lindberg und Distad 1985, Morrison 1989, Ogata et al. 1990, Sloan und Leichner 1986).

Der Ergründung der Zusammenhänge zwischen Missbrauch und Sucht wurde in der Literatur intensiv nachgegangen (Moncrieff et al. 1996, Sullivan et al. 1995, Kinzl und Biebl 1991, Vize und Cooper 1995, Singer et al. 1989, Faller 1989, Stein et al. 1988, Cavaiola and Schiff 1988, Cohen and Densen-Gerber 1982, Kinsey 1953). Die Ergebnisse weisen darauf hin, dass das Risiko einer Sucht sich auf Grund des Missbrauchs im Kindes- und Jugendalter erhöht.

Substanzmittelmissbrauch als Folgeerscheinung bei Opfern sexuellen Missbrauchs und körperlicher Misshandlung war bei den Studien von Rose et al. (1991) in erhöhtem Maße zu finden. Ebenso zeigten bei Brown und Anderson (1991) stationäre Patienten mit einer körperlichen und sexuellen Missbrauchsvorgeschichte eine höhere Rate von Suchterkrankungen als Patientinnen und Patienten ohne eine solche Vorgeschichte. Wenn ein elterlicher Substanzmittelgebrauch vorlag, bestand nach Yama et al. (1993) bei den Jugendlichen ein doppelt so hohes Risiko für einen höheren symptomatologischen Ausprägungsgrad von psychiatrischen Erkrankungen. Eine 
hohe Korrelation zwischen Traumatisierung in der Kindheit und Substanzmissbrauch konnten auch Triffleman et al. (1995) nachweisen.

Downs et al. (1998) stellten ebenfalls fest, dass ein sexueller oder körperlicher Missbrauch in der Kindheit ein erhöhtes Risiko für eine spätere Drogenabhängigkeit nach sich zieht. In einer Befragung von 4790 Studenten in Washington fanden Bensley et al. (1999) bei vorliegendendem Missbrauch unter diesen Studenten eine erhöhte Wahrscheinlichkeit für Drogeneinnahme. Am höchsten war die Wahrscheinlichkeit einer Drogeneinnahme bei den Studenten, die eine körperliche Misshandlung in Kombination mit sexuellem Missbrauch (entspricht der hier verwendeten mittleren Schwere des sexuellen Missbrauchs) erlitten hatten (12 mal höher als die Mitstudenten ohne Missbrauchserfahrung).

Interessant ist, dass nach Egle et al. (1997) sexueller Missbrauch lediglich an 13. Stelle in der Häufigkeit von insgesamt 18 erfassten Risikofaktoren für psychische Erkrankungen steht. Allerdings ist dabei die Art des sexuellen Missbrauchs von großer Bedeutung: "Sexueller Missbrauch mit vollzogenem Koitus lässt ein statistisch gehäuftes Auftreten von Suizidhandlungen, Essstörungen oder stationären Behandlungen in psychiatrischen Kliniken gegenüber der Normalbevölkerung finden“ (Mullen et al. 1993). Mullen et al. beschrieben bei schwerem sexuellem Missbrauch ein vierfach höheres Risiko für die Entwicklung psychiatrischer Erkrankungen (vgl. auch Saunders et. al. 1992, Scott 1992). Die Arbeiten von Mullen et al. waren u.a. Motivation für die Berücksichtigung der Missbrauchsschwere in dieser Arbeit, um einen differenzierteren Blick auf die Auswirkungen von Missbrauch zu erlauben. Die Definition bei Mullen entspricht der in dieser Arbeit verwendeten Definition des „schweren sexuellen Missbrauchs“.

Aus den oben angeführten Studien wird allgemein geschlossen, dass das Risiko einer psychiatrischen Erkrankung, z.B. der Suchterkrankung, nach einer Missbrauchserfahrung in der Kindheit steigt. Ein monokausaler Zusammenhang 
zwischen dem Missbrauch, hier insbesondere dem sexuellen Missbrauch, und einer späteren Erkrankung konnte aber noch nicht nachgewiesen werden (Ernst 1997). Dies bestätigen auch Untersuchungen an Opfern von Missbrauch, die keine Symptome aufweisen. Im Rahmen einer kinderärztlichen Untersuchung stellten Caffaro-Rouget, Lang und van Santen (1989) fest, dass bei ihrer Erhebung 49\% der Kinder trotz Missbrauchs im Normalbereich lagen. Zu ähnlichen Ergebnissen kamen Tong, Oates und McDowell (1987) bei denen 36\% trotz Missbrauchs keine Symptome zeigten. Auch Conte und Schuerman (1987) wiesen darauf hin, dass $21 \%$ ihrer Stichproben überhaupt keine Symptome zu zeigen schienen.

Die These, dass sexueller Missbrauch die alleinige Ursache einer Suchtentwicklung sein könnte, wurde allgemein verlassen. Nach Ulrich (1988) ist von einem nichtlinearen, exponentiellen Zusammenwirken der einzelnen Risikofaktoren auszugehen. Während die Wirkung eines einzelnen Faktors eher gering ist, erhöht sich bei bereits zwei Faktoren die Wahrscheinlichkeit, dass eine Entwicklungsstörung auftritt um das Vierfache. Schutzfaktoren können dabei die Wirkung der Risiko- oder Traumafaktoren verhindern oder eingetretene Schäden wieder ausgleichen.

Einen Zusammenhang zwischen Missbrauch und einem schlechteren Krankheitsverlauf bei drogenkranken Patienten stellten Schäfer et al. (2000) fest. Sie befragten 100 Suchtkranke in Hinblick auf Art und Häufigkeit von Gewalt- und sexuellen Missbrauchserfahrungen bis zum 16. Lebensjahr. 60\% der Befragten wurden vor ihrem 16. Lebensjahr sexuell missbraucht. Bei 40\% der Männer und $50 \%$ der Frauen lag dabei ein „schwerer Missbrauch“ vor. Unter den drogenkranken Patienten konnte eine signifikante Häufung von sexuellem Missbrauch in der Kindheit nachgewiesen werden. Ein vermehrter Gebrauch harter illegaler Drogen war mit schwerem sexuellen Missbrauch assoziiert. Es konnte aber auch kein kausaler Zusammenhang zwischen sexuellem Missbrauch in der Kindheit und späterer Polytoxikomanie festgestellt werden. Die 
Auswirkungen des sexuellen Missbrauchs in der Kindheit zeigten sich eher in Form eines ungünstigen Verlaufes und einer schlechteren Prognose der späteren Suchterkrankung bei gehäufter Abhängigkeit von harten illegalen Drogen und erhöhter Symptombelastung. Die Ergebnisse von Schäfer et al. (2000) bildeten für uns die Grundlage für die Fragestellungen dieser Arbeit. Die Fragestellung nach spezifischen Symptomen, einem schlechteren Verlauf der Suchterkrankung sowie dem Zusammenhang mit Art und Schwere des Missbrauchs sollen aufbauend auf der Arbeit von Schäfer vertieft werden.

\subsection{Definitionen von Missbrauchsarten und Sucht}

Im Folgenden werden die Definitionen der wichtigsten Begriffe dieser Arbeit hergeleitet. Die Schwierigkeit der Definition von Missbrauch soll exemplarisch an Hand des sexuellen Missbrauchs gezeigt werden. Für die anderen Missbrauchsarten gilt die Problematik analog, es wird aber auf eine entsprechende Ausführlichkeit der Definitionsherleitung verzichtet.

\subsubsection{Definition sexueller Missbrauch}

Amann und Wipplinger (1997) haben sowohl eine umfangreiche Übersicht der vorherrschenden Definitionen als auch Kriterien für ein Vorliegen bzw. eine Beschreibung der Art des sexuellen Missbrauchs zusammengestellt. Für ein besseres Verständnis der Unterschiede, aber auch der Gemeinsamkeiten der Definitionen, soll zunächst auf diese potenziellen Faktoren für die Definition des sexuellen Missbrauchs eingegangen werden, bevor die Auswahl und Gewichtung dieser Faktoren anhand einiger Definitionen beschrieben wird. Als Faktoren sind zu nennen:

- Altersdifferenz zwischen Opfer und Täter (Conte 1993, Bange 1992, Bange \& Deegener 1996, Gaenslen-Jordan et al. 1990, Richter-Appelt 1994)

- Art der sexuellen Handlung. Invasive Praktiken wie Penetration können als besonders schädigend gelten (Barnett et al. 1993) 
- Absicht des Täters

- Bedürfnisbefriedigung des Mächtigeren (Conte 1993, Gaenslen-Jordan et al. 1990, Richter-Appelt 1994)

- Folgen des Missbrauchs

- Missachtung des kindlichen Willens

- Sich missbraucht fühlen

- Sexueller Missbrauch durch Blicke und Worte

- Zwang und Gewalt. Besonders zerstörerisch wirkt sich die Verbindung mit körperlicher Gewalt und Misshandlung aus (Barnett et al. 1993)

- Geheimhaltung (Gaenslen-Jordan et al. 1990, Richter-Appelt 1994)

- Häufigkeit bzw. Chronizität (Barnett et al. 1993)

- Alter des Kindes (Barnett et al. 1993, Thornberry et al. 2001)

- Entwicklungskontext des Kindes, z.B. Heim oder Familien, wobei die Folgen gravierender sind, wenn der Missbrauch von den Bezugspersonen des Kindes ausgeübt wird (Barnett et al. 1993)

- Die Person des Täters, z.B. Verwandtschafts- oder Bekanntschaftsgrad. Die übelsten Folgen ergeben sich bei Inzest durch die engsten erwachsenen Bezugspersonen wie Vater, Mutter oder Stiefeltern (Barnett et al. 1993)

Je nach Autor werden die genannten Kriterien selektiert und gewichtet, so dass in der aktuellen Literatur eine Vielzahl von Definitionen vorliegen. In der Varianz der Definitionen liegt einer der hauptsächlichen Gründe, dass die Prävalenz des sexuellen Missbrauchs in den verschiedenen Studien sehr unterschiedlich ausfallen kann. Im folgenden werden einige wichtige Ansätze für eine Definition des sexuellen Missbrauchs beschrieben.

Nach Engfer (1992) versteht man unter sexueller Kindesmisshandlung die Beteiligung von Kindern und noch nicht ausgereiften Jugendlichen an sexuellen Aktivitäten (z.B. Entblößen und Berühren der Genitalien, vaginale, orale und anale Penetration, Herstellung pornographischen Materials, Anleitung zur 
Prostitution), denen sie aufgrund ihres Entwicklungsstandes nicht verantwortlich zustimmen können. Dabei missbraucht der in der Regel männliche Erwachsene oder Jugendliche das vorhandene Macht- und Kompetenzgefälle zu seinem eigenen Nutzen. Kommt es innerhalb der Kernfamilie zu sexueller Kindesmisshandlung, so wird dafür der Begriff Inzest verwendet (Moggi 1991).

Autoritäts- und Gewaltstrukturen, die der Erwachsene im Umgang mit den Kindern zur Verfügung hat, stehen in der "gesellschaftlichen Definition“ im Vordergrund (Geier 1990, Brockhaus und Kolshorn 1993).

Bei der „feministischen Definition“ wird besonders betont, dass der sexuelle Missbrauch gegen weibliche Opfer gerichtet ist (Kavemann und Lohstöter 1986, Draijer 1990). Gemeinsam steht bei der gesellschaftlichen und der feministischen Definition das Macht- und Autoritätsverhältnis zwischen Täterln und Opfer im Vordergrund. Bei der feministischen Definition wird dieses durch die geschlechtliche Zuweisung von männlichen Tätern und weiblichen Opfern ergänzt.

Bei der „entwicklungspsychologischen Definition“ werden die entwicklungsbedingten Faktoren des Kindes hervorgehoben. Sexueller Kindesmissbrauch ist eine sexuelle Handlung, die einem Kind aufgezwungen wird, dem es an emotionaler und kognitiver Reife mangelt. Die Möglichkeit, ein Kind zu einer sexuellen Beziehung zu verführen, stützt sich auf die generelle Macht und Dominanz von erwachsenen oder älteren jugendlichen Täterlnnen, die sich deutlich vom Alter des Kindes, seiner Abhängigkeit und seiner untergeordneten Position abheben (Sgroi et al. 1982). Bei den Opfern handelt es sich um entwicklungsmäßig unreife Personen. Unreife kann sich auf emotionaler, psychischer und kognitiver Ebene äußern.

„Klinische Definition“: Sexueller Kindesmissbrauch ist jede sexuelle Handlung oder Erfahrung, die einem Kind aufgezwungen wird, welche ein emotionales, 
körperliches oder sexuelles Trauma zur Folge hat (Bagley und King 1990). Hier kritisiert Fegert aber die schwer nachzuweisende lineare Kausalität (Fegert, 1992). So konnte in einigen empirischen Studien gezeigt werden, dass sexueller Missbrauch nicht unbedingt mit negativen Folgeerscheinungen verbunden sein muss (Caffaro-Rouget, Lang und van Santen, 1989; Conte und Schuermann 1987). Bei der Verarbeitung bzw. Bewältigung des sexuellen Missbrauchs spielen zahlreiche andere Faktoren, wie familiäre Beziehungen, Partnerschaft, etc., eine bedeutende Rolle (Cole und Putnam 1992; Mullen et al. 1993).

Es wird deutlich, dass jede Definition unterschiedliche Kriterien heranzieht und in den Vordergrund stellt, wie z.B. die Art der sexuellen Handlung (Kontakt, Vorgehen, etc.), das Macht- und Autoritätsverhältnis, das Geschlecht des Opfers, die Folgen des Missbrauchs. In der vorliegenden Arbeit lehnen wir uns an die von Amann und Wipplinger (1997) aufgestellte Definition an, die aus unserer Sicht auf Grund ihrer Aufteilung in eine "enge" und „weite" Definition des sexuellen Missbrauchs die Aufgabenstellung in einer umfassenden, aber differenzierten Weise gut abdeckt. Erweitert wird die Definition von Amann und Wipplinger noch um die Erkenntnisse von McGee (1995), die in die von uns verwendete Definition integriert wurden.

Die „enge Definition“ ist meist Grundlage von empirischen Studien. Eine Vielzahl von Autoren haben sich der engen Definition angeschlossen, interpretieren diese aber teilweise wieder unterschiedlich. Die enge Definition beschreibt sexuellen Missbrauch vorwiegend als körperlichen Kontakt zwischen Täter und Opfer, wie oralen, analen und genitalen Geschlechtsverkehr. Bagley (1995) vertritt die Ansicht, dass sexueller Missbrauch möglichst eng definiert sein sollte, um sich auf Handlungen zu beschränken, die mit großer Wahrscheinlichkeit Schaden und psychische Beeinträchtigungen für junge Menschen verursachen. Sexueller Kindesmissbrauch ist zumindest ein körperlicher Kontakt mit dem unbekleideten Genitalbereich oder Brustbereich des Kindes (eingeschlossen ist ein Kontakt unter der Kleidung), der vom Kind oder dem Jugendlichen nicht gewollt ist. Ein 
Kind oder ein Jugendlicher wird definiert als jemand, der den 17. Geburtstag noch nicht erreicht hat (Bagley 1995). Nash postuliert, dass der sexuelle Kontakt sich zwischen einem Kind unter 17 Jahren und einem Erwachsenen, der mindestens um fünf Jahre älter ist, abspielen muss (Nash et al. 1993). Diese Forderung des Altersunterschied wird vielfach kritisiert. Laut Engfer (1997) sind Altersangaben, die eine "normale" sexuelle Beziehung von Täter-Opfer Beziehungen abgrenzen, nicht klar zu definieren. Sexuelle Übergriffe gehen nicht nur von Erwachsenen, sondern in bis zu 30\% - 45\% der Fälle von Jugendlichen oder wenig älteren Tätern aus (Bange 1992, Murphy and Peters 1992). Schäfer (2000) schließt sich Engfer an und betont, dass statt der Eingrenzung durch einen Altersunterschied, ein bestehendes Machtgefälle zwischen Täterln und Opfer als Kriterium heranzuziehen ist. Dieses Machtgefälle kann auch bei gleichaltrigen Jugendlichen vorhanden sein, was wiederum den Zwang zu ungewollten sexuellen Handlungen ermöglicht. Wichtig erscheint, dass keines der Opfer sich zum Missbrauchszeitpunkt in die Lage versetzt fühlt, eine eigene Entscheidung für oder wider fällen zu können. Dieser Ansicht wird auch in der vorliegenden Arbeit gefolgt und als Kriterium für sexuellen Missbrauch zu Grunde gelegt. Gemeinsames und wesentliches Kennzeichen aller Interpretationen zur „engen Definition“ ist, dass als sexueller Missbrauch nur Handlungen verstanden werden, die mit einem direkten Körperkontakt zwischen Täterln und Opfer verbunden sind.

Die „weite Definition“ unternimmt den Ansatz, das Phänomen des sexuellen Missbrauchs in seinem gesamten Umfang zu beschreiben. Es beinhaltet damit auch die durch die enge Definition erfassten Missbrauchsformen. Allerdings erweitert sie die Definition um jede Art der sexuellen Handlung, wie obszöne Anreden, Belästigung, Exhibitionismus, Anleitung zur Prostitution, die Herstellung von pornografischem Material usw. Die weite Definition berücksichtigt entsprechend auch „Nicht-Kontakthandlungen“ (Waytt und Peters 1986)

Amann und Wipplinger ist es mit dieser Unterteilung gelungen, eine höhere 
Genauigkeit bei der Definition des sexuellen Missbrauchs zu schaffen. McGee (1995) hat diese Definition noch erweitert, indem er - aufbauend auf Schweregraden des Missbrauchs - eine Dreiteilung in „leicht", „mittelschwer" und „schwer" vorschlägt: „Leichter sexueller Missbrauch“ entspricht der weiten Definition, „schwerer sexueller Missbrauch“ der engen Definition und „mittelschwerer sexueller Missbrauch“präzisiert den Bereich zwischen der weiten und engen Definition. Diese auch in der Arbeit zu Grunde gelegte Definition soll im folgenden erläutert werden:

Ein „leichter sexueller Missbrauch“ beschreibt Handlungen, die der sexuellen Erregung und Befriedigung des Täters dienen, aber keinerlei direkten körperlichen Kontakt mit sich bringen. Als „mittelschwerer sexueller Missbrauch“ werden Handlungen definiert, die der sexuellen Erregung und Befriedigung des Täters dienen und mit körperlichem Kontakt einhergehen, bei denen es aber nicht zum vollzogenen Geschlechtsakt mit Penetration kommt. Ein „schwerer sexueller Missbrauch“liegt bei oraler, vaginaler oder analer Penetration vor.

\subsubsection{Definition körperliche Misshandlung}

Als einer der ersten beschrieb der amerikanische Kinderarzt C. H. Kempe (Kempe et al. 1968) die Kindesmisshandlung unter dem Begriff des „batteredchild-syndrom“. Später unterschied Remschmidt (1986) die körperliche Kindesmisshandlung von der Vernachlässigung, der psychischen und sexuellen Misshandlung. Bei der körperlichen Misshandlung steht meistens zunächst der Züchtigungsversuch der Eltern gegenüber dem Kind im Vordergrund. Häufig können aber bei einer Eskalation schwerwiegende Verletzungen bis hin zum Todesfall die Folge sein. Martinius (1989) definierte körperliche Misshandlung als „ungesteuertes Strafen und ein weder inhaltlich, noch zeitlich isolierbares Ereignis, sondern ein Vorgang, innerhalb dessen die körperliche Misshandlung den zwar kennzeichnenden Schritt, aber eben doch nur einen Schritt darstellt. 
Dieser Schritt ist gewaltsam, herausragend und wegen der durch inn erzeugten körperlichen Schäden mehr oder weniger oft Anlass für seine Entdeckung“.

Nach Engfer (1997) fallen unter körperliche Misshandlung Schläge oder andere körperliche gewaltsame Handlungen (Stöße, Verbrennungen, Stiche u.s.w.), die beim Opfer zu Verletzungen führen können. Dabei stellt Engfer auch auf die Empfindlichkeit des Kindes entsprechend seines Alters ab. So kann ein heftiges Schütteln eines Säuglings oder Kleinkindes zu subduralen Hirn- und Glaskörperblutungen führen, während bei größeren Kindern meist keine körperlichen Verletzungen entstehen. Dornes (1997) betont zusätzlich in seiner Definition der körperlichen Misshandlung die Häufigkeit der körperlichen Bestrafung.

Das Bundesministerium für Familie, Senioren, Frauen und Jugend (2000) definiert eine körperliche Misshandlung als all jene Handlungen, vom einzelnen Schlag mit der Hand, über Prügeln, Festhalten und Würgen bis hin zum gewaltsamen Angriff mit Riemen, Stöcken, Küchengegenständen und Waffen, die zu einer nicht-zufälligen körperlichen Verletzung eines Kindes führen, wobei es vor allem zu Blutergüssen, Prellungen Schädel- und Knochenbrüchen, aber auch zu inneren Verletzungen und zu Verbrennungen kommt.

Um wieder eine Klassifizierung der Schweregrade der körperlichen Misshandlung zu ermöglichen, stützen wir uns auf die Arbeiten von McGee (1995): Als „leichte Misshandlung" definieren wir gelegentliche bis häufige Schläge, die keine körperlichen Schäden zur Folge hatten. Als „mittelschwere/mittlere Misshandlung" werden Kontakthandlungen definiert, in denen das Kind zwar gesundheitlich gefährdet war, es aber nicht zu körperlichen Schäden kam. Als "schwere Misshandlung" werden alle Handlungen eingestuft, die körperliche Folgeschäden wie Verbrennungen oder Knochenbrüche beim Kind nach sich ziehen. 


\subsubsection{Definition Vernachlässigung}

Unter Kindesmisshandlung wird allgemein eine gewaltsame psychische und physische Beeinträchtigung von Kindern durch Dritte - meistens der Eltern oder Erziehungsberechtigten - verstanden. Die Vernachlässigung zeichnet sich dabei durch eine „Unterlassung" aus. Kinder werden vernachlässigt, wenn sie von Eltern und Betreuungspersonen unzureichend ernährt, gepflegt, gefördert, gesundheitlich versorgt, beaufsichtigt und/oder vor Gefahren geschützt werden (Engfer 1997).

Ähnlich sieht Dornes den Tatbestand gegeben, wenn eine deutliche und dauerhafte Vernachlässigung der grundlegenden körperlichen und psychischen Bedürfnisse des Kindes nach Nahrung, Sauberkeit, bedarfsgerechter medizinischer Versorgung und affektiver Kommunikation vorliegt (Dornes 1997).

Zuravin (1991) unterteilt die Vernachlässigung in Subtypen, die auf verschiedenen Kategorien elterlichen Fehlverhaltens bzw. das des Erziehungsberechtigten basieren. Hierzu gehören unzureichende oder fehlende medizinische Versorgung, verzögerte medizinische Versorgung, Vernachlässigung der Aufsichtspflicht und Ignorierung von Gefahrenquellen.

Mit der Klassifizierung der Schweregrade der Vernachlässigung nach McGee (1995) ergibt sich für unsere Definition folgendes Bild: Eine "leichte Vernachlässigung" wird aufgefasst als Fehler der Eltern, welche schmerzhaft für das Kind sein können. „Mittelschwere Vernachlässigung“ bezieht sich auf Unterlassungen, die das Kind in die Gefahr von Entwicklungsabweichungen bringen und betreffen elterliche Festigkeit und Verfügbarkeit. „Schwere Vernachlässigung" umfasst elterliche Unterlassungen, die das Kind der Gefahr körperlicher Schädigung aussetzen. 


\subsubsection{Definition familiäre Gewalt}

Nach McGee et al. (1995) liegt der Tatbestand von familiärer Gewalt vor, wenn Kinder der physischen Gewalt zwischen Eltern und/oder Gewalt zwischen Eltern und ihren Partnern ausgesetzt sind. Die familiäre Gewalt wird bei uns in Anlehnung an McGee in drei Skalen abhängig von dem Grad der verwendeten Gewalt quantifiziert:

Als "leichte familiäre Gewalt" werden kontaktlose Erfahrungen zwischen den Eltern eingeordnet. Eine "mittelschwere" Konfrontation mit familiärer Gewalt ist definiert als Erleben waffenloser, körperlicher Auseinandersetzungen zwischen den Eltern, die allerdings keine schweren Verletzungen zur Folge haben. "Schwere" Konfrontation mit familiärer Gewalt liegt bei einem Miterleben von Auseinandersetzungen der Eltern mit massiver körperlicher Bedrohung einer Person.

Erleben Kinder die physische Gewalt zwischen den Eltern bzw. Erziehungsberechtigten mit, so kann auch dies zu einer Traumatisierung der Kinder führen. Häufig ist Gewalt zwischen Eltern bzw. Erziehungsberechtigten in Familien mit Suchterkrankungen zu finden.

\subsubsection{Definition emotionale Misshandlung}

Jede Art einer Misshandlung beinhaltet in ihren Grundzügen eine emotionale Misshandlung. Dies gilt sowohl für die im allgemeinen Sinne verstandene psychische emotionale Misshandlung ohne körperliche Übergriffe, als auch für jede Art der körperlichen Misshandlung, da diese immer auch eine seelische Komponente enthält.

McGee und Wolfe haben 1991 einen ersten Ansatz zur Systematisierung der emotionalen Misshandlung unternommen. Sie unterteilen dazu die emotionale 
Misshandlung in vier Kategorien. Die dazu herangezogenen Parameter sind zum einen die physische oder nicht-physische/psychische Misshandlungsform seitens der Eltern. Zum anderen stellen sie auf die Auswirkungen auf das Kind ab, die ebenfalls physisch oder nicht-physische/psychische Folgen umfassen können.

Im allgemeinen wird unter emotionalem Missbrauch die Kategorie der psychischen Misshandlung mit psychischen Folgen für das Kind verstanden. So liegt nach Dornes ein emotionaler Missbrauch bei ständigem Kritisieren des Kindes, Drohen, Verächtlichmachen, Einsperren in einen dunklen Raum, etc. vor (Dornes 1997).

Engfer (1997) verwendet statt emotionaler Misshandlung den Begriff der psychischen Misshandlung. Er umfast alle Handlungen und Unterlassungen von Eltern oder Bezugspersonen, die Kinder ängstigen, überfordern, innen das Gefühl der Wertlosigkeit vermitteln und sie in ihrer psychischen und/oder körperlichen Entwicklung beeinträchtigen können. Engfers Definition der psychischen Misshandlung gliedert sich somit in jenen Teil der Definition von McGee und Wolfe (1991) ein, der auf psychische Misshandlungsformen mit psychischen Auswirkungen abstellt. Engfer weist aber auch auf die Schwierigkeiten bei der Definition und Erkennung der emotionalen Misshandlung hin. So zählen nicht nur sadistische Formen der seelischen Grausamkeit zur emotionalen Misshandlung, sondern auch Varianten elterlichen Verhaltens wie z.B. die demonstrative Bevorzugung eines Geschwisterkindes, Kinder einzuschüchtern, sie häufig zu beschimpfen, zu isolieren oder sie mit langanhaltendem Liebesentzug zu bestrafen. Die Schwierigkeit liegt nun darin, dass die Grenze zwischen üblichen und weitgehend tolerierten Praktiken (z.B. Bestrafen mit Hausarrest, Liebesentzug) und psychisch schädigendem Elternverhalten schwer zu ziehen ist.

Das Bundesministerium für Familie, Senioren, Frauen und Jugend (2000) definiert die emotionale Misshandlung folgendermaßen: „Sie umfasst qualitativ 
und quantitativ ungeeignete und unzureichende, altersinadäquate Handlungen und Beziehungsformen und -verhältnisse von Sorgeberechtigten zu Kindern in der Form der Ablehnung, des Überforderns, des Herabsetzens, des Ängstigens und Terrorisierens, des Isolierens, des Korrumpierens, der Ausbeutung und der Verweigerung emotionaler Zuwendung und Unterstützung, wodurch das Bestreben eines Kindes, seine affektiven, kognitiven und moralischen Entwicklungsbedürfnisse zu befriedigen, in einem Maße eingeschränkt, frustriert und durchkreuzt wird, dass die Persönlichkeitsentwicklung eines Kindes beeinträchtigt und geschädigt wird."

Analog zu den Ausführungen der anderen Missbrauchsformen, definieren wir emotionalen Missbrauches unter Berücksichtigung des Schweregrads (vgl. McGee 1995): "Leichte emotionale Misshandlung" bezieht sich dann auf indirekte negative Kommunikation, die Fehler in der elterlichen Rolle repräsentieren, z.B. schweigender Umgang mit dem Kind oder Verunglimpfung anderer, die das Kind gerne mag. „Mittelschwere emotionale Misshandlung“ umfasst indirekte, negative Kommunikation bezüglich des Wertes des Kindes, z.B. Beschuldigungen aussprechen oder Verspotten des Kindes. Als „schwere emotionale Misshandlung“ werden solche Taten der Eltern definiert, die einen direkten Angriff auf des Kindes Selbstverständnis oder Sicherheit darstellen, z.B. dem Kind sagen, dass es ungewollt ist, Drohung das Kind zu verlassen oder zu töten, oder Anwendung von extremer und demütigender Bestrafung.

\subsubsection{Definition Sucht}

Unter dem Begriff der Suchtstörungen werden alle Phänomene zusammengefasst, die mit der unkontrollierten, selbstschädigenden Einnahme psychotroper Substanzen und/oder dem ebenso unkontrollierten, selbstschädigenden Ausführen bestimmter Verhaltensweisen zusammenhängen. Im engeren Sinne, wie er in der Psychiatrie und klinischen Psychologie üblich ist, werden darunter die substanzbezogenen Störungen verstanden. Diese sind der 
Missbrauch psychotroper Substanzen, wie z.B. Alkohol, Opiate, Kokain usw., oder die Abhängigkeit von diesen Substanzen.

1873 informierte der Berliner Arzt Eduard Levinstein die Öffentlichkeit erstmals über eine Morphiumsucht. 1952 unternahm die WHO erstmals den Ansatz eine international verbindliche Definition der Sucht aufzustellen. Auf Grund der Heterogenität der Drogen erkannte die WHO, dass der Begriff der Sucht keine ausreichende Beschreibung bot, und führte 1964 den Rahmenbegriff der „Drogenabhängigkeit“ ein. Drogenabhängigkeit war „ein Zustand, der sich aus der wiederholten Einnahme einer Droge ergibt, wobei die Einnahme periodisch oder kontinuierlich erfolgen kann. Ihre Charakteristika variieren in Abhängigkeit von der benutzten Substanz" (WHO 1964). Gleichwohl wurden im allgemeinen Sprachgebrauch die Begriffe Sucht und Abhängigkeit synonym verwendet. Eine weitere Präzisierung der Begrifflichkeit der Sucht erfolgte 1981 durch die WHO. Sie ergänzte die Kategorien „nichtmedizinischer Gebrauch (abuse)" und „fehlerhafte ärztliche Anwendung (misuse)" durch die Kategorien „unerlaubter Gebrauch (unsanctioned use)“, „gefährlicher Gebrauch (hazardous use)“, „dysfunktionaler Gebrauch (dysfunctional use)“ und „schädlicher Gebrauch (harmful use)“. 1985 formulierte die WHO die Sucht folgendermaßen: „Sucht (addiction) ist ein Zustand periodischer oder chronischer Intoxikation, der durch die wiederholte Einnahme einer (natürlichen oder synthetischen) Droge hervorgerufen wird. Ihre Charakteristika sind ein überwältigendes Verlangen oder Bedürfnis (zwanghafter Art), die Drogeneinnahme fortzusetzen und sich diese mit allen Mitteln zu verschaffen, eine Tendenz zur Dosissteigerung, eine psychische (psychologische) und allgemein eine physische Abhängigkeit von den Drogenwirkungen, zerstörerische Wirkung auf das Individuum und die Gesellschaft“ (WHO 1985). Mit der Veröffentlichung des Kapitel V (F) der ICD-10 ergänzte die WHO 1993 den deskriptiven Ansatz, so dass jetzt einzelne Substanzen und distinkte klinische Syndrome getrennt betrachtet werden können (Dilling et al. 1993, 1994). 
Die Entwicklungen des Verständnisses der Sucht haben auch die Untersuchungen zu den Zusammenhängen zwischen psychischen Störungen, stofflichem Missbrauch und Realtraumatisierungen gelenkt. Gerade der deskriptive Ansatz hilft bei der differenzierten Untersuchung und erlaubt differentielle therapeutische Ansätze (vgl. Krausz und Müller-Thompson 1994). 


\section{Methoden und Ergebnisse}

\subsection{Methoden}

\subsubsection{Auswahl der Stichprobe}

Die notwendigen Befragungen wurden im Rahmen dieser Studie in den Suchtstationen der psychiatrischen Kliniken in München, Augsburg, Aachen und Lübeck sowie der Drogenambulanz des Schwabinger Krankenhauses München im Jahre 2000 und 2001 durchgeführt. Insgesamt wurden 100 Patienten mit einer Abhängigkeit von illegalen Drogen befragt, wobei nach dem Rücklauf die Unterlagen von 83 PatientInnen auswertbar waren. Außerdem erfolgte die Befragung von 100 Patienten mit einer Alkoholabhängigkeit, zu denen sich die Ergebnisse in einer parallel durchgeführten Dissertation finden.

Zur Erhebung der Daten kamen zwei Fragebögen und ein Interview zum Einsatz: Der European Addiction Severity Index (EuropASI) ist als Interview aufgebaut und erfasst wichtige Informationen über jene Lebensbereiche des Patienten, die in einem Zusammenhang mit der Substanzabhängigkeit stehen. Der Fragebogen der Symptom-Checkliste SCL 90-R nach Derogatis (1977) und Franke (1995) dient zur Erfassung der psychischen Symptombelastung. Weiterhin verwendeten wir einen selbsterstellten Traumafragebogen in Anlehnung an McGee et al. (1995), der eine Einteilung in Schweregrade des Missbrauchs ermöglicht.

Mit jedem Patienten wurde persönlich und auf freiwilliger Basis eine Einzelbefragung durchgeführt. Die Patienten wurden ausführlich über Inhalte und Nutzen der Untersuchung aufgeklärt und unterschrieben eine Einverständniserklärung. Anschließend wurde das Interview (EuropASI) durchgeführt. Nach Möglichkeit hat der Patient direkt im Anschluss die SymptomCheckliste SCL 90-R und den Traumafragebogen ausgefüllt. Alle Daten wurden 
streng anonym behandelt. Die Fragebögen wurden mit laufenden Codenummern versehen.

\subsubsection{Beschreibung des Interviews "European Addiction Severity Index" (EuropASI)}

Der European Addiction Severity Index (EuropASI) ist ein semi-standardisiertes Interview, mit dem wichtige Informationen über jene Lebensbereiche des Patienten erfasst werden, die in einem Zusammenhang mit der Substanzabhängigkeit stehen. Ziel ist es, eine Einschätzung des Zustandes des Patienten bezüglich der verschiedenen Lebensbereiche zu gewinnen. Die Interview-Daten bilden sowohl für die Forschung, als auch für die klinische Praxis die Grundlage für die Erstellung eines Profils über die Problemausprägung des Patienten.

Der Addiction Severity Index (ASI) wurde 1980 in den USA erstmalig eingeführt. Als Grundlage für die deutsche Version des EuropASi diente das amerikanische Original von T. McLellan in seiner 5. Auflage (1992) und die europäische Version EuropASI von A. Kokkevi, Ch. Hartgers, P. Blanken, E.-M. Fahrner, G. Pozzi, E. Tempesta, und A. Uchtenhagen (1993). Die deutsche Übersetzung des European Addiction Severity Index entstand durch die Zusammenarbeit von B. Gsellhofer, H. Küfner, M. Vogt und D. Weiler (1994). Eine Auswertung des Fragebogens erfolgte mittels des „vorläufigen Verfahrens für die Berechnung der EuropASI Composite Scores“ nach Koeter \& Hartgers (1997).

Der EuropASI setzt sich aus 150 Items zusammen, die wiederum sieben inhaltlichen Problembereichen (Subskalen) zugeordnet sind. In jedem Problembereich werden Fragen zu den Problemen der Patienten gestellt, eine Selbsteinschätzung des Patienten getroffen, eine Einschätzung des Interviewers zum Interventionsbedarf und zur Beurteilung der Zuverlässigkeit der Patientenangaben vorgenommen sowie die Möglichkeit zu Kommentaren durch den Interviewer gegeben. Fragen zu Anzahl, Dauer und Ausmaß der 
Schwierigkeiten beziehen sich entweder auf die gesamte Lebensdauer oder auf das Zeitfenster innerhalb der letzten 30 Tage vor der stationären Aufnahme bzw. der aktuellen Behandlung. Die Selbsteinschätzungen zur Problembelastung bzw. zum Beratungs- oder Behandlungsbedarf werden auf Basis einer Skala von 0 (gar kein Problem) bis 4 (extremes Problem) abgegeben. Die Auswertung mittels der "Composite Scores" verwertet und gewichtet die Antworten zu den Items des jeweiligen Problembereichs und berechnet daraus einen Index für den jeweiligen Problembereich, der die Problemausprägung des Patienten wiederspiegelt. Ein Interview dauert circa 45 bis 60 Minuten.

Als kurzer Überblick dient die folgende Beschreibung der Fragen des EuropASI, gegliedert nach einzelnen Problembereichen: In dem Problembereich „Allgemeine Angaben“ werden im wesentlichen demografische Daten wie Alter, Geschlecht, Wohnort, Dauer der aktuellen Adresse, Staatsangehörigkeit, Geburtsland der Eltern sowie Aufenthalt in einer institutionellen Umgebung und gegebenenfalls die Dauer des Aufenthalts erhoben. In dem Problembereich „Körperlicher Zustand“ interessieren chronische Erkrankungen, Anzahl der Krankenhausaufenthalte, Hepatitis-Infektionen, HIV-Erkrankung, verschriebene Medikamente, Arztbesuch sowie die Anzahl der Tage im den letzten 30 Tagen mit gesundheitlichen Problemen. Die Fragen zur „Arbeits- und Unterhaltssituation" gehen auf Schulbildung, Berufsausbildung, Erwerbstätigkeit und letztes Beschäftigungsverhältnis ein. Es interessieren weiterhin die längste Arbeitslosigkeit, Schulden, die Quelle des Lebensunterhaltes sowie berufliche Probleme in den letzten 30 Tagen. Bei „Drogen- und Alkoholgebrauch“ wird nach dem Alter beim ersten Drogen- oder Alkoholkonsum, dem Alter bzw. den Jahren des regelmäßigen Konsums, der Art und Weise der Einnahme, dem Gebrauch bzw. der Einnahme anderer Suchtstoffe, den Abstinenzperioden, der Anzahl der Delirien und den Vorbehandlungen gefragt. Detailliert wird auf die Frage nach der Art der Droge, der Behandlung und auch auf den Gebrauch in den letzten 30 Tagen eingegangen. In dem Problembereich "Rechtliche Probleme“ wird der Patient nach Führerscheinentzug, Verkehrsdelikten mit und ohne Alkohol, 
sonstigen Delikten und Verurteilungen gefragt. Der Schwerpunkt liegt hierbei allerdings auf Straftaten, die sich nicht auf den Straßenverkehr beziehen. Der Problembereich „Familie und Sozialbeziehung" beleuchtet die eigenständigen, singulären Beziehungsprobleme des Patienten unter Zurückstellung der durch die Sucht induzierten Probleme. Dazu beschreibt der Patient seine Familien- und Wohnsituation, seine Freizeitaktivitäten, seine Beziehungssituation zu Partnern und Freunden sowie seine Zufriedenheit bzw. Probleme. Ergänzt wird dieser Bereich durch die Fragen zum "familiären Hintergrund", der die psychischen oder die Alkohol- und Drogenprobleme der Angehörigen des Patienten zusammenfasst. In dem Problembereich „Psychischer Status" stehen die Fragen nach den stationären bzw. ambulanten Behandlungen, nach einer Rente wegen psychischer Probleme, nach belastenden Ereignissen im letzten halben Jahr und nach Zeiträumen, in denen bestimmte psychische Probleme aufgetreten sind. Es werden psychische Probleme, die nicht als Resultat des Alkohol- oder Drogenmissbrauchs zu verstehen sind, sowie Depressionen, Ängste, Halluzinationen, Konzentrationsstörungen, gewalttätiges Verhalten, Medikamente für psychische Probleme, Suizidgedanken und-versuche, einzeln aufgeführt.

Ein Schweregrad-Rating des Interviewers bezüglich des Ausmaßes der jeweiligen Probleme, d.h. der Grad, in dem der Proband nach Einschätzung des Interviewers einer Behandlung bedarf, wird am Ende jedes Problembereichs registriert, zusammen mit einem Zuverlässigkeits-Rating, d.h. inwieweit eine falsche Selbstdarstellung des Patienten vorliegen könnte.

Die Auswertung des EuropASI-Interviews erfolgt mittels eines vorläufigen Verfahrens der Berechnung von gewichteten Summenscores (sog. Composite Scores) nach Koeter und Hartgers. Dem statistischen Prozedere von McLellan et al. (1985) folgend, werden sogenannte Subskalen konstruiert, die den Untertests bzw. Problembereichen des EuropASI-Interviews entsprechen. Im folgenden werden die Bezeichnungen Subskala und Untertest sowie Summenmesswert und Composite Score synonym verwendet. 
Zur Bestimmung des Composite Scores wird ein Score für jeden Problembereich separat errechnet. Die Composite Scores geben den Bedarf nach weiterer Behandlung an. Aus der Berechnung nach Maßgabe des Composite Scores ergeben sich Zahlenwerte zwischen 0 (kein Problem) und 1 (schwerwiegendes Problem). Für diese Scores werden nur diejenigen Fragen zur Berechnung herangezogen, die sich auf die letzten 30 Tage beziehen oder aber auf die aktuelle Situation Bezug nehmen. Deshalb sind sie für eine Erfassung der Veränderung des Patientenzustandes und der Behandlungseffektivität in Verlaufsstudien geeignet (McLellan et al., 1992). Für die Klassifizierung der Ergebnisse orientieren wir uns an der Einteilung nach McLellan:

- bis 0,44: Probleme geringen Ausmaßes

- 0,45 bis 0,74: Probleme mäßigen Ausmaßes

- 0,75 bis 1,00: Probleme schweren Ausmaßes

\subsubsection{Beschreibung des Fragebogens „Symptom Checkliste“ (SCL 90-R)}

Die Symptom-Checkliste SCL 90-R (Derogatis 1977, Derogatis \& Savitz 1999, Franke 1995) gehört weltweit zu den in Klinik und Forschung am häufigsten eingesetzten Verfahren zur Erfassung der psychischen Symptombelastung (psychological distress). Sie ist ein Messinstrument zur Erfassung der vom Patienten selbstbeurteilten psychischen und körperlichen Belastung. Die SCL 90$\mathrm{R}$ wird vom Patienten selbstständig ausgefüllt. Dazu gibt der Patient auf einer fünfstufigen Skala an, wie häufig er in den vergangenen sieben Tagen unter dem mit dem Item erfassten Symptom gelitten hat. Beurteilt werden die Items in den Ausprägungen (mit den entsprechenden Itemkennwerten) „überhaupt nicht“ (0), „ein wenig“ (1), „Ziemlich“ (2), „stark“ (3) und „sehr stark“ (4). Das Ergebnis gliedert sich in neun Subskalen (Somatisierung, Zwanghaftigkeit, Unsicherheit im Sozialkontakt, Depressivität, Ängstlichkeit, Aggressivität/Feindseligkeit, phobische Angst, paranoides Denken, Psychotizismus) und drei Globale 
Kennwerte (GSI - global severity index, PSDI - positive symptom distress index, PST - positive symptom total). Sieben Items gehen als Zusatzinformationen in die Berechnung der drei Globalen Kennwerte mit ein.

Im folgenden soll eine Kurzbeschreibung der abgebildeten Skalen gegeben werden. Zuerst wird auf die neun Kennwerte eingegangen, anschließend werden die drei Globalen Kennwerte erläutert: Die Subskala "Somatisierung“ erfasst über 12 Items einfache körperliche Belastungen bis hin zu funktionellen Störungen. Die Anzeichen und Symptome haben eine hohe Prävalenz bei Störungen funktioneller Ätiologie. Bei „Zwanghaftigkeit“ beschreiben zehn Items leichte Konzentrations- und Arbeitsstörungen bis hin zur ausgeprägten Zwanghaftigkeit: Gedanken, Impulse und Handlungen, die als konstant vorhanden und nicht änderbar vom Patienten erlebt und gleichzeitig als ich-fremd und ungewollt empfunden werden. Die Subskala „Unsicherheit im Sozialkontakt“ bezieht sich mit neun Items auf Gefühle persönlicher Unzulänglichkeit und Minderwertigkeit. Selbstabwertung, deutliche Gefühle des Unwohlseins, der akuten Unsicherheit und negative Erwartungen in interpersoneller Kommunikation sind charakteristische Manifestationen. Unter "Depressivität" werden 13 Items zur Erfassung von Traurigkeit bis hin zur schweren Depression einbezogen. Symptome dysphorischer Stimmung zeigen gesunkenes Interesse am allgemeinen Leben, verringerte Motivation und den Verlust vitaler Energien; zusätzlich finden sich Gefühle von Hoffnungslosigkeit, Suizidgedanken und andere somatische und kognitive Korrelate der Depression. Die Subskala „Ängstlichkeit" erfasst über 10 Items körperlich spürbare Nervosität bis hin zu tiefer Angst. Die Skala fokussiert auf manifestierte Angst mit Nervosität, Spannung und Zittern, Panikattacken und Schreckgefühlen. Die Subskala „Aggressivität und Feindseligkeit“ beinhaltet unter Einbeziehung von sechs Items Reizbarkeit und Unausgeglichenheit bis hin zu starker Aggressivität mit feindseligen Aspekten. Die Skala bezieht sich auf Gedanken, Gefühle und Handlungen, die charakteristisch für den negativen Gefühlszustand von Ärger sind und Aggression, Irritierbarkeit, Zorn und Verstimmung erfassen. „Phobische 
Angst" wird mittels sieben Items in der Ausprägung eines leichtes Gefühls von Bedrohung bis hin zu massiver phobischer Angst umrissen: Andauernde und unangemessene Furcht als Reaktion auf bestimmte Personen, einen Platz, ein Objekt oder eine charakteristische Situation, die zu Vermeidungs- oder Fluchtverhalten führt. In der Subskala „Paranoides Denken“ geben sechs Items Misstrauen und Minderwertigkeitsgefühle bis hin zu starkem paranoiden Denken an. Als primäre Aspekte der Störung werden Gedankenprojektion, Feindseligkeit, Argwohn, Einengung, Angst vor Autonomieverlust und wahnhafte Täuschungen verstanden. In der Subskala „Psychotizismus“ beschreiben zehn Items das milde Gefühl der Isolation und Entfremdung bis hin zur deutlichen Evidenz psychotischer Episoden. Die Skala umfasst eine kontinuierliche Dimension des menschlichen Erlebens, die von verzerrtem, isoliertem, schizoidem Lebensstil bis hin zu Primärsymptomen der Schizophrenie wie z.B. Gedankenzerfall oder Halluzinationen reichen.

Zusätzlich zu den Kennwerten der Subskalen geben drei Globale Kennwerte, der GSI (global severity index), der PSDI (positive symptom distress index) und der PST (positive symptom total) Auskunft über das Antwortverhalten über alle Items. Der „GS/" misst die grundsätzliche psychische Belastung. Die grundsätzliche psychische Belastung errechnet sich aus der Summe aller Antwortwerte dividiert durch die Zahl der beantworteten Items. Der „PSD" misst die Intensität der Antworten. Er berechnet sich aus dem Quotienten der Gesamtsumme der Itemwerte und der Anzahl belastender Symptome. Der „PST“ gibt Auskunft über die Anzahl der Symptome, bei denen eine Belastung vorliegt. Die Summe der Belastungstendenzen entspricht der Anzahl belastender Symptome. Damit ist die Anzahl der Items ausschlaggebend, die mit Antwortscores von 1 bis 4 (nicht 0 ) durch die Patienten beurteilt wurden. 


\subsubsection{Beschreibung des Münchener Traumafragebogens}

Der Münchener Traumafragebogen (im Folgenden als Traumafragebogen bezeichnet) erfasst Missbrauchserfahrungen im Kindes- und Jugendalter mit Berücksichtigung einer Schweregradeinteilung des Missbrauchs und wurde im Rahmen dieser Doktorarbeit in Anlehnung an McGee et al. (1995) erarbeitet. Da kein geeigneter validierter Fragebogen bezüglich der retrospektiven Erfassung von Missbrauchserfahrungen im Kindes- und Jugendalter vorlag, war dieser Schritt erforderlich. Dies geschah in enger Anlehnung an die in der Literatur vorgegebenen Definitionen. Insbesondere wurde auf die Arbeiten von McGee zurückgegriffen, um den Anforderungen der Objektivität, Reliabilität und Validität weitmöglich nachzukommen. Allerdings können erst fortführende Untersuchungen mit dem hier vorgestellten Traumafragebogen, insbesondere an Referenzgruppen, die Objektivität, Reliabilität und Validität endgültig nachweisen.

Der "Traumafragebogen“ (vgl. Anhang B) gliedert sich in zwei Teile. Der erste Teil geht auf die Person und die familiäre Situation des Patienten ein. Es werden Fragen zum derzeitigen Alter, dem Geschlecht, der Staatsangehörigkeit und zum Geburtsland gestellt. Um die familiären Umstände des Patienten zu beleuchten, sollen die Befragten Angaben zur Berufsgruppe und dem Einkommen der Eltern, der Anzahl der Geschwister und deren Geschlecht machen. In Anlehnung an die in der Literatur häufig zu findende Frage nach Trennungen von Bezugspersonen wird auch untersucht, ob vor bzw. nach dem sechsten Lebensjahr eine längere Trennung von den Eltern stattfand. Der zweite Teil des Fragebogens untersucht die möglichen Missbrauchserfahrungen der Patienten in der Kindheit. Er ist in fünf weitere Unterkapitel gegliedert, die auf die Fragen zu sexuellem Missbrauch, körperlicher Misshandlung, emotionaler Misshandlung, Vernachlässigung und Gewalt in der Familie eingehen. Dabei wird der Patient nach seiner subjektiven Einschätzung der Schwere des Missbrauchs gefragt. Er bestimmt den Schwererad auf einer Skala von 1 bis 10 ( $1=$ leicht, 10= schwer). Ferner wird der Patient gebeten, Angaben zu den Umständen des Missbrauchs zu machen. Als 
Übersicht sollen nun die Bereiche und der Aufbau des Traumafragebogen stichpunktartig aufgeführt werden:

- Fragen zur „Person und zur familiären Situation in der Kindheit“

- demographische Daten des Befragten

- berufliche Situation der Eltern

- Anzahl der Geschwister

- Trennung von den Eltern

- Fragen zu „sexueller Missbrauchserfahrung“ (Unterteilung in drei Schweregrade)

- Misshandlungen vor dem 14. Lebensjahr

- Selbsteinschätzung des Schweregrads

- Alter zum Zeitpunkt der ersten Missbrauchserfahrung

- Täterbeschreibung (Personenkreis, Alter, Gewaltanwendung)

- Häufigkeit

- Einschätzung des Befragten, ob Einfluss auf die heutige Sucht vermutet wird

- Fragen zu „körperlicher Misshandlungserfahrung“ (Unterteilung in drei Schweregrade)

- Misshandlungen vor dem 14. Lebensjahr

- Selbsteinschätzung des Schweregrads

- Alter zum Zeitpunkt der ersten Misshandlungserfahrung

- Täterbeschreibung (Personenkreis, Alter, Gewaltanwendung)

- Häufigkeit

- Einschätzung des Befragten, ob Einfluss auf die heutige Sucht vermutet wird

- Fragen zu „Vernachlässigung“(Unterteilung in drei Schweregrade)

- Selbsteinschätzung des Schweregrads

- Einschätzung des Befragten, ob Einfluss auf die heutige Sucht vermutet wird

- Fragen zu „Gewalt in der Familie“(Unterteilung in drei Schweregrade) 
- Selbsteinschätzung des Schweregrads

- Einschätzung des Befragten, ob Einfluss auf die heutige Sucht vermutet wird

- Fragen zu „emotionaler Misshandlungserfahrung“ (Unterteilung in drei Schweregrade)

- Selbsteinschätzung des Schweregrads

- Einschätzung des Befragten, ob Einfluss auf die heutige Sucht vermutet wird

Bei jeder Missbrauchsart werden leichte, mittlere und schwere Formen der Misshandlung unterschieden. Der Patient kann anhand der genannten Beispiele bestimmen, ob eine der Missbrauchsdefinitionen auf inn zutrifft.

\subsubsection{Datenverarbeitung und statistische Auswertung}

Die Grunddaten der Fragebögen wurden in einer Datei für das Programmpaket SPSS Version 11.0 für Windows erfasst. Mit SPSS wurde auch die statistische Auswertung durchgeführt. Für die Textverarbeitung, Tabellen und Grafiken kam Microsoft Word 2000 zum Einsatz.

Für die statistische Auswertung wurde eine Irrtumswahrscheinlichkeit von „p $<$ 0,05 “ als signifikant zu Grunde gelegt, eine Irrtumswahrscheinlichkeit von „p $<$ 0,001 " als hochsignifikant. Für die Auswertung wurden die Lage und Streuungsmaße berechnet, namentlich das arithmetische Mittel, Median, Standardabweichung, Maximum und Minimum.

U.a. wurden folgende verteilungsfreie Verfahren durchgeführt:

- Mann-Whitney U-Test mit Angabe der Irrtumswahrscheinlichkeit ( $p$ ), den mittleren Rängen und dem z-Wert

- Chi-Quadrat Test nach Pearson für nominalskalierte Daten mit Angabe des Chi-Quadrats $\left(\chi^{2}\right)$, der Zahl der Freiheitsgrade (df) und der 
Irrtumswahrscheinlichkeit (p)

- Kruskall-Wallis Test mit Angabe des mittleren Ranges, der Zahl der Freiheitsgrade (df), des Chi-Wertes $(\chi)$ und der Irrtumswahrscheinlichkeit (p) 


\subsection{Ergebnisse}

Die Ergebnisse aus der Studie werden in folgender Reihenfolge dargestellt: Zunächst wird die soziodemografische Charakterisierung der Stichprobe vorgestellt. Es folgen die Ergebnisse zu den Auswirkungen von allgemeinem Missbrauchs, sexuellem Missbrauch, körperlicher Misshandlung, Vernachlässigung, familiärer Gewalt und emotionaler Misshandlung, wobei jeweils die Problembereiche nach EuropASI, die psychische Symptombelastung nach SCL 90-R sowie mögliche geschlechtsspezifische Unterschiede beschrieben sind. Anschließend soll auf die, mit dem Traumafragebogen ermittelte, Unterscheidung der Schweregrade bei den Missbrauchs- bzw. Misshandlungsarten und deren Auswirkungen eingegangen werden. Schließlich beziffern wir noch die absolute Belastung der Missbrauchsgruppen.

\subsubsection{Soziodemographische Ergebnisse zur Stichprobe}

In die vorliegende Untersuchung konnte von insgesamt 100 befragten drogenabhängigen Patienten der Rücklauf von 83 Patienten verwendet werden. Diese setzten sich zusammen aus 28 Frauen (33,7\%) und 55 Männern (66,3\%). Die Lage- und Streuungsmaße für das Alter sind getrennt nach Geschlecht in der folgenden Tabelle dargestellt. Der Altersunterschied zwischen Männern und Frauen wurde hierbei nicht signifikant $(p=0,057)$.

Tabelle 1: Stichprobe nach Alter und Geschlecht

\begin{tabular}{llll}
\hline Alter nach Geschlecht & Männer & Frauen & Gesamt \\
\hline Mittelwert & 31,3 & 28,4 & 30,3 \\
\hline Standardabweichung & 6,5 & 6,4 & 6,6 \\
\hline Minimum & 19 & 19 & 19 \\
\hline Maximum & 48 & 42 & 48 \\
\hline
\end{tabular}


Weitere soziodemographische Merkmale zur Wohnortgröße, Staatsangehörigkeit, zum höchsten erreichten Schulabschluss und zur Frage, ob eine abgeschlossene Berufsausbildung erreicht wurde, sind in Tabelle 2 zusammenfassend dargestellt.

Tabelle 2: Stichprobe nach Wohnortgröße, Nationalität, Schulabschluss und Berufsausbildung

\begin{tabular}{|c|c|c|c|c|}
\hline & & Männer (\%) & Frauen (\%) & Gesamt (\%) \\
\hline \multirow[b]{2}{*}{ Größe des } & Großstadt & $46(83,6)$ & $24(85,7)$ & $70(84,3)$ \\
\hline & mittelgroße Stadt & $7(12,7)$ & $3(10,7)$ & $10(12,1)$ \\
\hline \multirow[t]{2}{*}{ Wohnorts } & Kleinstadt & $2(3,6)$ & 0 & $2(2,4)$ \\
\hline & k.A. & 0 & $1(3,6)$ & $1(1,2)$ \\
\hline \multirow{3}{*}{ Nationalität } & Deutschland & $46(83,6)$ & $25(89,3)$ & $71(85,5)$ \\
\hline & EU-Länder & $2(3,6)$ & $1(3,6)$ & $3(3,6)$ \\
\hline & andere & $7(12,7)$ & $2(7,1)$ & $9(10,9)$ \\
\hline \multirow{5}{*}{ Schulabschluss } & Hauptschule & $26(47,3)$ & $11(39,3)$ & $37(44,6)$ \\
\hline & Mittlere Reife & $16(29,1)$ & $12(42,9)$ & $28(33,7)$ \\
\hline & Abitur & $3(5,5)$ & $1(3,6)$ & $4(4,8)$ \\
\hline & Fachschule/Akademie & $3(5,5)$ & 0 & $3(3,6)$ \\
\hline & ohne Abschluss & $7(12,7)$ & $4(14,3)$ & $11(13,3)$ \\
\hline \multirow{3}{*}{$\begin{array}{l}\text { Berufsaus- } \\
\text { bildung }\end{array}$} & ja & $30(54,5)$ & $13(46,4)$ & $43(51,8)$ \\
\hline & nein & $24(43,6)$ & $15(53,6)$ & $39(47,0)$ \\
\hline & k.A. & $1(1,8)$ & 0 & $1(1,2)$ \\
\hline
\end{tabular}

Der mit über $80 \%$ überwiegende Teil der Studienpopulation wohnte demnach zum Erhebungszeitpunkt in der Großstadt, ist deutscher Staatsangehöriger und hatte einen Hauptschul- bzw. Realschulabschluss. 13\% der Befragten verfügten über keinen Schulabschluss. Fast die Hälfte der Patienten $(47,0 \%)$ konnte keine abgeschlossene Berufsausbildung vorweisen.

Im Folgenden werden verschiedene Merkmale des Lebenskontextes der Patienten dargestellt. Tabelle 3 gibt einen Überblick über Familienstand und die aktuelle Wohnsituation. 
Tabelle 3: Familienstand und Wohnsituation

\begin{tabular}{lllll}
\hline & & Männer (\%) & Frauen (\%) & Gesamt (\%) \\
\hline \multirow{4}{*}{ Familienstand } & verheiratet & $5(9,1)$ & $4(14,3)$ & $9(10,8)$ \\
\cline { 2 - 5 } & wiederverheiratet & 0 & $1(3,6)$ & $1(1,2)$ \\
\cline { 2 - 5 } & getrennt lebend & $2(3,6)$ & $2(7,1)$ & $4(4,8)$ \\
\cline { 2 - 5 } & geschieden & $10(18,2)$ & $6(21,4)$ & $16(19,3)$ \\
\cline { 2 - 5 } aktuelle & ledig & $38(69,1)$ & $15(53,6)$ & $53(63,9)$ \\
\cline { 2 - 5 } Wohnsituation & mit Lebensgefährte u. Kind & $5(9,1)$ & $2(7,1)$ & $7(8,4)$ \\
\cline { 2 - 5 } & mit Kebensgefährte allein & $14(25,5)$ & $11(39,3)$ & $25(30,1)$ \\
\cline { 2 - 5 } & mit Eltern & 0 & $4(14,3)$ & $4(4,8)$ \\
\cline { 2 - 5 } & mit Familienangehörigen & $4(7,3)$ & 0 & $9(10,8)$ \\
\cline { 2 - 5 } & mit Freunden & $2(3,6)$ & $3(10,7)$ & $5(6,0)$ \\
\cline { 2 - 5 } & alleine & $19(34,5)$ & $4(14,3)$ & $23(27,7)$ \\
\cline { 2 - 5 } & keine feste Wohnsituation & $5(9,1)$ & $1(3,6)$ & $6(7,2)$ \\
\hline
\end{tabular}

Über $60 \%$ der Patienten waren ledig, wobei die Männer in dieser Gruppe stärker vertreten sind. Dieser Unterschied wurde aber nicht signifikant $(p=0,164)$. Betrachtete man die aktuelle Wohnsituation, so zeigte sich, dass Frauen in rund $40 \%$ der Fälle mit dem Lebensgefährten zusammen lebten; über ein Drittel der befragten Männer lebte alleine.

Die folgende Tabelle stellt den sozialen Status der Herkunftsfamilie, kategorisiert nach Einkommen (EK), dar.

Tabelle 4: Sozialer Status der Herkunftsfamilie

\begin{tabular}{llll}
\hline Berufsgruppe der Eltern & Männer (\%) & Frauen (\%) & Gesamt (\%) \\
\hline Arbeiter/niedriges EK & $15(27,3)$ & $5(17,9)$ & $20(24,1)$ \\
\hline Angestellte/ mittleres EK & $18(32,7)$ & $14(50,0)$ & $32(38,6)$ \\
\hline Angestellte/ hohes EK & $9(16,4)$ & $5(17,9)$ & $14(16,9)$ \\
\hline Selbständige/ mittleres EK & $6(10,9)$ & $4(14,3)$ & $10(12,0)$ \\
\hline Selbständige/ hohes EK & $5(9,1)$ & 0 & $5(6,0)$ \\
\hline k.A. & $2(3,6)$ & 0 & $2(2,4)$ \\
\hline
\end{tabular}


Rund die Hälfte der Patienten stammte demnach aus Familien mit mittlerem Einkommen, etwa jeweils ein Viertel kam aus Arbeiterfamilien mit eher geringem Einkommen und aus Familien mit hohem Einkommen.

Für die Beurteilung der Drogenkarriere der Patienten interessierte auch die Frage, in welcher Familiensituation sie in den Lebensabschnitten bis zum 6. Lebensjahr und danach überwiegend aufgewachsen waren. Hier wurde deutlich, dass der Anteil der Kinder, die vor dem 6. Lebensjahr bei ihren leiblichen Eltern aufwuchsen, nach dem 6. Lebensjahr von 68,7\% auf 53\% zurückging. Knapp $30 \%$ der befragten Patienten erlebten also im oben genannten Zeitraum eine Änderung in ihrem familienbiographischen Kontext.

Tabelle 5: Familienbiographische Situation

\begin{tabular}{|c|c|c|c|c|}
\hline & & Männer (\%) & Frauen (\%) & Gesamt (\%) \\
\hline \multirow{7}{*}{$\begin{array}{l}\text { Aufgewachsen bis } \\
\text { zum 6. Lebensjahr }\end{array}$} & Leibl. Eltern & $39(70,9)$ & $18(64,3)$ & $57(68,7)$ \\
\hline & Leibl. Mutter & $9(16,4)$ & $3(10,7)$ & $12(14,5)$ \\
\hline & Leibl. Vater & 0 & $1(3,6)$ & $1(1,2)$ \\
\hline & Sonstige Verwandte & $2(3,6)$ & 0 & $2(2,4)$ \\
\hline & Adoptiv-/Pflegeeltern & $1(1,8)$ & $4(14,3)$ & $5(6,0)$ \\
\hline & Heim & $3(5,5)$ & $1(3,6)$ & $4(4,8)$ \\
\hline & k.A. & $1(1,8)$ & $1(3,6)$ & $2(2,4)$ \\
\hline \multirow{7}{*}{$\begin{array}{l}\text { Aufgewachsen nach } \\
\text { dem 6. Lebensjahr }\end{array}$} & Leibl. Eltern & $29(52,7)$ & $15(53,6)$ & $44(53,0)$ \\
\hline & Leibl. Mutter & $17(30,9)$ & $3(10,7)$ & $19(22,9)$ \\
\hline & Leibl. Vater & $1(1,8)$ & $1(3,6)$ & $2(2,4)$ \\
\hline & Sonstige Verwandte & $2(3,6)$ & 0 & $2(2,4)$ \\
\hline & Adoptiv-/Pflegeeltern & $1(1,8)$ & $2(7,1)$ & $3(3,6)$ \\
\hline & Heim & $5(9,1)$ & $3(10,7)$ & $8(9,6)$ \\
\hline & k.A. & $2(3,6)$ & $3(10,7)$ & $5(6,0)$ \\
\hline \multirow{3}{*}{$\begin{array}{l}\text { Wechsel der } \\
\text { Bezugspersonen }\end{array}$} & ja & $18(32,7)$ & $6(21,4)$ & $24(28,9)$ \\
\hline & nein & $34(61,8)$ & $19(67,9)$ & $53(63,9)$ \\
\hline & k.A. & $3(5,5)$ & $3(10,7)$ & $6(7,2)$ \\
\hline
\end{tabular}


Hinsichtlich der Familienbiographie interessierten auch die Fragen, ob bis zum 6 . Lebensjahr eine länger dauernde Trennung von den Eltern stattgefunden hatte, ob der Patient noch weitere Geschwister hat und ob er ein Wunschkind war. Die Ergebnisse dazu sind in Tabelle 6 dargestellt.

Tabelle 6: Weitere familienbiographische Merkmale

\begin{tabular}{lllll}
\hline & & Männer (\%) & Frauen (\%) & $\begin{array}{l}\text { Gesamt } \\
(\%)\end{array}$ \\
\hline $\begin{array}{l}\text { Längere Trennung } \\
\text { von den Eltern }\end{array}$ & ja & $18(32,7)$ & $7(25,0)$ & $25(30,1)$ \\
\cline { 2 - 5 } Gein & $37(67,3)$ & $21(75,0)$ & $58(69.9)$ \\
\hline \multirow{2}{*}{ Wunschkind } & ja & $42(76,4)$ & $6(21,4)$ & $64(77,1)$ \\
\cline { 2 - 5 } & nein & $13(23,6)$ & $22(78,6)$ & $19(22,9)$ \\
\hline & ja & $30(54,5)$ & $15(53,6)$ & $45(54,2)$ \\
\hline & nein & $19(34,5)$ & $9(32,1)$ & $28(33,7)$ \\
\hline
\end{tabular}

Im Folgenden wird kurz aufgezeigt, inwieweit eine familiäre Belastung hinsichtlich der Drogenproblematik bestand. In der Erhebung wurde zwischen Verwandten väterlicher- und mütterlicherseits unterschieden; diese Unterscheidung wird hier wegen der relativ geringen Häufigkeiten nicht vorgenommen. Aus dem gleichen Grund wird in der nachfolgenden Tabelle nicht nach Geschlechtern unterschieden.

Tabelle 7: Prävalenz von familiärer Drogenabhängigkeit

\begin{tabular}{ll}
\hline Drogenabhängigkeit bekannt & Gesamt (\%) \\
\hline Großeltern & $7(8,4)$ \\
\hline Eltern & $12(14,5)$ \\
\hline Tante - Onkel & $11(13,3)$ \\
\hline wichtige andere Verwandte & $6(7,2)$ \\
\hline Brüder & $21(25,3)$ \\
\hline Schwestern & $11(13,3)$ \\
\hline Stiefgeschwister & $3(3,6)$ \\
\hline
\end{tabular}




\subsubsection{Allgemeiner Missbrauch}

In der folgenden Tabelle werden die in der Kindheit erlebten Missbrauchsformen zum einen für die Gesamtgruppe und zum anderen geschlechtsspezifisch dargestellt. Die Angaben in den Klammern hinter den Absolutzahlen sind im Folgenden immer Prozentangaben.

Tabelle 8: Häufigkeit verschiedener Missbrauchsformen

\begin{tabular}{lllll}
\hline Missbrauchsformen & $\begin{array}{l}\text { Männer } \\
(\mathbf{n = 5 5 )}\end{array}$ & $\begin{array}{l}\text { Frauen } \\
(\mathbf{n = 2 8})\end{array}$ & $\mathbf{p}$ & $\begin{array}{l}\text { Gesamt } \\
(\%)\end{array}$ \\
\hline Sexueller Missbrauch & $11(20,0)$ & $16(57,1)$ & $\mathbf{0 , 0 0 1}$ & $27(32,5)$ \\
\hline Körperliche Misshandlung & $28(50,9)$ & $17(60,7)$ & 0,397 & $45(54,2)$ \\
\hline Vernachlässigung & $34(61,8)$ & $22(78,6)$ & 0,123 & $56(67,5)$ \\
\hline Familiäre Gewalt & $26(47,3)$ & $16(57,1)$ & 0,395 & $42(50,6)$ \\
\hline Emotionale Misshandlung & $36(65,5)$ & $19(67,9)$ & 0,583 & $55(66,3)$ \\
\hline Missbrauch jeglicher Art & $42(76,4)$ & $26(92,9)$ & 0,065 & $68(81,9)$ \\
\hline Mann-Whitney-U-Test, 2-seitig & & & &
\end{tabular}

Bei der Frage nach „Missbrauch jeglicher Art", die dann bejaht wurde, wenn auch nur eine der fünf Kategorien angegeben wurde, zeigte sich, dass über $80 \%$ der Studienpopulation über entsprechende Erfahrungen verfügten. Geschlechtsspezifische Unterschiede wurden nur beim sexuellen Missbrauch signifikant. Dennoch war auffällig, dass die Frauen bei allen Missbrauchsarten stärker betroffen waren als die Männer.

Es stellte sich die Frage, wie sich die Gruppen mit und ohne Missbrauchserfahrung hinsichtlich der neun Untergruppen des EuropASI unterscheiden. Betrachtet wurde dazu für jede Untergruppe der Mittelwert des errechneten Composite Score, der mittlere Rang sowie der p-Wert, der mittels des Mann-Whitney-Tests die Signifikanz bezüglich eines Unterschieds der Ergebniswerte der beiden Gruppen misst. 
Ein signifikanter Unterschied zwischen den beiden Gruppen fand sich demnach in den Bereichen körperliche und psychische Probleme mit einer stärkeren Belastung der Personen mit Missbrauchserfahrungen. Im Folgenden soll nicht jedes Mal erneut darauf hingewiesen werden, dass die stärkere Belastung bei der missbrauchten Personengruppe liegt. Sollte sich dieser Zusammenhang einmal umkehren, wird selbstverständlich darauf hingewiesen.

Tabelle 9: Lagemaße der Composite Scores des EuropASI nach allgemeiner Missbrauchserfahrung

\begin{tabular}{|c|c|c|c|c|}
\hline $\begin{array}{l}\text { Composite Scores nach } \\
\text { EuropASI }\end{array}$ & & $\begin{array}{l}\text { Personen mit } \\
\text { Missbrauchserf. } \\
(n=68)\end{array}$ & $\begin{array}{l}\text { Personen ohne } \\
\text { Missbrauchserf } \\
(n=15)\end{array}$ & p-Wert \\
\hline \multirow[b]{2}{*}{ Körperliche Probleme } & $\mathrm{MW}$ & 0,23 & 0,02 & \multirow[b]{2}{*}{0,013} \\
\hline & $\begin{array}{l}\text { Mitt. } \\
\text { Rang }\end{array}$ & 44,59 & 30,27 & \\
\hline \multirow[b]{2}{*}{ Arbeit: Ökon. Situation } & MW & 0,79 & 0,74 & \multirow[b]{2}{*}{0,582} \\
\hline & $\begin{array}{l}\text { Mitt. } \\
\text { Rang }\end{array}$ & 42,53 & 39,60 & \\
\hline \multirow[b]{2}{*}{ Arbeit: Zufriedenheit } & MW & 0,27 & 0,33 & \multirow[b]{2}{*}{0,832} \\
\hline & $\begin{array}{l}\text { Mitt. } \\
\text { Rang }\end{array}$ & 41,76 & 43,10 & \\
\hline \multirow[b]{2}{*}{ Alkoholgebrauch } & MW & 0,18 & 0,24 & \multirow[b]{2}{*}{0,800} \\
\hline & $\begin{array}{l}\text { Mitt. } \\
\text { Rang }\end{array}$ & 41,74 & 43,20 & \\
\hline \multirow[b]{2}{*}{ Drogengebrauch } & MW & 0,68 & 0,69 & \multirow[b]{2}{*}{0,868} \\
\hline & $\begin{array}{l}\text { Mitt. } \\
\text { Rang }\end{array}$ & 42,21 & 41,07 & \\
\hline \multirow[b]{2}{*}{ Rechtliche Probleme } & $\mathrm{MW}$ & 0,35 & 0,35 & \multirow[b]{2}{*}{0,981} \\
\hline & $\begin{array}{l}\text { Mitt. } \\
\text { Rang }\end{array}$ & 42,03 & 41,87 & \\
\hline \multirow[b]{2}{*}{ Familie } & MW & 0,29 & 0,23 & \multirow[b]{2}{*}{0,658} \\
\hline & $\begin{array}{l}\text { Mitt. } \\
\text { Rang }\end{array}$ & 42,53 & 39,60 & \\
\hline
\end{tabular}




\begin{tabular}{lllll}
\hline \multirow{2}{*}{ Sozialbeziehungen } & \multicolumn{1}{l}{ MW } & 0,18 & 0,04 & \multirow{2}{*}{0,075} \\
\cline { 2 - 4 } & $\begin{array}{l}\text { Mitt. } \\
\text { Rang }\end{array}$ & 43,99 & 33,00 & \\
\hline \multirow{2}{*}{ Psychische Probleme } & MW & 0,34 & 0,006 \\
\cline { 2 - 4 } & Mitt. & 45,40 & 26,60 & \\
\hline & Rang & & & \\
\hline
\end{tabular}

Mann-Whitney-U-Test, 2-seitig

Es wurde ebenfalls überprüft, inwieweit sich aus dem SCL 90-R Unterschiede in den Skalenwerten (Tabelle 10) und den drei globalen Kennwerten GSI, PST und PSDI (Tabelle 11) ergeben. Hier wurden die Skalenwerte Somatisierung, Depressivität, Ängstlichkeit, Aggressivität, phobische Angst, paranoides Denken und Psychotizismus signifikant. Ebenso signifikant waren die Ergebnisse für die drei globalen Kennwerte. Es zeigte sich hier, dass sich die Gruppen mit und ohne eine Missbrauchserfahrung in einer Vielzahl von Symptombelastungen unterschieden.

Tabelle 10: Lagemaße der Skalenwerte des SCL 90-R nach allgemeiner Missbrauchserfahrung

\begin{tabular}{|c|c|c|c|c|}
\hline $\begin{array}{l}\text { Skalenwerte nach } \\
\text { SCL } 90-R\end{array}$ & & $\begin{array}{l}\text { Personen mit } \\
\text { Missbrauchserf. } \\
(n=68)\end{array}$ & $\begin{array}{l}\text { Personen ohne } \\
\text { Missbrauchserf } \\
(n=15)\end{array}$ & p-Wert \\
\hline \multirow{2}{*}{ Somatisierung } & $\mathrm{MW}$ & 1,05 & 0,59 & \multirow{2}{*}{0,010} \\
\hline & Mitt. Rang & 45,19 & 27,53 & \\
\hline \multirow{2}{*}{ Zwanghaftigkeit } & MW & 1,21 & 0,88 & \multirow{2}{*}{0,158} \\
\hline & Mitt. Rang & 43,75 & 34,07 & \\
\hline \multirow{2}{*}{$\begin{array}{l}\text { Unsicherheit } \\
\text { Sozialkontakt }\end{array}$} & MW & 0,95 & 0,55 & \multirow{2}{*}{0,084} \\
\hline & Mitt. Rang & 44,14 & 32,30 & \\
\hline \multirow{2}{*}{ Depressivität } & MW & 1,36 & 0,82 & \multirow{2}{*}{0,021} \\
\hline & Mitt. Rang & 44,87 & 29,00 & \\
\hline \multirow{2}{*}{ Ängstlichkeit } & MW & 1,19 & 0,55 & \multirow{2}{*}{0,010} \\
\hline & Mitt. Rang & 45,18 & 27,57 & \\
\hline \multirow{2}{*}{ Aggressivität } & $\mathrm{MW}$ & 1,09 & 0,80 & \multirow{2}{*}{0,048} \\
\hline & Mitt. Rang & 44,44 & 30,93 & \\
\hline
\end{tabular}




\begin{tabular}{lllll}
\hline \multirow{2}{*}{ Phobische Angst } & MW & 0,53 & 0,16 & \multirow{2}{*}{$\mathbf{0 , 0 4 0}$} \\
\cline { 2 - 4 } & Mitt. Rang & 44,48 & 30,77 & \multirow{2}{*}{$\mathbf{0 , 0 4 0}$} \\
\cline { 1 - 4 } Paranoides Denken & MW & 1,03 & 0,56 & \multirow{2}{*}{$\mathbf{0 , 0 1 1}$} \\
\cline { 2 - 4 } & Mitt. Rang & 44,54 & 30,47 & 0,19 \\
\hline \multirow{2}{*}{ Psychotizismus } & MW & 0,62 & 27,87 & \\
\cline { 2 - 3 } & Mitt. Rang & 45,12 & \\
\hline
\end{tabular}

Mann-Whitney-U-Test, 2-seitig

Tabelle 11: Lagemaße der Globalen Kennwerte des SCL 90-R nach allgemeiner Missbrauchserfahrung

\begin{tabular}{lllll}
\hline $\begin{array}{l}\text { Globale Kennwerte nach } \\
\text { SCL 90-R }\end{array}$ & & $\begin{array}{l}\text { Personen mit } \\
\text { Missbrauchserf. } \\
(\mathbf{n}=68)\end{array}$ & $\begin{array}{l}\text { Personen ohne } \\
\text { Missbrauchserf } \\
(\mathbf{n}=\mathbf{1 5})\end{array}$ & p-Wert \\
\hline \multirow{2}{*}{ GSI } & MW & 1,04 & 0,59 & \multirow{2}{*}{$\mathbf{0 , 0 1 2}$} \\
\cline { 2 - 4 } & Mitt. Rang & 45,12 & 27,87 & \multirow{2}{*}{$\mathbf{0 , 0 2 1}$} \\
\hline \multirow{2}{*}{ PSDI } & MW & 2,01 & 1,63 & \multirow{2}{*}{$\mathbf{0 , 0 1 4}$} \\
\cline { 2 - 5 } & Mitt. Rang & 44,86 & 29,03 & \\
\hline \multirow{2}{*}{ PST } & MW & 43,13 & 28,86 & 28,17 \\
\hline
\end{tabular}

Mann-Whitney-U-Test, 2-seitig

\subsubsection{Sexueller Missbrauch}

Wie bereits in Tabelle 8 aufgeführt, gaben 32,5\% aller Studienteilnehmer an, sexuell missbraucht worden zu sein, wobei die Frauen mit einem Anteil von rund $57 \%(p=0,001)$ diese Frage signifikant häufiger bejahten.

Die Auswertung der Composite Scores in Tabelle 12 wies lediglich in den Punkten körperliche Probleme und psychische Probleme einen signifikanten Unterschied auf. 
Tabelle 12: Lagemaße der Composite Scores des EuropASI nach sexueller Missbrauchserfahrung

\begin{tabular}{|c|c|c|c|c|}
\hline $\begin{array}{l}\text { Composite Scores nach } \\
\text { EuropASI }\end{array}$ & & $\begin{array}{l}\text { Personen mit } \\
\text { Missbrauchserf. } \\
(\mathrm{n}=27)\end{array}$ & $\begin{array}{l}\text { Personen ohne } \\
\text { Missbrauchserf } \\
(n=56)\end{array}$ & p-Wert \\
\hline & MW & 0,32 & 0,14 & \\
\hline Körperliche Probleme & $\begin{array}{l}\text { Mitt. } \\
\text { Rang }\end{array}$ & 48,39 & 38,92 & 0,046 \\
\hline & $\mathrm{MW}$ & 0,74 & 0,81 & \\
\hline Arbeit: Ökon. Situation & $\begin{array}{l}\text { Mitt. } \\
\text { Rang }\end{array}$ & 40,20 & 42,87 & 0,543 \\
\hline & MW & 0,31 & 0,26 & \\
\hline Arbeit: Zufriedenheit & $\begin{array}{l}\text { Mitt. } \\
\text { Rang }\end{array}$ & 43,96 & 41,05 & 0,576 \\
\hline & MW & 0,14 & 0,21 & \\
\hline Alkoholgebrauch & $\begin{array}{l}\text { Mitt. } \\
\text { Rang }\end{array}$ & 40,44 & 42,75 & 0,628 \\
\hline & MW & 0,68 & 0,69 & \\
\hline Drogengebrauch & $\begin{array}{l}\text { Mitt. } \\
\text { Rang }\end{array}$ & 44,54 & 40,78 & 0,506 \\
\hline & MW & 0,35 & 0,35 & \\
\hline Rechtliche Probleme & $\begin{array}{l}\text { Mitt. } \\
\text { Rang }\end{array}$ & 40,37 & 42,79 & 0,665 \\
\hline Familie & $\begin{array}{l}\text { MW } \\
\text { Mitt. } \\
\text { Rang }\end{array}$ & $\begin{array}{l}0,29 \\
42,04\end{array}$ & $\begin{array}{l}0,27 \\
41,98\end{array}$ & 0,992 \\
\hline Sozialbeziehungen & $\begin{array}{l}\text { MW } \\
\text { Mitt. } \\
\text { Rang }\end{array}$ & $\begin{array}{l}0,20 \\
45,61\end{array}$ & $\begin{array}{l}0,13 \\
40,26\end{array}$ & 0,291 \\
\hline Psychische Probleme & $\begin{array}{l}\text { MW } \\
\text { Mitt. } \\
\text { Rang }\end{array}$ & $\begin{array}{l}0,43 \\
54,74\end{array}$ & $\begin{array}{l}0,25 \\
35,86\end{array}$ & 0,001 \\
\hline
\end{tabular}

Mann-Whitney-U-Test, 2-seitig

Bei den Ergebnissen des SCL 90-R war sehr auffällig, dass alle Skalenwerte mit Ausnahme der Zwanghaftigkeit und Aggressivität signifikant wurden. 
Hochsignifikant erwiesen sich die Werte für die Skalen Unsicherheit im Sozialkontakt und Depressivität. Bei den globalen Kennwerte des SCL 90-R waren der GSI signifikant, der PST hochsignifikant. Anhand der Ergebnisse wird deutlich, dass sich die Gruppen mit und ohne eine sexuelle Missbrauchserfahrung in der psychischen Symptombelastung stark unterscheiden.

Tabelle 13: Lagemaße der Skalenwerte des SCL 90-R nach sexueller Missbrauchserfahrung

\begin{tabular}{|c|c|c|c|c|}
\hline $\begin{array}{l}\text { Skalenwerte nach } \\
\text { SCL 90-R }\end{array}$ & & $\begin{array}{l}\text { Personen mit } \\
\text { Missbrauchserf. } \\
(\mathrm{n}=27)\end{array}$ & $\begin{array}{l}\text { Personen ohne } \\
\text { Missbrauchserf } \\
(n=56)\end{array}$ & p-Wert \\
\hline \multirow{2}{*}{ Somatisierung } & MW & 1,20 & 0,86 & \multirow{2}{*}{0,060} \\
\hline & Mitt. Rang & 49,15 & 38,55 & \\
\hline \multirow{2}{*}{ Zwanghaftigkeit } & $\mathrm{MW}$ & 1,44 & 1,01 & \multirow{2}{*}{0,054} \\
\hline & Mitt. Rang & 49,31 & 38,47 & \\
\hline \multirow{2}{*}{$\begin{array}{l}\text { Unsicherheit } \\
\text { Sozialkontakt }\end{array}$} & MW & 1,29 & 0,67 & \multirow{2}{*}{0,001} \\
\hline & Mitt. Rang & 54,89 & 35,79 & \\
\hline \multirow{2}{*}{ Depressivität } & $\mathrm{MW}$ & 1,74 & 1,03 & \multirow{2}{*}{0,001} \\
\hline & Mitt. Rang & 54,56 & 35,95 & \\
\hline \multirow{2}{*}{ Ängstlichkeit } & $\mathrm{MW}$ & 1,36 & 0,9380 & \multirow{2}{*}{0,004} \\
\hline & Mitt. Rang & 52,91 & 36,74 & \\
\hline \multirow{2}{*}{ Aggressivität } & $\mathrm{MW}$ & 1,20 & 0,95 & \multirow{2}{*}{0,069} \\
\hline & Mitt. Rang & 48,89 & 38,68 & \\
\hline \multirow{2}{*}{ Phobische Angst } & MW & 0,72 & 0,34 & \multirow{2}{*}{0,013} \\
\hline & Mitt. Rang & 51,17 & 37,58 & \\
\hline \multirow{2}{*}{ Paranoides Denken } & $\mathrm{MW}$ & 1,31 & 0,77 & \multirow{2}{*}{0,011} \\
\hline & Mitt. Rang & 51,59 & 37,38 & \\
\hline \multirow{2}{*}{ Psychotizismus } & MW & 0,89 & 0,37 & \multirow{2}{*}{0,007} \\
\hline & Mitt. Rang & 52,11 & 37,13 & \\
\hline
\end{tabular}

Mann-Whitney-U-Test, 2-seitig

Betrachtete man die Mittelwerte der Skalenwerte und der drei globalen Kennwerte in der Gruppe mit sexueller Missbrauchserfahrung, so war 
festzustellen, dass die absolute Belastung dieser Patienten am höchsten im Vergleich mit allen anderen Missbrauchsgruppen war (siehe auch Tabelle 61 und Tabelle 62). Dies deutete darauf hin, dass diese Missbrauchsgruppe von den Folgen der Traumatisierung am stärksten betroffen war und sich dies in der gemessenen durchschnittlichen psychischen Symptombelastung niederschlug.

Tabelle 14: Lagemaße der Globalen Kennwerte des SCL 90-R nach sexueller Missbrauchserfahrung

\begin{tabular}{|c|c|c|c|c|}
\hline $\begin{array}{l}\text { Globale Kennwerte nach } \\
\text { SCL } 90-R\end{array}$ & & $\begin{array}{l}\text { Personen mit } \\
\text { Missbrauchserf. } \\
(n=27)\end{array}$ & $\begin{array}{l}\text { Personen ohne } \\
\text { Missbrauchserf } \\
(n=56)\end{array}$ & p-Wert \\
\hline \multirow{2}{*}{ GSI } & MW & 1,29 & 0,80 & \multirow{2}{*}{0,004} \\
\hline & Mitt. Rang & 53,00 & 36,70 & \\
\hline \multirow{2}{*}{ PSDI } & $\mathrm{MW}$ & 2,10 & 1,87 & \multirow{2}{*}{0,069} \\
\hline & Mitt. Rang & 48,93 & 38,66 & \\
\hline \multirow{2}{*}{ PST } & MW & 51,07 & 35,48 & \multirow{2}{*}{0,001} \\
\hline & Mitt. Rang & 54,26 & 36,09 & \\
\hline
\end{tabular}

Mann-Whitney-U-Test, 2-seitig

Als weiteres stellte sich die Frage, inwieweit sich bei den Patienten mit sexueller Missbrauchserfahrung geschlechtsspezifische Unterschiede finden lassen. Dazu wurden die Composite Scores nach EuropASI, die Skalenwerte und die globalen Kennwerte nach SCL 90-R mittels des Chi-Quadrat Tests nach Pearson analysiert. In den folgenden Tabellen sind der Chi-Wert, der Freiheitsgrad sowie der p-Wert für die Signifikanz angegeben.

Wie aus Tabelle 15, Tabelle 16 und Tabelle 17 ersichtlich ist, fanden sich keine geschlechtsspezifischen signifikanten Unterschiede bezüglich der Kennwerte. D.h. Männer und Frauen, die eine sexuelle Missbrauchserfahrung in der Kindheit erlitten haben, waren demnach in ihrer Belastung bezüglich der Problembereiche und der psychischen Symptome äquivalent betroffen. 
Tabelle 15: Geschlechtsspezifische Unterschiede für die Composite Scores des EuropASI nach sexueller Missbrauchserfahrung

\begin{tabular}{llll}
\hline $\begin{array}{l}\text { Composite Scores nach } \\
\text { EuropASI }\end{array}$ & $\chi$-Wert & $\begin{array}{l}\text { Freiheits- } \\
\text { grad }\end{array}$ & p-Wert \\
\hline Körperliche Probleme & 11,536 & 10 & 0,317 \\
& & & \\
\hline Arbeit: Ökon. Situation & 2,293 & 3 & 0,514 \\
\hline Arbeit: Zufriedenheit & 10,697 & 11 & 0,469 \\
\hline Alkoholgebrauch & 10,432 & 9 & 0,317 \\
\hline Drogengebrauch & 27,000 & 26 & 0,409 \\
\hline Rechtliche Probleme & 8,863 & 9 & 0,450 \\
\hline Familie & 12,848 & 14 & 0,539 \\
\hline Sozialbeziehungen & 9,544 & 10 & 0,481 \\
\hline Psychische Probleme & 21,477 & 22 & 0,491 \\
\hline
\end{tabular}

Chi-Quadrat-Test, 2-seitig, nach Pearson

Tabelle 16: Geschlechtsspezifische Unterschiede für die Skalenwerten des SCL 90-R nach sexueller Missbrauchserfahrung

\begin{tabular}{llll}
\hline $\begin{array}{l}\text { Skalenwerte nach } \\
\text { SCL 90-R }\end{array}$ & $\chi$-Wert & $\begin{array}{l}\text { Freiheits- } \\
\text { grad }\end{array}$ & p-Wert \\
\hline Somatisierung & 14,574 & 16 & 0,556 \\
\hline Zwanghaftigkeit & 14,919 & 15 & 0,457 \\
\hline Unsicherheit Sozialkontakt & 20,097 & 17 & 0,269 \\
\hline Depressivität & 20,097 & 21 & 0,515 \\
\hline Ängstlichkeit & 17,335 & 17 & 0,432 \\
\hline Aggressivität & 16,645 & 13 & 0,216 \\
\hline Phobische Angst & 17,128 & 12 & 0,145 \\
\hline Paranoides Denken & 12,848 & 15 & 0,614 \\
\hline Psychotizismus & 17,887 & 15 & 0,269 \\
\hline
\end{tabular}

Chi-Quadrat-Test, 2-seitig, nach Pearson 
Tabelle 17: Geschlechtsspezifische Unterschiede für die Globalen Kennwerte des SCL 90R nach sexueller Missbrauchserfahrung

\begin{tabular}{llll}
\hline $\begin{array}{l}\text { Globale Kennwerte nach } \\
\text { SCL 90-R }\end{array}$ & $\chi$-Wert & $\begin{array}{l}\text { Freiheits- } \\
\text { grad }\end{array}$ & p-Wert \\
\hline GSI & 24,929 & 24 & 0,410 \\
\hline PSDI & 27,000 & 26 & 0,409 \\
\hline PST & 20,787 & 22 & 0,534 \\
\hline
\end{tabular}

Chi-Quadrat-Test, 2-seitig, nach Pearson

\subsubsection{Körperliche Misshandlung}

Insgesamt gaben rund 54\% der Befragten eine körperliche Misshandlung an. Auch hier waren die Frauen wieder stärker betroffen; der Unterschied wurde allerdings nicht signifikant. Die Daten wurden wieder in zwei Gruppen mit und ohne körperlichen Missbrauch aufgeteilt und auf Unterschiede untersucht.

Das Ergebnis der Problembereiche nach EuropASI zeigt Tabelle 18. Für die Skalen körperliche Probleme, Sozialbeziehungen und psychische Probleme zeigte die Gruppe der Patienten mit körperlicher Missbrauchserfahrung signifikant höhere Werte.

Tabelle 18: Lagemaße der Composite Scores des EuropASI nach körperlicher Missbrauchserfahrung

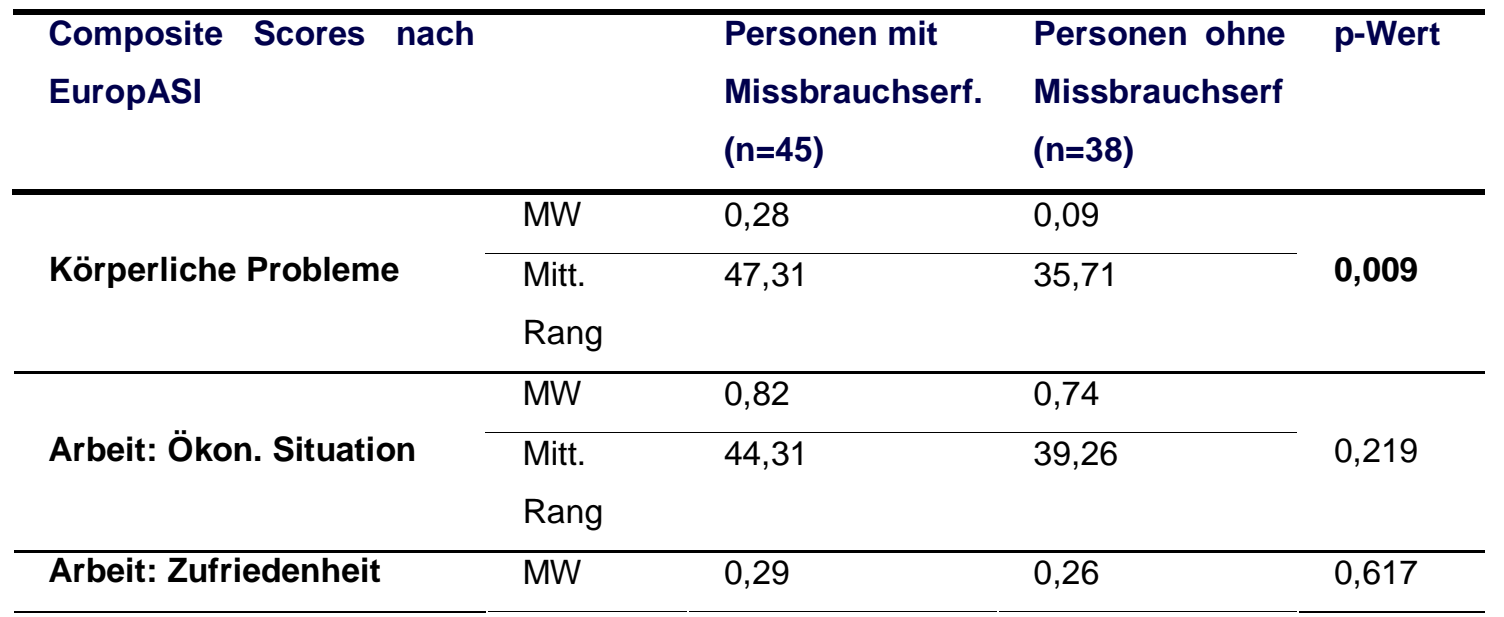




\begin{tabular}{|c|c|c|c|c|}
\hline & Mitt. & 43,12 & 40,67 & \\
\hline & Rang & & & \\
\hline \multirow{3}{*}{ Alkoholgebrauch } & MW & 0,21 & 0,17 & \multirow{3}{*}{0,334} \\
\hline & Mitt. & 43,98 & 39,66 & \\
\hline & Rang & & & \\
\hline \multirow{3}{*}{ Drogengebrauch } & MW & 0,71 & 0,66 & \multirow{3}{*}{0,385} \\
\hline & Mitt. & 44,11 & 39,50 & \\
\hline & Rang & & & \\
\hline \multirow{3}{*}{ Rechtliche Probleme } & MW & 0,38 & 0,31 & \multirow{3}{*}{0,292} \\
\hline & Mitt. & 44,53 & 39,00 & \\
\hline & Rang & & & \\
\hline \multirow{3}{*}{ Familie } & MW & 0,34 & 0,21 & \multirow{3}{*}{0,072} \\
\hline & Mitt. & 46,20 & 37,03 & \\
\hline & Rang & & & \\
\hline \multirow{3}{*}{ Sozialbeziehungen } & MW & 0,23 & 0,07 & \multirow{3}{*}{0,006} \\
\hline & Mitt. & 48,03 & 34,86 & \\
\hline & Rang & & & \\
\hline \multirow{3}{*}{ Psychische Probleme } & MW & 0,37 & 0,24 & \multirow{3}{*}{0,007} \\
\hline & Mitt. & 48,58 & 34,21 & \\
\hline & Rang & & & \\
\hline
\end{tabular}

Mann-Whitney-U-Test, 2-seitig

Beim SCL 90-R wurden deutlich weniger Skalenwerte signifikant als bei der Aufteilung nach sexuellem Missbrauch. Die Skalenwerte zeigten für die beiden Gruppen bis auf paranoides Denken keine signifikanten Unterschiede. Auch die globalen Kennwerte waren nicht auffällig.

Tabelle 19: Lagemaße der Skalenwerte des SCL 90-R nach körperlicher Missbrauchserfahrung

\begin{tabular}{lllll}
\hline $\begin{array}{l}\text { Skalenwerte nach } \\
\text { SCL 90-R }\end{array}$ & $\begin{array}{l}\text { Personen mit } \\
\text { Missbrauchserf. } \\
(\mathbf{n}=\mathbf{4 5})\end{array}$ & $\begin{array}{l}\text { Personen ohne } \\
\text { Missbrauchserf } \\
(\mathbf{n}=\mathbf{3 8})\end{array}$ & p-Wert \\
\hline Somatisierung & MW & 1,09 & 0,83 & 0,110 \\
\cline { 2 - 5 } & Mitt. Rang & 45,88 & 37,41 & 0,570 \\
\hline Zwanghaftigkeit & MW & 1,22 & 1,07 & \\
\hline
\end{tabular}




\begin{tabular}{|c|c|c|c|c|}
\hline & Mitt. Rang & 43,38 & 40,37 & \\
\hline \multirow{2}{*}{$\begin{array}{l}\text { Unsicherheit } \\
\text { Sozialkontakt }\end{array}$} & MW & 0,99 & 0,74 & \multirow{2}{*}{0,273} \\
\hline & Mitt. Rang & 44,66 & 38,86 & \\
\hline \multirow{2}{*}{ Depressivität } & MW & 1,39 & 1,10 & \multirow{2}{*}{0,135} \\
\hline & Mitt. Rang & 45,63 & 37,70 & \\
\hline \multirow{2}{*}{ Ängstlichkeit } & MW & 1,26 & 0,84 & \multirow{2}{*}{0,125} \\
\hline & Mitt. Rang & 45,72 & 37,59 & \\
\hline \multirow{2}{*}{ Aggressivität } & MW & 1,11 & 0,94 & \multirow{2}{*}{0,153} \\
\hline & Mitt. Rang & 45,46 & 37,91 & \\
\hline \multirow{2}{*}{ Phobische Angst } & MW & 0,55 & 0,36 & \multirow{2}{*}{0,330} \\
\hline & Mitt. Rang & 44,30 & 39,28 & \\
\hline \multirow{2}{*}{ Paranoides Denken } & MW & 1,14 & 0,72 & \multirow{2}{*}{0,032} \\
\hline & Mitt. Rang & 47,18 & 35,87 & \\
\hline \multirow{2}{*}{ Psychotizismus } & MW & 0,65 & 0,41 & \multirow{2}{*}{0,156} \\
\hline & Mitt. Rang & 45,41 & 37,96 & \\
\hline
\end{tabular}

Mann-Whitney-U-Test, 2-seitig

Tabelle 20: Lagemaße der Globalen Kennwerte des SCL 90-R nach körperlicher Missbrauchserfahrung

\begin{tabular}{|c|c|c|c|c|}
\hline $\begin{array}{l}\text { Globale } \\
\text { nach } \\
\text { SCL 90-R }\end{array}$ & Kennwerte & $\begin{array}{l}\text { Personen mit } \\
\text { Missbrauchserf. } \\
(\mathrm{n}=45)\end{array}$ & $\begin{array}{l}\text { Personen ohne } \\
\text { Missbrauchserf } \\
(n=38)\end{array}$ & p-Wert \\
\hline \multirow{2}{*}{ GSI } & MW & 1,08 & 0,82 & \multirow{2}{*}{0,116} \\
\hline & Mitt. Rang & 45,82 & 37,47 & \\
\hline \multirow{2}{*}{ PSDI } & MW & 2,04 & 1,83 & \multirow{2}{*}{0,182} \\
\hline & Mitt. Rang & 45,24 & 38,16 & \\
\hline \multirow{2}{*}{ PST } & MW & 44,02 & 36,44 & \multirow{2}{*}{0,101} \\
\hline & Mitt. Rang & 45,99 & 37,28 & \\
\hline
\end{tabular}

Mann-Whitney-U-Test, 2-seitig

Teilte man die Gruppe der körperlich misshandelten Personen nach Geschlecht auf, so zeigten die Frauen lediglich in der Skala zu Unsicherheit im Sozialkontakt ( $p=0,031$ ) einen signifikant höheren Wert als die männlichen körperlich misshandelten Personen. Die anderen Kennwerte blieben unauffällig bezüglich einer geschlechtsspezifischen Unterscheidung. 
Tabelle 21: Geschlechtsspezifische Unterschiede für die Composite Scores des EuropASI nach körperlicher Missbrauchserfahrung

\begin{tabular}{llll}
\hline $\begin{array}{l}\text { Composite Scores nach } \\
\text { EuropASI }\end{array}$ & $\chi$-Wert & $\begin{array}{l}\text { Freiheits- } \\
\text { grad }\end{array}$ & p-Wert \\
\hline Körperliche Probleme & 20,723 & 14 & 0,109 \\
\hline Arbeit: Ökon. Situation & 2,526 & 3 & 0,471 \\
\hline Arbeit: Zufriedenheit & 12,122 & 16 & 0,736 \\
\hline Alkoholgebrauch & 17,269 & 17 & 0,436 \\
\hline Drogengebrauch & 42,873 & 43 & 0,477 \\
\hline Rechtliche Probleme & 24,535 & 21 & 0,268 \\
\hline Familie & 20,609 & 25 & 0,714 \\
\hline Sozialbeziehungen & 15,271 & 15 & 0,432 \\
\hline Psychische Probleme & 31,528 & 31 & 0,440 \\
\hline
\end{tabular}

Chi-Quadrat-Test, 2-seitig, nach Pearson

Tabelle 22: Geschlechtsspezifische Unterschiede für die Skalenwerte des SCL 90-R nach körperlicher Missbrauchserfahrung

\begin{tabular}{llll}
\hline $\begin{array}{l}\text { Skalenwerte nach } \\
\text { SCL 90-R }\end{array}$ & $\chi$-Wert & $\begin{array}{l}\text { Freiheits- } \\
\text { grad }\end{array}$ & p-Wert \\
\hline Somatisierung & 29,756 & 24 & 0,193 \\
\hline Zwanghaftigkeit & 28,125 & 24 & 0,255 \\
\hline Unsicherheit Sozialkontakt & 33,301 & 20 & $\mathbf{0 , 0 3 1}$ \\
\hline Depressivität & 27,983 & 28 & 0,465 \\
\hline Ängstlichkeit & 23,871 & 21 & 0,299 \\
\hline Aggressivität & 12,476 & 14 & 0,568 \\
\hline Phobische Angst & 21,572 & 13 & 0,062 \\
\hline Paranoides Denken & 17,525 & 17 & 0,419 \\
\hline Psychotizismus & 24,296 & 15 & 0,060 \\
\hline
\end{tabular}

Chi-Quadrat-Test, 2-seitig, nach Pearson 
Tabelle 23: Geschlechtsspezifische Unterschiede für die Globalen Kennwerte des SCL 90R nach körperlicher Missbrauchserfahrung

\begin{tabular}{llll}
\hline $\begin{array}{l}\text { Globale Kennwerte nach } \\
\text { SCL 90-R }\end{array}$ & $\chi$-Wert & $\begin{array}{l}\text { Freiheits- } \\
\text { grad }\end{array}$ & p-Wert \\
\hline GSI & 40,746 & 37 & 0,309 \\
\hline PSDI & 45,000 & 42 & 0,347 \\
\hline PST & 34,364 & 35 & 0,499 \\
\hline
\end{tabular}

Chi-Quadrat-Test, 2-seitig, nach Pearson

\subsubsection{Vernachlässigung}

Etwa $67 \%$ der Befragten gaben Vernachlässigung in der Kindheit an, wobei auch hier die Frauen wieder deutlich stärker betroffen waren, ohne dass es zu einem signifikanten Unterschied gekommen war.

Die Daten wurden wieder wie bereits in den vorangegangenen Punkten in zwei Gruppen mit und ohne Vernachlässigung in der Befragung aufgeteilt und hinsichtlich der Höhe der Composite Scores miteinander verglichen. Die resultierenden $\mathrm{p}$-Werte sind in der folgenden Tabelle aufgeführt. Die Patienten, die in der Kindheit unter Vernachlässigung litten, zeigten eine höhere Belastung bei den Symptomen bezüglich Körperlicher Probleme, ihrer Ökonomischen Situation sowie in ihren Sozialbeziehungen. Bei psychischen Problemen unterschied sich diese Gruppe sogar hochsignifikant von der Vergleichsgruppe.

Tabelle 24: Lagemaße der Composite Scores des EuropASI nach Vernachlässigung

\begin{tabular}{lllll}
\hline $\begin{array}{l}\text { Composite Scores nach } \\
\text { EuropASI }\end{array}$ & & $\begin{array}{l}\text { Personen mit } \\
\text { Missbrauchserf. } \\
(\mathbf{n}=56)\end{array}$ & $\begin{array}{l}\text { Personen ohne } \\
\text { Missbrauchserf } \\
(\mathbf{n}=\mathbf{2 7})\end{array}$ & p-Wert \\
\hline \multirow{2}{*}{ Körperliche Probleme } & MW & 0,25 & 0,09 & \multirow{0}{*}{$\mathbf{0 1 7}$} \\
\cline { 2 - 5 } & Mitt. & 45,71 & 34,31 & \\
\hline Arbeit: Ökon. Situation & MW & 0,85 & 0,013 \\
\hline
\end{tabular}




\begin{tabular}{|c|c|c|c|c|}
\hline & $\begin{array}{l}\text { Mitt. } \\
\text { Rang }\end{array}$ & 45,53 & 34,69 & \\
\hline \multirow{3}{*}{ Arbeit: Zufriedenheit } & MW & 0,31 & 0,22 & \multirow{3}{*}{0,123} \\
\hline & Mitt. & 44,62 & 36,57 & \\
\hline & Rang & & & \\
\hline \multirow{3}{*}{ Alkoholgebrauch } & $\mathrm{MW}$ & 0,19 & 0,20 & \multirow{3}{*}{0,782} \\
\hline & Mitt. & 42,43 & 41,11 & \\
\hline & Rang & & & \\
\hline \multirow{3}{*}{ Drogengebrauch } & $\mathrm{MW}$ & 0,71 & 0,63 & \multirow{3}{*}{0,120} \\
\hline & Mitt. & 44,86 & 36,07 & \\
\hline & Rang & & & \\
\hline \multirow{3}{*}{ Rechtliche Probleme } & MW & 0,37 & 0,30 & \multirow{3}{*}{0,260} \\
\hline & Mitt. & 44,04 & 37,76 & \\
\hline & Rang & & & \\
\hline \multirow{3}{*}{ Familie } & $\mathrm{MW}$ & 0,32 & 0,18 & \multirow{3}{*}{0,099} \\
\hline & Mitt. & 44,91 & 35,96 & \\
\hline & Rang & & & \\
\hline \multirow{3}{*}{ Sozialbeziehungen } & $\mathrm{MW}$ & 0,20 & 0,07 & \multirow{3}{*}{0,009} \\
\hline & Mitt. & 46,30 & 33,07 & \\
\hline & Rang & & & \\
\hline \multirow{3}{*}{ Psychische Probleme } & MW & 0,37 & 0,18 & \multirow{3}{*}{0,000} \\
\hline & Mitt. & 48,47 & 28,57 & \\
\hline & Rang & & & \\
\hline
\end{tabular}

Mann-Whitney-U-Test, 2-seitig

Bei der Analyse der Ergebnisse des SCL 90-R wurden zwischen den Gruppen mit und ohne Vernachlässigung in der Kindheit fast alle Werte signifikant. Lediglich die Skala Unsicherheit im Sozialkontakt blieb ohne einen nachweisbaren Unterschied. Einige der Skalenwerte waren sogar hochsignifikant (Somatisierung, Ängstlichkeit, Psychotizismus und PST). Die absoluten Werte der Symptombelastung, hier der Skalenwerte und der drei globalen Kennwerte, lagen allerdings im Mittel unter denen der sexuell missbrauchten Gruppe (siehe Tabelle 61 und Tabelle 62). 
Tabelle 25: Lagemaße der Skalenwerte des SCL 90-R nach Vernachlässigung

\begin{tabular}{|c|c|c|c|c|}
\hline $\begin{array}{l}\text { Skalenwerte nach } \\
\text { SCL 90-R }\end{array}$ & & $\begin{array}{l}\text { Personen mit } \\
\text { Missbrauchserf. } \\
(n=56)\end{array}$ & $\begin{array}{l}\text { Personen ohne } \\
\text { Missbrauchserf } \\
(n=27)\end{array}$ & p-Wert \\
\hline \multirow{2}{*}{ Somatisierung } & $\mathrm{MW}$ & 1,14 & 0,61 & \multirow{2}{*}{0,001} \\
\hline & Mitt. Rang & 48,11 & 29,33 & \\
\hline \multirow{2}{*}{ Zwanghaftigkeit } & $\mathrm{MW}$ & 1,31 & 0,81 & \multirow{2}{*}{0,013} \\
\hline & Mitt. Rang & 46,57 & 32,52 & \\
\hline \multirow{2}{*}{$\begin{array}{l}\text { Unsicherheit } \\
\text { Sozialkontakt }\end{array}$} & $\mathrm{MW}$ & 0,98 & 0,66 & \multirow{2}{*}{0,078} \\
\hline & Mitt. Rang & 45,22 & 35,31 & \\
\hline \multirow{2}{*}{ Depressivität } & $\mathrm{MW}$ & 1,44 & 0,87 & \multirow{2}{*}{0,004} \\
\hline & Mitt. Rang & 47,32 & 30,96 & \\
\hline \multirow{2}{*}{ Ängstlichkeit } & MW & 1,31 & 0,59 & \multirow{2}{*}{0,001} \\
\hline & Mitt. Rang & 47,92 & 29,72 & \\
\hline \multirow{2}{*}{ Aggressivität } & MW & 1,15 & 0,79 & \multirow{2}{*}{0,020} \\
\hline & Mitt. Rang & 46,24 & 33,20 & \\
\hline \multirow{2}{*}{ Phobische Angst } & MW & 0,60 & 0,17 & \multirow{2}{*}{0,009} \\
\hline & Mitt. Rang & 46,68 & 32,30 & \\
\hline \multirow{2}{*}{ Paranoides Denken } & MW & 1,08 & 0,67 & \multirow{2}{*}{0,013} \\
\hline & Mitt. Rang & 46,54 & 32,59 & \\
\hline \multirow{2}{*}{ Psychotizismus } & $\mathrm{MW}$ & 0,68 & 0,24 & \multirow{2}{*}{0,001} \\
\hline & Mitt. Rang & 47,90 & 29,76 & \\
\hline
\end{tabular}

Mann-Whitney-U-Test, 2-seitig

Tabelle 26: Lagemaße der Globalen Kennwerte des SCL 90-R nach Vernachlässigung

\begin{tabular}{|c|c|c|c|c|}
\hline $\begin{array}{l}\text { Globale Kennwerte nach } \\
\text { SCL } 90-R\end{array}$ & & $\begin{array}{l}\text { Personen mit } \\
\text { Missbrauchserf. } \\
(n=56)\end{array}$ & $\begin{array}{l}\text { Personen ohne } \\
\text { Missbrauchserf } \\
(n=27)\end{array}$ & p-Wert \\
\hline \multirow{2}{*}{ GSI } & $\mathrm{MW}$ & 1,12 & 0,63 & \multirow{2}{*}{0,002} \\
\hline & Mitt. Rang & 47,69 & 30,20 & \\
\hline \multirow{2}{*}{ PSDI } & MW & 2,05 & 1,73 & \multirow{2}{*}{0,027} \\
\hline & Mitt. Rang & 46,06 & 33,57 & \\
\hline \multirow{2}{*}{ PST } & MW & 45,98 & 29,29 & \multirow{2}{*}{0,001} \\
\hline & Mitt. Rang & 48,37 & 28,80 & \\
\hline
\end{tabular}


Beim Vergleich der vernachlässigten Personen nach Geschlecht zeigten sich wiederum kaum Unterschiede. Lediglich in den Skalenwerte zu phobischer Angst ( $p=0,037$ ) hatten die Frauen einen signifikant höheren Wert als die in der Kindheit vernachlässigten Männer.

Tabelle 27: Geschlechtsspezifische Unterschiede für die Composite Scores des EuropASI nach Vernachlässigung

\begin{tabular}{llll}
\hline $\begin{array}{l}\text { Composite Scores nach } \\
\text { EuropASI }\end{array}$ & $\chi$-Wert & $\begin{array}{l}\text { Freiheits- } \\
\text { grad }\end{array}$ & p-Wert \\
\hline Körperliche Probleme & 18,748 & 15 & 0,225 \\
& & & 0,331 \\
\hline Arbeit: Ökon. Situation & 5,751 & 5 & 0,735 \\
\hline Arbeit: Zufriedenheit & & 19 & 0,474 \\
\hline Alkoholgebrauch & 14,802 & 19 & 0,478 \\
\hline Drogengebrauch & 18,733 & 54 & 0,548 \\
\hline Rechtliche Probleme & 53,904 & 22 & 0,563 \\
\hline Familie & 20,561 & 30 & 0,459 \\
\hline Sozialbeziehungen & 28,150 & 18 & 0,395 \\
\hline Psychische Probleme & 17,957 & 36 & \\
\hline
\end{tabular}

Chi-Quadrat-Test, 2-seitig, nach Pearson

Tabelle 28: Geschlechtsspezifische Unterschiede für die Skalenwerten des SCL 90-R nach Vernachlässigung

\begin{tabular}{llll}
\hline $\begin{array}{l}\text { Skalenwerte nach } \\
\text { SCL 90-R }\end{array}$ & $\chi$-Wert & $\begin{array}{l}\text { Freiheits- } \\
\text { grad }\end{array}$ & p-Wert \\
\hline Somatisierung & 28,749 & 27 & 0,373 \\
\hline Zwanghaftigkeit & 28,190 & 24 & 0,252 \\
\hline Unsicherheit Sozialkontakt & 29,587 & 22 & 0,129 \\
\hline Depressivität & 31,544 & 32 & 0,490 \\
\hline Ängstlichkeit & 25,395 & 25 & 0,440 \\
\hline Aggressivität & 15,188 & 17 & 0,582 \\
\hline Phobische Angst & 24,717 & 14 & $\mathbf{0 , 0 3 7}$ \\
\hline
\end{tabular}




\begin{tabular}{llll}
\hline Paranoides Denken & 15,532 & 16 & 0,486 \\
\hline Psychotizismus & 22,250 & 18 & 0,221 \\
\hline Chi-Quadrat-Test, 2-seitig, nach Pearson & &
\end{tabular}

Tabelle 29: Geschlechtsspezifische Unterschiede für die Globalen Kennwerte des SCL 90R nach Vernachlässigung

\begin{tabular}{llll}
\hline $\begin{array}{l}\text { Globale Kennwerte nach } \\
\text { SCL 90-R }\end{array}$ & $\chi$-Wert & $\begin{array}{l}\text { Freiheits- } \\
\text { grad }\end{array}$ & p-Wert \\
\hline GSI & 49,012 & 46 & 0,353 \\
\hline PSDI & 56,000 & 54 & 0,400 \\
\hline PST & 40,627 & 38 & 0,355 \\
\hline
\end{tabular}

Chi-Quadrat-Test, 2-seitig, nach Pearson

\subsubsection{Familiäre Gewalt}

Unter familiärer Gewalt litt rund die Hälfte der Studienteilnehmer. Wie auch schon in den vorangegangenen Bereichen waren auch hier die Frauen wieder stärker betroffen, ohne dass das Ergebnis signifikant wurde. Die folgenden Auswertungen zeigen, ob sich die Gruppen mit und ohne familiärer Gewalt in der Kindheit bezüglich der Höhe ihrer Composite Scores und psychischer Symptombelastungen voneinander unterschieden.

Bei den Composite Scores des EuropASI stellten sich lediglich bei den psychischen Problemen signifikante Unterschiede zwischen Studienteilnehmern mit und ohne familiäre Gewalt in der Kindheit heraus.

Tabelle 30: Lagemaße der Composite Scores des EuropASI nach familiärer Gewalt

\begin{tabular}{lllll}
\hline $\begin{array}{l}\text { Composite Scores nach } \\
\text { EuropASI }\end{array}$ & & $\begin{array}{l}\text { Personen mit } \\
\text { Missbrauchserf. } \\
(\mathbf{n}=\mathbf{4 2})\end{array}$ & $\begin{array}{l}\text { Personen ohne } \\
\text { Missbrauchserf } \\
(\mathbf{n}=\mathbf{4 1})\end{array}$ & p-Wert \\
\hline \multirow{2}{*}{ Körperliche Probleme } & MW & 0,25 & 0,14 & \multirow{2}{*}{0,134} \\
\cline { 2 - 5 } & $\begin{array}{l}\text { Mitt. } \\
\text { Rang }\end{array}$ & 45,30 & 38,62 & \\
\hline
\end{tabular}




\begin{tabular}{|c|c|c|c|c|}
\hline \multirow{3}{*}{ Arbeit: Ökon. Situation } & MW & 0,86 & 0,71 & \multirow{3}{*}{0,196} \\
\hline & Mitt. & 44,62 & 39,32 & \\
\hline & \multicolumn{3}{|l|}{ Rang } & \\
\hline \multirow{3}{*}{ Arbeit: Zufriedenheit } & MW & 0,32 & 0,23 & \multirow{3}{*}{0,213} \\
\hline & Mitt. & 45,00 & 38,93 & \\
\hline & Rang & & & \\
\hline \multirow{3}{*}{ Alkoholgebrauch } & MW & 0,19 & 0,19 & \multirow{3}{*}{0,795} \\
\hline & Mitt. & 42,57 & 41,41 & \\
\hline & Rang & & & \\
\hline \multirow{3}{*}{ Drogengebrauch } & MW & 0,70 & 0,67 & \multirow{3}{*}{0,820} \\
\hline & Mitt. & 42,60 & 41,39 & \\
\hline & Rang & & & \\
\hline \multirow{3}{*}{ Rechtliche Probleme } & MW & 0,36 & 0,34 & \multirow{3}{*}{0,821} \\
\hline & Mitt. & 42,58 & 41,40 & \\
\hline & Rang & & & \\
\hline \multirow{3}{*}{ Familie } & $\mathrm{MW}$ & 0,32 & 0,23 & \multirow{3}{*}{0,213} \\
\hline & Mitt. & 45,13 & 38,79 & \\
\hline & Rang & & & \\
\hline \multirow{3}{*}{ Sozialbeziehungen } & MW & 0,19 & 0,12 & \multirow{3}{*}{0,194} \\
\hline & Mitt. & 45,05 & 38,88 & \\
\hline & Rang & & & \\
\hline \multirow{3}{*}{ Psychische Probleme } & $\mathrm{MW}$ & 0,37 & 0,24 & \multirow{3}{*}{0,010} \\
\hline & Mitt. & 48,74 & 35,10 & \\
\hline & Rang & & & \\
\hline
\end{tabular}

Mann-Whitney-U-Test, 2-seitig

Bei der Frage nach den Unterschieden in der Beurteilung der Skalenwerte zwischen Personen mit und ohne familiärer Gewalt in der Kindheit waren ein Großteil der aus dem SCL 90-R errechneten Größen signifikant geworden. Ausnahmen bildeten hier lediglich die Skalen Zwanghaftigkeit und Aggressivität. Diese Ergebnisse wiesen darauf hin, dass sich die Gruppen mit und ohne familiäre Missbrauchserfahrung in der psychischen Symptombelastung wesentlich unterscheiden. 
Die hier betrachtete Gruppe mit familiärer Missbrauchserfahrung wies außerdem hohe Mittelwerte in der psychischen Symptombelastung des SCL 90-R auf. Diese Mittelwerte waren allerdings im direkten Vergleich mit der Gruppe der sexuell misshandelten Patienten niedriger (siehe dazu Auswertungen in Tabelle 61 und Tabelle 62).

Tabelle 31: Lagemaße der Skalenwerte des SCL 90-R nach familiärer Gewalt

\begin{tabular}{|c|c|c|c|c|}
\hline $\begin{array}{l}\text { Skalenwerte nach } \\
\text { SCL 90-R }\end{array}$ & & $\begin{array}{l}\text { Personen mit } \\
\text { Missbrauchserf. } \\
(n=42)\end{array}$ & $\begin{array}{l}\text { Personen ohne } \\
\text { Missbrauchserf } \\
(n=41)\end{array}$ & p-Wert \\
\hline \multirow{2}{*}{ Somatisierung } & $\mathrm{MW}$ & 1,18 & 0,76 & \multirow{2}{*}{0,008} \\
\hline & Mitt. Rang & 48,94 & 34,89 & \\
\hline \multirow{2}{*}{ Zwanghaftigkeit } & $\mathrm{MW}$ & 1,31 & 0,99 & \multirow{2}{*}{0,078} \\
\hline & Mitt. Rang & 46,60 & 37,29 & \\
\hline \multirow{2}{*}{$\begin{array}{l}\text { Unsicherheit } \\
\text { Sozialkontakt }\end{array}$} & $\mathrm{MW}$ & 1,09 & 0,66 & \multirow{2}{*}{0,024} \\
\hline & Mitt. Rang & 47,89 & 35,96 & \\
\hline \multirow{2}{*}{ Depressivität } & MW & 1,52 & 0,99 & \multirow{2}{*}{0,004} \\
\hline & Mitt. Rang & 49,45 & 34,37 & \\
\hline \multirow{2}{*}{ Ängstlichkeit } & MW & 1,22 & 0,92 & \multirow{2}{*}{0,007} \\
\hline & Mitt. Rang & 49,04 & 34,79 & \\
\hline \multirow{2}{*}{ Aggressivität } & MW & 1,13 & 0,93 & \multirow{2}{*}{0,104} \\
\hline & Mitt. Rang & 46,23 & 37,67 & \\
\hline \multirow{2}{*}{ Phobische Angst } & MW & 0,65 & 0,27 & \multirow{2}{*}{0,009} \\
\hline & Mitt. Rang & 48,62 & 35,22 & \\
\hline \multirow{2}{*}{ Paranoides Denken } & MW & 1,19 & 0,70 & \multirow{2}{*}{0,010} \\
\hline & Mitt. Rang & 48,70 & 35,13 & \\
\hline \multirow{2}{*}{ Psychotizismus } & MW & 0,72 & 0,36 & \multirow{2}{*}{0,005} \\
\hline & Mitt. Rang & 49,29 & 34,54 & \\
\hline
\end{tabular}


Tabelle 32: Lagemaße der Globalen Kennwerte des SCL 90-R nach familiärer Gewalt

\begin{tabular}{lllll}
\hline $\begin{array}{l}\text { Globale Kennwerte nach } \\
\text { SCL 90-R }\end{array}$ & & $\begin{array}{l}\text { Personen mit } \\
\text { Missbrauchserf. } \\
(\mathbf{n = 4 2})\end{array}$ & $\begin{array}{l}\text { Personen ohne } \\
\text { Missbrauchserf } \\
(\mathbf{n}=\mathbf{4 1 )}\end{array}$ & p-Wert \\
\hline \multirow{2}{*}{ GSI } & MW & 1,16 & 0,76 & \multirow{2}{*}{$\mathbf{0 , 0 0 6}$} \\
\cline { 2 - 4 } & Mitt. Rang & 49,24 & 34,59 & \multirow{2}{*}{$\mathbf{0 , 0 4 9}$} \\
\hline \multirow{2}{*}{ PSDI } & MW & 2,05 & 1,84 & \multirow{2}{*}{$\mathbf{0 , 0 0 3}$} \\
\cline { 2 - 5 } & Mitt. Rang & 47,15 & 36,72 & 33,65 \\
\hline \multirow{2}{*}{ PST } & MW & 47,28 & 33,93 & \\
\cline { 2 - 5 } & Mitt. Rang & 49,88 & &
\end{tabular}

Mann-Whitney-U-Test, 2-seitig

Geschlechtsspezifische Unterschiede fanden sich bei den emotional missbrauchten Personen nicht (vgl. die folgenden Tabellen).

Tabelle 33: Geschlechtsspezifische Unterschiede für die Composite Scores des EuropASI nach familiärer Gewalt

\begin{tabular}{llll}
\hline $\begin{array}{l}\text { Composite Scores nach } \\
\text { EuropASI }\end{array}$ & $\chi$-Wert & $\begin{array}{l}\text { Freiheits- } \\
\text { grad }\end{array}$ & p-Wert \\
\hline Körperliche Probleme & 13,335 & 11 & 0,272 \\
\hline Arbeit: Ökon. Situation & 4,640 & 5 & 0,461 \\
& & & \\
\hline Arbeit: Zufriedenheit & 14,772 & 16 & 0,541 \\
\hline Alkoholgebrauch & 13,417 & 14 & 0,494 \\
\hline Drogengebrauch & 39,880 & 40 & 0,476 \\
\hline Rechtliche Probleme & 22,661 & 20 & 0,306 \\
\hline Familie & 24,049 & 25 & 0,517 \\
\hline Sozialbeziehungen & 12,510 & 13 & 0,486 \\
\hline Psychische Probleme & 24,332 & 28 & 0,664 \\
\hline
\end{tabular}

Chi-Quadrat-Test, 2-seitig, nach Pearson 
Tabelle 34: Geschlechtsspezifische Unterschiede für die Skalenwerten des SCL 90-R EuropASI nach familiärer Gewalt

\begin{tabular}{llll}
\hline $\begin{array}{l}\text { Skalenwerte nach } \\
\text { SCL 90-R }\end{array}$ & $\chi$-Wert & $\begin{array}{l}\text { Freiheits- } \\
\text { grad }\end{array}$ & p-Wert \\
\hline Somatisierung & 29,844 & 26 & 0,274 \\
\hline Zwanghaftigkeit & 26,805 & 23 & 0,264 \\
\hline Unsicherheit Sozialkontakt & 28,925 & 21 & 0,116 \\
\hline Depressivität & 29,986 & 28 & 0,364 \\
\hline Ängstlichkeit & 18,112 & 20 & 0,580 \\
\hline Aggressivität & 15,154 & 15 & 0,440 \\
\hline Phobische Angst & 19,118 & 14 & 0,160 \\
\hline Paranoides Denken & 12,671 & 15 & 0,628 \\
& & & \\
\hline Psychotizismus & 22,212 & 16 & 0,136 \\
\hline Chi-Quadrat-Test, 2-seitig, nach Pearson & & &
\end{tabular}

Chi-Quadrat-Test, 2-seitig, nach Pearson

Tabelle 35: Geschlechtsspezifische Unterschiede für die Globalen Kennwerte des SCL 90R EuropASI nach familiärer Gewalt

\begin{tabular}{llll}
\hline $\begin{array}{l}\text { Globale Kennwerte nach } \\
\text { SCL 90-R }\end{array}$ & $\chi$-Wert & $\begin{array}{l}\text { Freiheits- } \\
\text { grad }\end{array}$ & p-Wert \\
\hline GSI & 37,760 & 35 & 0,344 \\
\hline PSDI & 42,000 & 40 & 0,384 \\
& & & \\
\hline PST & 34,933 & 32 & 0,330 \\
\hline
\end{tabular}

Chi-Quadrat-Test, 2-seitig, nach Pearson

\subsubsection{Emotionale Misshandlung}

Von emotionaler Misshandlung in der Kindheit waren rund zwei Drittel aller Befragten betroffen. Hier waren Männer und Frauen in etwa gleich häufig belastet. Nach Trennung der Datei in zwei Gruppen, mit und ohne emotionale Misshandlung, zeigten sich die folgenden Ergebnisse: Signifikante Unterschiede beim Composite Score fanden sich in den Bereichen Arbeit und Unterhalt: 
Ökonomische Situation, Sozialbeziehungen und psychische Probleme mit einer stärkeren Belastung der Personen mit Missbrauchserfahrungen.

Tabelle 36: Lagemaße der Composite Scores des EuropASI nach emotionaler Missbrauchserfahrung

\begin{tabular}{|c|c|c|c|c|}
\hline $\begin{array}{l}\text { Composite Scores nach } \\
\text { EuropASI }\end{array}$ & & $\begin{array}{l}\text { Personen mit } \\
\text { Missbrauchserf. } \\
(n=55)\end{array}$ & $\begin{array}{l}\text { Personen ohne } \\
\text { Missbrauchserf } \\
(n=28)\end{array}$ & p-Wert \\
\hline & $\mathrm{MW}$ & 0,24 & 0,11 & \\
\hline Körperliche Probleme & $\begin{array}{l}\text { Mitt. } \\
\text { Rang }\end{array}$ & 44,67 & 36,46 & 0,084 \\
\hline & MW & 0,84 & 0,66 & \\
\hline Arbeit: Ökon. Situation & $\begin{array}{l}\text { Mitt. } \\
\text { Rang }\end{array}$ & 44,83 & 36,13 & 0,047 \\
\hline & MW & 0,30 & 0,24 & \\
\hline Arbeit: Zufriedenheit & $\begin{array}{l}\text { Mitt. } \\
\text { Rang }\end{array}$ & 43,88 & 38,11 & 0,268 \\
\hline Alkoholgebrauch & $\begin{array}{l}\text { MW } \\
\text { Mitt. } \\
\text { Rang }\end{array}$ & $\begin{array}{l}0,16 \\
40,82\end{array}$ & $\begin{array}{l}0,25 \\
44,44\end{array}$ & 0,446 \\
\hline Drogengebrauch & $\begin{array}{l}\text { MW } \\
\text { Mitt. } \\
\text { Rang }\end{array}$ & $\begin{array}{l}0,68 \\
40,86\end{array}$ & $\begin{array}{l}0,70 \\
44,37\end{array}$ & 0,534 \\
\hline Rechtliche Probleme & $\begin{array}{l}\text { MW } \\
\text { Mitt. } \\
\text { Rang }\end{array}$ & $\begin{array}{l}0,33 \\
40,69\end{array}$ & $\begin{array}{l}0,38 \\
44,72\end{array}$ & 0,470 \\
\hline Familie & $\begin{array}{l}\text { MW } \\
\text { Mitt. } \\
\text { Rang }\end{array}$ & $\begin{array}{l}0,32 \\
44,33\end{array}$ & $\begin{array}{l}0,20 \\
37,17\end{array}$ & 0,187 \\
\hline Sozialbeziehungen & $\begin{array}{l}\text { MW } \\
\text { Mitt. } \\
\text { Rang }\end{array}$ & $\begin{array}{l}0,19 \\
45,24\end{array}$ & $\begin{array}{l}0,08 \\
35,28\end{array}$ & 0,049 \\
\hline Psychische Probleme & $\begin{array}{l}\text { MW } \\
\text { Mitt. } \\
\text { Rang }\end{array}$ & $\begin{array}{l}0,34 \\
45,62\end{array}$ & $\begin{array}{l}0,23 \\
34,50\end{array}$ & 0,049 \\
\hline
\end{tabular}


Die Skalenwerte des SCL 90-R lieferten eine nachweisbare Signifikanz in den Skalen Somatisierung, Depressivität, Ängstlichkeit und Psychotizismus. Die globalen Kennwerte des SCL 90-R waren allesamt signifikant.

Tabelle 37: Lagemaße der Skalenwerte des SCL 90-R nach emotionaler Missbrauchserfahrung

\begin{tabular}{|c|c|c|c|c|}
\hline $\begin{array}{l}\text { Skalenwerte nach } \\
\text { SCL 90-R }\end{array}$ & & $\begin{array}{l}\text { Personen mit } \\
\text { Missbrauchserf. } \\
(n=55)\end{array}$ & $\begin{array}{l}\text { Personen ohne } \\
\text { Missbrauchserf } \\
(n=28)\end{array}$ & p-Wert \\
\hline \multirow{2}{*}{ Somatisierung } & $\mathrm{MW}$ & 1,11 & 0,69 & \multirow{2}{*}{0,014} \\
\hline & Mitt. Rang & 46,52 & 32,63 & \\
\hline \multirow{2}{*}{ Zwanghaftigkeit } & MW & 1,22 & 1,00 & \multirow{2}{*}{0,386} \\
\hline & Mitt. Rang & 43,59 & 38,70 & \\
\hline \multirow{2}{*}{$\begin{array}{l}\text { Unsicherheit } \\
\text { Sozialkontakt }\end{array}$} & MW & 0,98 & 0,66 & \multirow{2}{*}{0,281} \\
\hline & Mitt. Rang & 43,97 & 37,91 & \\
\hline \multirow{2}{*}{ Depressivität } & MW & 1,40 & 0,96 & \multirow{2}{*}{0,031} \\
\hline & Mitt. Rang & 45,96 & 33,80 & \\
\hline \multirow{2}{*}{ Ängstlichkeit } & MW & 1,22 & 0,76 & \multirow{2}{*}{0,038} \\
\hline & Mitt. Rang & 45,81 & 34,09 & \\
\hline \multirow{2}{*}{ Aggressivität } & MW & 1,10 & 0,90 & \multirow{2}{*}{0,249} \\
\hline & Mitt. Rang & 44,11 & 37,63 & \\
\hline \multirow{2}{*}{ Phobische Angst } & MW & 0,56 & 0,27 & \multirow{2}{*}{0,072} \\
\hline & Mitt. Rang & 45,21 & 35,33 & \\
\hline \multirow{2}{*}{ Paranoides Denken } & MW & 1,09 & 0,66 & \multirow{2}{*}{0,094} \\
\hline & Mitt. Rang & 45,06 & 35,65 & \\
\hline \multirow{2}{*}{ Psychotizismus } & MW & 0,69 & 0,24 & \multirow{2}{*}{0,003} \\
\hline & Mitt. Rang & 47,43 & 30,74 & \\
\hline
\end{tabular}

Mann-Whitney-U-Test, 2-seitig 
Tabelle 38: Lagemaße der Globalen Kennwerte des SCL 90-R nach emotionaler Missbrauchserfahrung

\begin{tabular}{lllll}
\hline $\begin{array}{l}\text { Globale Kennwerte nach } \\
\text { SCL 90-R }\end{array}$ & & $\begin{array}{l}\text { Personen mit } \\
\text { Missbrauchserf. } \\
(\mathbf{n = 5 5 )}\end{array}$ & $\begin{array}{l}\text { Personen ohne } \\
\text { Missbrauchserf } \\
(\mathbf{n = 2 8})\end{array}$ & p-Wert \\
\hline \multirow{2}{*}{ GSI } & MW & 1,08 & 0,72 & $\mathbf{0 , 0 2 8}$ \\
\cline { 2 - 4 } & Mitt. Rang & 46,04 & 33,61 & \multirow{2}{*}{$\mathbf{0 , 0 4 4}$} \\
\hline \multirow{2}{*}{ PSDI } & MW & 2,04 & 1,74 & \multirow{2}{*}{$\mathbf{0 , 0 3 7}$} \\
\cline { 2 - 5 } & Mitt. Rang & 45,70 & 34,33 & \\
\hline \multirow{2}{*}{ PST } & MW & 43,89 & 33,62 & 34,07 \\
\hline
\end{tabular}

Mann-Whitney-U-Test, 2-seitig

Hinsichtlich der geschlechtsspezifischen Unterschiede bei Vorliegen von emotionaler Misshandlung, fanden sich bei den Skalen Unsicherheit im Sozialkontakt $(p=0,032)$ und phobische Angst $(p=0,050)$ signifikant höhere Werte bei den Frauen.

Tabelle 39: Geschlechtsspezifische Unterschiede für die Composite Scores des EuropASI nach emotionaler Misshandlung

\begin{tabular}{llll}
\hline $\begin{array}{l}\text { Composite Scores nach } \\
\text { EuropASI }\end{array}$ & $\chi$-Wert & $\begin{array}{l}\text { Freiheits- } \\
\text { grad }\end{array}$ & p-Wert \\
\hline Körperliche Probleme & 20,173 & 13 & 0,091 \\
\hline Arbeit: Ökon. Situation & 5,227 & 5 & 0,389 \\
\hline Arbeit: Zufriedenheit & 14,399 & 18 & 0,703 \\
\hline Alkoholgebrauch & 17,488 & 17 & 0,422 \\
\hline Drogengebrauch & & & 0,481 \\
\hline Rechtliche Probleme & 53,822 & 54 & 0,638 \\
\hline Familie & & & 0,493 \\
\hline
\end{tabular}




\begin{tabular}{lccc}
\hline & & & \\
\hline Sozialbeziehungen & 16,825 & 17 & 0,466 \\
& & & \\
\hline Psychische Probleme & 35,964 & 34 & 0,377 \\
& & & \\
\hline Chi-Quadrat-Test, 2-seitig, nach Pearson & & &
\end{tabular}

Tabelle 40: Geschlechtsspezifische Unterschiede für die Skalenwerten des SCL 90-R nach emotionaler Misshandlung 
Tabelle 41: Geschlechtsspezifische Unterschiede für die Globalen Kennwerte des SCL 90R nach emotionaler Misshandlung

\begin{tabular}{llll}
\hline $\begin{array}{l}\text { Globale Kennwerte nach } \\
\text { SCL 90-R }\end{array}$ & $\chi$-Wert & $\begin{array}{l}\text { Freiheits- } \\
\text { grad }\end{array}$ & p-Wert \\
\hline GSI & 48,741 & 45 & 0,325 \\
& & & 0,400 \\
\hline PSDI & 56,000 & 54 & 0,494 \\
\hline PST & & & \\
\hline Chi-Quadrat-Test, 2-seitig, nach Pearson & 41,481 & 42 & \\
\hline
\end{tabular}

\subsubsection{Schweregrad}

\subsubsection{Schweregrad allgemeiner Missbrauch}

Der Schweregrad (SG) des Missbrauchs wurde, wie in Kapitel 3.1.4 beschrieben, ermittelt und in die Kategorien „leicht“, „mittelschwer“ und „schwer“ unterteilt.

Bei der Frage nach allgemeinem Missbrauch zeigte sich, dass in 16 Fällen $(19,3 \%)$ kein Missbrauch, in 2 Fällen (2,4\%) leichter, bei 6 Personen $(7,2 \%)$ mittelschwerer und bei 59 Befragten (71,1\%) schwerer Missbrauch vorlag. Von allen Befragten mit schwerem Missbrauch waren die Männer mit rund 63\% am stärksten betroffen. In den beiden anderen Kategorien war das Geschlechtsverhältnis ausgeglichen. 78,6\% aller Frauen hatte irgendeine Art des Missbrauchs in der Kindheit erlebt. Bei den Männern betrug dieser Anteil rund $67 \%$.

Anschließend wurde untersucht, ob sich diese Schweregradeinteilungen hinsichtlich der Höhe der Skalenwerte voneinander unterscheiden. Aufgrund der geringen Fallzahlen in den Kategorien leichter und mittlerer Schweregrad wurden diese beiden zusammengefasst und der Kategorie schwerer Missbrauch gegenübergestellt. Die Ergebnisse sind in Tabelle 42, Tabelle 43 und Tabelle 44 
dargestellt. Der EuropASI blieb bezüglich der Unterscheidung des Schweregrads ohne signifikante Ergebnisse. Eine Unterscheidung des Schweregrads bot keine weiteren Erkenntnisse.

Tabelle 42: Einfluss des Schweregrads des allgemeinen Missbrauchs auf die Composite Scores des EuropASI

\begin{tabular}{|c|c|c|c|}
\hline $\begin{array}{l}\text { Composite Scores nach } \\
\text { EuropASI }\end{array}$ & Schweregrad & $\begin{array}{l}\text { Mittlerer } \\
\text { Rang }\end{array}$ & p-Wert \\
\hline \multirow{2}{*}{ Körperliche Probleme } & Leicht \& Mittel & 29,25 & \multirow{2}{*}{0,397} \\
\hline & Schwer & 34,64 & \\
\hline \multirow{2}{*}{ Arbeit: Ökon. Situation } & Leicht \& Mittel & 33,38 & \multirow{2}{*}{0,899} \\
\hline & Schwer & 34,08 & \\
\hline \multirow{2}{*}{ Arbeit: Zufriedenheit } & Leicht \& Mittel & 32,88 & \multirow{2}{*}{0,853} \\
\hline & Schwer & 34,15 & \\
\hline \multirow{2}{*}{ Alkoholgebrauch } & Leicht \& Mittel & 38,50 & \multirow{2}{*}{0,411} \\
\hline & Schwer & 33,39 & \\
\hline \multirow{2}{*}{ Drogengebrauch } & Leicht \& Mittel & 23,38 & \multirow{2}{*}{0,100} \\
\hline & Schwer & 35,44 & \\
\hline \multirow{2}{*}{ Rechtliche Probleme } & Leicht \& Mittel & 34,38 & \multirow{2}{*}{0,953} \\
\hline & Schwer & 33,95 & \\
\hline \multirow{2}{*}{ Familie } & Leicht \& Mittel & 33,75 & \multirow{2}{*}{0,968} \\
\hline & Schwer & 34,03 & \\
\hline \multirow{2}{*}{ Sozialbeziehungen } & Leicht \& Mittel & 23,50 & \multirow{2}{*}{0,077} \\
\hline & Schwer & 35,42 & \\
\hline \multirow{2}{*}{ Psychische Probleme } & Leicht \& Mittel & 27,44 & \multirow{2}{*}{0,310} \\
\hline & Schwer & 34,89 & \\
\hline
\end{tabular}

Mann-Whitney-Test, 2-seitig

Hingegen fanden sich bei mehreren Parametern des SCL 90-R hinsichtlich der Unterscheidung nach dem Schweregrad des Missbrauchs signifikante Unterschiede. Die Patienten zeigten bezüglich Depressivität, Ängstlichkeit, Aggressivität, Psychotizismus sowie den globalen Kennwerten GSI und PST signifikant schlechtere Werte bei einem schweren Missbrauch als bei einem 
leichten und mittelschweren Missbrauch. Eine Einteilung nach Schweregrad in leicht \& mittelschwer sowie schwer ist hier sinnvoll.

Tabelle 43: Einfluss des Schweregrads des allgemeinen Missbrauchs auf die Skalenwerte des SCL 90-R

\begin{tabular}{|c|c|c|c|}
\hline $\begin{array}{l}\text { Skalenwerte nach } \\
\text { SCL 90-R }\end{array}$ & Schweregrad & $\begin{array}{l}\text { Mittlerer } \\
\text { Rang }\end{array}$ & p-Wert \\
\hline \multirow{2}{*}{ Somatisierung } & Leicht \& Mittel & 21,88 & \multirow{2}{*}{0,060} \\
\hline & Schwer & 35,64 & \\
\hline \multirow{2}{*}{ Zwanghaftigkeit } & Leicht \& Mittel & 21,50 & \multirow{2}{*}{0,053} \\
\hline & Schwer & 35,69 & \\
\hline \multirow{2}{*}{ Unsicherheit Sozialkontakt } & Leicht \& Mittel & 23,94 & \multirow{2}{*}{0,119} \\
\hline & Schwer & 35,36 & \\
\hline \multirow{2}{*}{ Depressivität } & Leicht \& Mittel & 17,06 & \multirow{2}{*}{0,009} \\
\hline & Schwer & 36,30 & \\
\hline \multirow{2}{*}{ Ängstlichkeit } & Leicht \& Mittel & 20,56 & \multirow{2}{*}{0,037} \\
\hline & Schwer & 35,82 & \\
\hline \multirow{2}{*}{ Aggressivität } & Leicht \& Mittel & 20,88 & \multirow{2}{*}{0,041} \\
\hline & Schwer & 35,78 & \\
\hline \multirow{2}{*}{ Phobische Angst } & Leicht \& Mittel & 23,06 & \multirow{2}{*}{0,084} \\
\hline & Schwer & 35,48 & \\
\hline \multirow{2}{*}{ Paranoides Denken } & Leicht \& Mittel & 22,38 & \multirow{2}{*}{0,071} \\
\hline & Schwer & 35,58 & \\
\hline \multirow{2}{*}{ Psychotizismus } & Leicht \& Mittel & 20,44 & \multirow{2}{*}{0,034} \\
\hline & Schwer & 35,84 & \\
\hline
\end{tabular}

Mann-Whitney-Test, 2-seitig

Tabelle 44: Einfluss des Schweregrads des allgemeinen Missbrauchs auf die Globalen Kennwerte des SCL 90-R

\begin{tabular}{llll}
\hline $\begin{array}{l}\text { Globale Kennwerte nach } \\
\text { SCL 90-R }\end{array}$ & Schweregrad & $\begin{array}{l}\text { Mittlerer } \\
\text { Rang }\end{array}$ & p-Wert \\
\hline \multirow{2}{*}{ GSI } & Leicht \& Mittel & 20,19 & \multirow{2}{*}{$\mathbf{0 , 0 3 3}$} \\
\cline { 2 - 3 } & Schwer & 35,87 & \\
\hline PSDI & Leicht \& Mittel & 22,38 & 0,072 \\
\hline
\end{tabular}




\begin{tabular}{|c|c|c|c|}
\hline & Schwer & 35,58 & \\
\hline \multirow{2}{*}{ PST } & Leicht \& Mittel & 20,44 & \multirow{2}{*}{0,036} \\
\hline & Schwer & 35,84 & \\
\hline
\end{tabular}

Mann-Whitney-Test, 2-seitig

\subsubsection{Schweregrad sexueller Missbrauch}

Bei dieser Fragestellung trat die Kategorie „leicht“ kein einziges Mal ausschließlich auf. Zwar wurde ein leichter Missbrauch von den Patienten des öfteren angegeben, aber immer in Zusammenhang mit einem mittelschweren oder schweren Missbrauch. Auf Grund der Bewertungsregeln wurde in einem solchen Fall der höchste auftretende Schweregrad gezählt. Damit gaben 15,7\% einen mittelschweren und 14,5\% einen schweren Grad an sexuellem Missbrauch an. Knapp $70 \%$ der Befragten gaben keinen derartigen Missbrauch in der Kindheit an. Von den Frauen fielen 28,6\% in die mittelschwerere Kategorie und $25 \%$ in die schwere Kategorie von sexuellem Missbrauch. Bei den Männern waren dies für diese beiden Kategorien jeweils 9,1\%. Männer und Frauen unterscheiden sich damit signifikant in der Häufigkeit des Auftretens von mittelschweren und schweren sexuellen Missbrauch ( $\chi$-Wert 11,069, Freiheitsgrad 2, P-Wert 0,004, Chi-Quadrat nach Pearson, 2-seitig).

Beim EuropASI war lediglich der Problembereich körperliche Probleme für die Einstufung der Schweregrade signifikant.

Tabelle 45: Einfluss des Schweregrads des sexuellen Missbrauchs auf die Composite Scores des EuropASI

\begin{tabular}{|c|c|c|c|}
\hline $\begin{array}{lll}\text { Composite } & \text { Scores } & \text { nach } \\
\text { EuropASI } & & \end{array}$ & Schweregrad & $\begin{array}{l}\text { Mittlerer } \\
\text { Rang }\end{array}$ & p-Wert \\
\hline \multirow{2}{*}{ Körperliche Probleme } & Leicht \& Mittel & 9,69 & \multirow{2}{*}{0,010} \\
\hline & Schwer & 16,58 & \\
\hline \multirow{2}{*}{ Arbeit: Ökon. Situation } & Leicht \& Mittel & 12,42 & \multirow{2}{*}{0,619} \\
\hline & Schwer & 13,63 & \\
\hline
\end{tabular}




\begin{tabular}{|c|c|c|c|}
\hline \multirow{2}{*}{ Arbeit: Zufriedenheit } & Leicht \& Mittel & 14,08 & \multirow{2}{*}{0,411} \\
\hline & Schwer & 11,83 & \\
\hline \multirow{2}{*}{ Alkoholgebrauch } & Leicht \& Mittel & 13,23 & \multirow{2}{*}{0,849} \\
\hline & Schwer & 12,75 & \\
\hline \multirow{2}{*}{ Drogengebrauch } & Leicht \& Mittel & 12,54 & \multirow{2}{*}{0,744} \\
\hline & Schwer & 13,50 & \\
\hline \multirow{2}{*}{ Rechtliche Probleme } & Leicht \& Mittel & 12,35 & \multirow{2}{*}{0,628} \\
\hline & Schwer & 13,71 & \\
\hline \multirow{2}{*}{ Familie } & Leicht \& Mittel & 13,58 & \multirow{2}{*}{0,665} \\
\hline & Schwer & 12,38 & \\
\hline \multirow{2}{*}{ Sozialbeziehungen } & Leicht \& Mittel & 11,19 & \multirow{2}{*}{0,159} \\
\hline & Schwer & 14,96 & \\
\hline \multirow{2}{*}{ Psychische Probleme } & Leicht \& Mittel & 14,08 & \multirow{2}{*}{0,446} \\
\hline & Schwer & 11,83 & \\
\hline
\end{tabular}

Mann-Whitney-Test, 2-seitig

Beim SCL 90-R zeigten sich deutliche Unterschiede in den Schweregradkategorien des sexuellen Missbrauchs. Bis auf die Skalenwerte Ängstlichkeit und Psychotizismus waren alle anderen Werte signifikant. Dies weist auf eine erheblich stärkere psychische Symptombelastung derjenigen Patienten hin, die in die höchste Schweregrad-Kategorie des sexuellen Missbrauchs fallen. Eine Unterscheidung des Schweregrads in leicht \& mittel sowie schwer bietet sich an.

Tabelle 46: Einfluss des Schweregrads des sexuellen Missbrauchs auf die Skalenwerte des SCL 90-R

\begin{tabular}{llll}
\hline $\begin{array}{l}\text { Skalenwerte nach } \\
\text { SCL 90-R }\end{array}$ & Schweregrad & $\begin{array}{l}\text { Mittlerer } \\
\text { Rang }\end{array}$ & p-Wert \\
\hline \multirow{2}{*}{ Somatisierung } & Leicht \& Mittel & 9,35 & \multirow{2}{*}{$\mathbf{0 , 0 1 0}$} \\
\cline { 2 - 3 } & Schwer & 16,96 & \\
\hline \multirow{2}{*}{ Zwanghaftigkeit } & Leicht \& Mittel & 9,81 & \multirow{2}{*}{$\mathbf{0 2 3}$} \\
\cline { 2 - 3 } & Schwer & 16,46 & \multirow{2}{*}{$\mathbf{0 , 0 2 0}$} \\
\hline \multirow{2}{*}{ Unsicherheit Sozialkontakt } & Leicht \& Mittel & 9,73 & \\
\cline { 2 - 3 } & Schwer & 16,54 & \\
\hline
\end{tabular}




\begin{tabular}{|c|c|c|c|}
\hline \multirow{2}{*}{ Depressivität } & Leicht \& Mittel & 9,31 & \multirow{2}{*}{0,009} \\
\hline & Schwer & 17,00 & \\
\hline \multirow{2}{*}{ Ängstlichkeit } & Leicht \& Mittel & 10,46 & \multirow{2}{*}{0,072} \\
\hline & Schwer & 15,75 & \\
\hline \multirow{2}{*}{ Aggressivität } & Leicht \& Mittel & 9,73 & \multirow{2}{*}{0,020} \\
\hline & Schwer & 16,54 & \\
\hline \multirow{2}{*}{ Phobische Angst } & Leicht \& Mittel & 9,62 & \multirow{2}{*}{0,015} \\
\hline & Schwer & 16,67 & \\
\hline \multirow{2}{*}{ Paranoides Denken } & Leicht \& Mittel & 10,23 & \multirow{2}{*}{0,049} \\
\hline & Schwer & 16,00 & \\
\hline \multirow{2}{*}{ Psychotizismus } & Leicht \& Mittel & 10,38 & \multirow{2}{*}{0,063} \\
\hline & Schwer & 15,83 & \\
\hline
\end{tabular}

Tabelle 47: Einfluss des Schweregrads des sexuellen Missbrauchs auf die Globalen Kennwerte des SCL 90-R

\begin{tabular}{llll}
\hline $\begin{array}{lll}\text { Globale Kennwerte nach } \\
\text { SCL 90-R }\end{array}$ & Schweregrad & $\begin{array}{l}\text { Mittlerer } \\
\text { Rang }\end{array}$ & p-Wert \\
\hline \multirow{2}{*}{ GSI } & Leicht \& Mittel & 9,50 & $\mathbf{0 , 0 1 3}$ \\
\cline { 2 - 3 } & Schwer & 16,79 & \\
\hline \multirow{2}{*}{ PSDI } & Leicht \& Mittel & 9,54 & \multirow{2}{*}{$\mathbf{0 , 0 1 4}$} \\
\cline { 2 - 3 } & Schwer & 16,75 & \multirow{2}{*}{$\mathbf{0 , 0 0 7}$} \\
\hline \multirow{2}{*}{ PST } & Leicht \& Mittel & 9,19 & \\
\cline { 2 - 3 } & Schwer & 17,13 & \\
\hline
\end{tabular}

Mann-Whitney-Test, 2-seitig

\subsubsection{Schweregrad körperlicher Gewalt}

Ein leichter Schweregrad von körperlichem Missbrauch wurde bei 8,4\%, ein mittelschwerer bei $14,5 \%$ der Befragten festgestellt und schwerer körperlicher Missbrauch fand sich bei 31,3\% der Personen. 45,8\% der Befragten gaben keine derartigen Missbrauchserlebnisse in der Kindheit an. Bei schwerem körperlichem Missbrauch waren Männer zu fast $70 \%$ betroffen, in der mittleren und auch annähernd in der leichten Schweregradkategorie war das Geschlechtsverhältnis 
ausgeglichen. Insgesamt waren rund ein Drittel der Männer und 29\% der Frauen von schwerem körperlichen Missbrauch in der Kindheit betroffen. Männer und Frauen unterscheiden sich damit nicht signifikant $(\chi$-Wert 2,173, Freiheitsgrad 3 , P-Wert 0,537, Chi-Quadrat nach Pearson, 2-seitig).

Wie aus der folgenden Tabelle erkennbar ist, zeigten Patienten mit körperlicher Missbrauchserfahrung bei körperlichen Problemen, Sozialbeziehungen und psychischen Problemen signifikante Unterschiede in Abhängig vom Schweregrad des Missbrauchs. Bei genauerer Betrachtung erkennt man, dass die Belastungswerte bei keinem vorliegenden Missbrauch und leichten Missbrauch zusammenfallen, ebenso bei einem mittelschweren und schwereren Missbrauch.

Tabelle 48: Einfluss des Schweregrads der körperlichen Gewalt auf die Composite Scores des EuropASI

\begin{tabular}{|c|c|c|c|c|c|}
\hline $\begin{array}{lll}\text { Composite } & \text { Scores } & \text { nach } \\
\text { EuropASI } & & \end{array}$ & Schweregrad & $\begin{array}{l}\text { Mittlerer } \\
\text { Rang }\end{array}$ & $\chi$-Wert & df & p-Wert \\
\hline \multirow{4}{*}{ Körperliche Probleme } & Keine & 35,71 & \multirow{4}{*}{10,702} & \multirow{4}{*}{3} & \multirow{4}{*}{0,013} \\
\hline & Leicht & 33,29 & & & \\
\hline & Mittel & 50,42 & & & \\
\hline & Schwer & 49,65 & & & \\
\hline \multirow{4}{*}{ Arbeit: Ökon. Situation } & Keine & 39,26 & \multirow{4}{*}{5,057} & \multirow{4}{*}{3} & \multirow{4}{*}{0,168} \\
\hline & Leicht & 41,00 & & & \\
\hline & Mittel & 53,00 & & & \\
\hline & Schwer & 41,19 & & & \\
\hline \multirow{4}{*}{ Arbeit: Zufriedenheit } & Keine & 40,67 & \multirow{4}{*}{2,139} & \multirow{4}{*}{3} & \multirow{4}{*}{0,544} \\
\hline & Leicht & 48,71 & & & \\
\hline & Mittel & 48,29 & & & \\
\hline & Schwer & 39,23 & & & \\
\hline \multirow{4}{*}{ Alkoholgebrauch } & Keine & 39,66 & \multirow{4}{*}{5,110} & \multirow{4}{*}{3} & \multirow{4}{*}{0,164} \\
\hline & Leicht & 33,43 & & & \\
\hline & Mittel & 52,67 & & & \\
\hline & Schwer & 42,81 & & & \\
\hline Drogengebrauch & Keine & 39,50 & 1,157 & 3 & 0,763 \\
\hline
\end{tabular}




\begin{tabular}{|c|c|c|c|c|c|}
\hline & Leicht & 45,71 & & & \\
\hline & Mittel & 40,33 & & & \\
\hline & Schwer & 45,42 & & & \\
\hline \multirow{4}{*}{ Rechtliche Probleme } & Keine & 39,00 & \multirow{4}{*}{1,553} & \multirow{4}{*}{3} & \multirow{4}{*}{0,670} \\
\hline & Leicht & 50,00 & & & \\
\hline & Mittel & 43,96 & & & \\
\hline & Schwer & 43,33 & & & \\
\hline \multirow{4}{*}{ Familie } & Keine & 37,03 & \multirow{4}{*}{5,731} & \multirow{4}{*}{3} & \multirow{4}{*}{0,125} \\
\hline & Leicht & 33,50 & & & \\
\hline & Mittel & 49,25 & & & \\
\hline & Schwer & 48,21 & & & \\
\hline \multirow{4}{*}{ Sozialbeziehungen } & Keine & 34,86 & \multirow{4}{*}{9,132} & \multirow{4}{*}{3} & \multirow{4}{*}{0,028} \\
\hline & Leicht & 39,14 & & & \\
\hline & Mittel & 48,08 & & & \\
\hline & Schwer & 50,40 & & & \\
\hline \multirow{4}{*}{ Psychische Probleme } & Keine & 34,21 & \multirow{4}{*}{14,324} & \multirow{4}{*}{3} & \multirow{4}{*}{0,002} \\
\hline & Leicht & 26,50 & & & \\
\hline & Mittel & 52,54 & & & \\
\hline & Schwer & 52,69 & & & \\
\hline
\end{tabular}

Kruskal-Wallis-Test

Die Ergebnisse in Tabelle 49 und Tabelle 50 zeigen auf, dass in den Skalenwerten und globalen Kennwerten des SCL 90-R keine Unterschiede in den Schweregradkategorien für den körperlichen Missbrauch vorlagen.

Tabelle 49: Einfluss des Schweregrads der körperlichen Gewalt auf die Skalenwerte des SCL 90-R

\begin{tabular}{|c|c|c|c|c|c|}
\hline $\begin{array}{l}\text { Skalenwerte nach } \\
\text { SCL } 90-R\end{array}$ & Schweregrad & $\begin{array}{l}\text { Mittlerer } \\
\text { Rang }\end{array}$ & $\chi$-Wert & $\overline{d f}$ & p-Wert \\
\hline \multirow{4}{*}{ Somatisierung } & Keine & 37,41 & \multirow{4}{*}{5,719} & \multirow{4}{*}{3} & \multirow{4}{*}{0,126} \\
\hline & Leicht & 49,93 & & & \\
\hline & Mittel & 35,29 & & & \\
\hline & Schwer & 49,67 & & & \\
\hline \multirow[t]{2}{*}{ Zwanghaftigkeit } & Keine & 40,37 & \multirow[t]{2}{*}{7,010} & \multirow[t]{2}{*}{3} & \multirow[t]{2}{*}{0,072} \\
\hline & Leicht & 29,07 & & & \\
\hline
\end{tabular}




\begin{tabular}{|c|c|c|c|c|c|}
\hline & Mittel & 34,88 & & & \\
\hline & Schwer & 51,15 & & & \\
\hline \multirow{4}{*}{ Unsicherheit Sozialkontakt } & Keine & 38,86 & \multirow{4}{*}{1,507} & \multirow{4}{*}{3} & \multirow{4}{*}{0,681} \\
\hline & Leicht & 40,43 & & & \\
\hline & Mittel & 46,71 & & & \\
\hline & Schwer & 44,85 & & & \\
\hline \multirow{4}{*}{ Depressivität } & Keine & 37,70 & \multirow{4}{*}{5,107} & \multirow{4}{*}{3} & \multirow{4}{*}{0,164} \\
\hline & Leicht & 34,50 & & & \\
\hline & Mittel & 41,67 & & & \\
\hline & Schwer & 50,46 & & & \\
\hline \multirow{4}{*}{ Ängstlichkeit } & Keine & 37,59 & \multirow{4}{*}{4,057} & \multirow{4}{*}{3} & \multirow{4}{*}{0,255} \\
\hline & Leicht & 37,43 & & & \\
\hline & Mittel & 42,38 & & & \\
\hline & Schwer & 49,50 & & & \\
\hline \multirow{4}{*}{ Aggressivität } & Keine & 37,91 & \multirow{4}{*}{7,479} & \multirow{4}{*}{3} & \multirow{4}{*}{0,058} \\
\hline & Leicht & 27,00 & & & \\
\hline & Mittel & 44,67 & & & \\
\hline & Schwer & 50,79 & & & \\
\hline \multirow{4}{*}{ Phobische Angst } & Keine & 39,28 & \multirow{4}{*}{3,948} & \multirow{4}{*}{3} & \multirow{4}{*}{0,267} \\
\hline & Leicht & 33,57 & & & \\
\hline & Mittel & 40,00 & & & \\
\hline & Schwer & 49,17 & & & \\
\hline \multirow{4}{*}{ Paranoides Denken } & Keine & 35,87 & \multirow{4}{*}{6,082} & \multirow{4}{*}{3} & \multirow{4}{*}{0,108} \\
\hline & Leicht & 42,29 & & & \\
\hline & Mittel & 41,92 & & & \\
\hline & Schwer & 50,92 & & & \\
\hline \multirow{4}{*}{ Psychotizismus } & Keine & 37,96 & \multirow{4}{*}{4,085} & \multirow{4}{*}{3} & \multirow{4}{*}{0,252} \\
\hline & Leicht & 40,64 & & & \\
\hline & Mittel & 38,79 & & & \\
\hline & Schwer & 49,75 & & & \\
\hline
\end{tabular}

Kruskal-Wallis-Test 
Tabelle 50: Einfluss des Schweregrads der körperlichen Gewalt auf die Globalen Kennwerte des SCL 90-R

\begin{tabular}{|c|c|c|c|c|c|}
\hline $\begin{array}{l}\text { Globale Kennwerte nach } \\
\text { SCL } 90-R\end{array}$ & Schweregrad & $\begin{array}{l}\text { Mittlerer } \\
\text { Rang }\end{array}$ & $\chi$-Wert & df & p-Wert \\
\hline \multirow{4}{*}{ GSI } & Keine & 37,47 & \multirow{4}{*}{4,438} & \multirow{4}{*}{3} & \multirow{4}{*}{0,218} \\
\hline & Leicht & 37,64 & & & \\
\hline & Mittel & 41,54 & & & \\
\hline & Schwer & 50,00 & & & \\
\hline \multirow{4}{*}{ PSDI } & Keine & 38,16 & \multirow{4}{*}{3,547} & \multirow{4}{*}{3} & \multirow{4}{*}{0,315} \\
\hline & Leicht & 35,79 & & & \\
\hline & Mittel & 42,96 & & & \\
\hline & Schwer & 48,85 & & & \\
\hline \multirow{4}{*}{ PST } & Keine & 37,28 & \multirow{4}{*}{5,383} & \multirow{4}{*}{3} & \multirow{4}{*}{0,146} \\
\hline & Leicht & 38,57 & & & \\
\hline & Mittel & 39,42 & & & \\
\hline & Schwer & 51,02 & & & \\
\hline
\end{tabular}

Kruskal-Wallis-Test

\subsubsection{Schweregrad Vernachlässigung}

Wegen sehr geringer Fallzahlen wurden hier die Kategorien leicht $(1,2 \%)$ und mittelschwer (6,0\%) zusammengefasst. Unter schwerer Vernachlässigung litten $57,8 \%$ der Befragten in ihrer Kindheit, 34,9\% gaben keine derartige Beeinträchtigung an. $63 \%$ der schwer Vernachlässigten waren männlich, in den beiden anderen Kategorien bestand ein ausgewogenes Geschlechtsverhältnis. Dies entspricht einem Anteil von rund 55\% aller Männer. Die Frauen wiesen in knapp zwei Drittel der Fälle schwere Vernachlässigung in der Kindheit auf. Männer und Frauen unterscheiden sich damit nicht signifikant ( $\chi$-Wert 3,551, Freiheitsgrad 3, P-Wert 0,314, Chi-Quadrat nach Pearson, 2-seitig).

Wie auch schon in den vorangegangenen Betrachtungen stellen die folgenden Tabellen dar, ob sich Unterschiede zwischen den Schweregradkategorien hinsichtlich der Composite Scores, der Skalenwerte und der globalen Kennwerte 
ergaben. Hier fanden sich zwischen den Schweregradkategorien der Vernachlässigung keine signifikanten Unterschiede.

Tabelle 51: Einfluss des Schweregrads der Vernachlässigung auf die Composite Scores des EuropASI

\begin{tabular}{|c|c|c|c|}
\hline $\begin{array}{l}\text { Composite Scores nach } \\
\text { EuropASI }\end{array}$ & Schweregrad & $\begin{array}{l}\text { Mittlerer } \\
\text { Rang }\end{array}$ & p-Wert \\
\hline \multirow{2}{*}{ Körperliche Probleme } & Leicht \& Mittel & 29,08 & \multirow{2}{*}{0,769} \\
\hline & Schwer & 27,30 & \\
\hline \multirow{2}{*}{ Arbeit: Ökon. Situation } & Leicht \& Mittel & 27,67 & \multirow{2}{*}{0,968} \\
\hline & Schwer & 27,48 & \\
\hline \multirow{2}{*}{ Arbeit: Zufriedenheit } & Leicht \& Mittel & 23,92 & \multirow{2}{*}{0,538} \\
\hline & Schwer & 27,95 & \\
\hline \multirow{2}{*}{ Alkoholgebrauch } & Leicht \& Mittel & 25,00 & \multirow{2}{*}{0,628} \\
\hline & Schwer & 27,81 & \\
\hline \multirow{2}{*}{ Drogengebrauch } & Leicht \& Mittel & 34,00 & \multirow{2}{*}{0,283} \\
\hline & Schwer & 26,69 & \\
\hline \multirow{2}{*}{ Rechtliche Probleme } & Leicht \& Mittel & 27,33 & \multirow{2}{*}{0,978} \\
\hline & Schwer & 27,52 & \\
\hline \multirow{2}{*}{ Familie } & Leicht \& Mittel & 26,58 & \multirow{2}{*}{0,876} \\
\hline & Schwer & 27,61 & \\
\hline \multirow{2}{*}{ Sozialbeziehungen } & Leicht \& Mittel & 30,92 & \multirow{2}{*}{0,549} \\
\hline & Schwer & 27,07 & \\
\hline \multirow{2}{*}{ Psychische Probleme } & Leicht \& Mittel & 21,92 & \multirow{2}{*}{0,356} \\
\hline & Schwer & 28,20 & \\
\hline
\end{tabular}

Mann-Whitney-Test, 2-seitig

Tabelle 52: Einfluss des Schweregrads der Vernachlässigung auf die Skalenwerte des SCL 90-R

\begin{tabular}{llll}
\hline $\begin{array}{l}\text { Skalenwerte nach } \\
\text { SCL 90-R }\end{array}$ & Schweregrad & $\begin{array}{l}\text { Mittlerer } \\
\text { Rang }\end{array}$ & p-Wert \\
\hline \multirow{2}{*}{ Somatisierung } & Leicht \& Mittel & 28,00 & \multirow{2}{*}{0,934} \\
\cline { 2 - 3 } & Schwer & 27,44 & \\
\hline Zwanghaftigkeit & Leicht \& Mittel & 28,67 & 0,847 \\
\hline
\end{tabular}




\begin{tabular}{|c|c|c|c|}
\hline & Schwer & 27,35 & \\
\hline \multirow{2}{*}{ Unsicherheit Sozialkontakt } & Leicht \& Mittel & 25,83 & \multirow{2}{*}{0,783} \\
\hline & Schwer & 27,71 & \\
\hline \multirow{2}{*}{ Depressivität } & Leicht \& Mittel & 25,58 & \multirow{2}{*}{0,751} \\
\hline & Schwer & 27,74 & \\
\hline \multirow{2}{*}{ Ängstlichkeit } & Leicht \& Mittel & 31,83 & \multirow{2}{*}{0,474} \\
\hline & Schwer & 26,96 & \\
\hline \multirow{2}{*}{ Aggressivität } & Leicht \& Mittel & 29,08 & \multirow{2}{*}{0,793} \\
\hline & Schwer & 27,30 & \\
\hline \multirow{2}{*}{ Phobische Angst } & Leicht \& Mittel & 24,58 & \multirow{2}{*}{0,624} \\
\hline & Schwer & 27,86 & \\
\hline \multirow{2}{*}{ Paranoides Denken } & Leicht \& Mittel & 22,25 & \multirow{2}{*}{0,384} \\
\hline & Schwer & 28,16 & \\
\hline \multirow{2}{*}{ Psychotizismus } & Leicht \& Mittel & 31,17 & \multirow{2}{*}{0,543} \\
\hline & Schwer & 27,04 & \\
\hline
\end{tabular}

Mann-Whitney-Test, 2-seitig

Tabelle 53: Einfluss der Schweregrade der Vernachlässigung auf die Globalen Kennwerte des SCL 90-R

\begin{tabular}{llll}
\hline $\begin{array}{lll}\text { Globale Kennwerte nach } \\
\text { SCL 90-R }\end{array}$ & Schweregrad & $\begin{array}{l}\text { Mittlerer } \\
\text { Rang }\end{array}$ & p-Wert \\
\hline \multirow{2}{*}{ GSI } & Leicht \& Mittel & 30,58 & \multirow{2}{*}{0,611} \\
\cline { 2 - 3 } & Schwer & 27,11 & \\
PSDI & Leicht \& Mittel & 29,33 & \\
\cline { 2 - 3 } & Schwer & 27,27 & \multirow{2}{*}{0,989} \\
\multirow{2}{*}{ PST } & Leicht \& Mittel & 27,58 & \\
\cline { 2 - 3 } & Schwer & 27,49 & \\
\hline
\end{tabular}

Mann-Whitney-Test, 2-seitig

\subsubsection{Schweregrad familiäre Gewalt}

Knapp die Hälfte der Befragten hatte keine Erfahrungen mit familiärer Gewalt in der Kindheit gemacht. Der Rest verteilte sich nahezu gleichmäßig auf die drei Schweregradkategorien. 53\% der Männer und 44\% der Frauen hatten keine Erfahrungen mit familiärer Gewalt. Entsprechende schwere Gewalterfahrungen 
konnten 13\% der Männer und 22\% der Frauen zugeordnet werden. Männer und Frauen unterschieden sich damit nicht signifikant $(\chi$-Wert 1,725, Freiheitsgrad 3 , P-Wert 0,631, Chi-Quadrat nach Pearson, 2-seitig).

Tabelle 54 zeigt, ob sich Unterschiede in den Composite Scores zwischen den verschiedenen Schweregraden finden ließen. Bis auf die psychischen Probleme waren keine Unterschiede bezüglich des Schweregrads bei familiärer Gewalt beim EuropASI messbar. Hier setzte sich der höchste Schweregrad des Missbrauchs von dem mittleren und leichten ab, welche in etwa eine gleiche Belastung hervorriefen.

Tabelle 54: Einfluss des Schweregrads der familiären Gewalt auf die Composite Scores des EuropASI

\begin{tabular}{|c|c|c|c|c|c|}
\hline $\begin{array}{lll}\text { Composite } & \text { Scores } & \text { nach } \\
\text { EuropASI } & & \\
\end{array}$ & Schweregrad & $\begin{array}{l}\text { Mittlerer } \\
\text { Rang }\end{array}$ & $\chi$-Wert & df & p-Wert \\
\hline \multirow{4}{*}{ Körperliche Probleme } & Keine & 37,99 & \multirow{4}{*}{3,764} & \multirow{4}{*}{3} & \multirow{4}{*}{0,288} \\
\hline & Leicht & 40,85 & & & \\
\hline & Mittel & 44,57 & & & \\
\hline & Schwer & 49,69 & & & \\
\hline \multirow{4}{*}{ Arbeit: Ökon. Situation } & Keine & 38,98 & \multirow{4}{*}{5,093} & \multirow{4}{*}{3} & \multirow{4}{*}{0,165} \\
\hline & Leicht & 36,54 & & & \\
\hline & Mittel & 45,30 & & & \\
\hline & Schwer & 50,04 & & & \\
\hline \multirow{4}{*}{ Arbeit: Zufriedenheit } & Keine & 38,60 & \multirow{4}{*}{3,442} & \multirow{4}{*}{3} & \multirow{4}{*}{0,328} \\
\hline & Leicht & 37,35 & & & \\
\hline & Mittel & 48,27 & & & \\
\hline & Schwer & 47,00 & & & \\
\hline \multirow{4}{*}{ Alkoholgebrauch } & Keine & 40,76 & \multirow{4}{*}{1,784} & \multirow{4}{*}{3} & \multirow{4}{*}{0,618} \\
\hline & Leicht & 42,00 & & & \\
\hline & Mittel & 46,93 & & & \\
\hline & Schwer & 37,08 & & & \\
\hline \multirow{4}{*}{ Drogengebrauch } & Keine & 40,98 & \multirow{4}{*}{5,565} & \multirow{4}{*}{3} & \multirow{4}{*}{0,135} \\
\hline & Leicht & 30,69 & & & \\
\hline & Mittel & 42,67 & & & \\
\hline & Schwer & 52,62 & & & \\
\hline
\end{tabular}




\begin{tabular}{|c|c|c|c|c|c|}
\hline \multirow{4}{*}{ Rechtliche Probleme } & Keine & 41,26 & \multirow{4}{*}{4,100} & \multirow{4}{*}{3} & \multirow{4}{*}{0,251} \\
\hline & Leicht & 33,62 & & & \\
\hline & Mittel & 39,93 & & & \\
\hline & Schwer & 51,96 & & & \\
\hline \multirow{4}{*}{ Familie } & Keine & 38,77 & \multirow{4}{*}{1,299} & \multirow{4}{*}{3} & \multirow{4}{*}{0,729} \\
\hline & Leicht & 46,04 & & & \\
\hline & Mittel & 43,80 & & & \\
\hline & Schwer & 42,92 & & & \\
\hline \multirow{4}{*}{ Sozialbeziehungen } & Keine & 38,72 & \multirow{4}{*}{2,012} & \multirow{4}{*}{3} & \multirow{4}{*}{0,570} \\
\hline & Leicht & 47,62 & & & \\
\hline & Mittel & 41,30 & & & \\
\hline & Schwer & 44,38 & & & \\
\hline \multirow{4}{*}{ Psychische Probleme } & Keine & 35,10 & \multirow{4}{*}{8,198} & \multirow{4}{*}{3} & \multirow{4}{*}{0,042} \\
\hline & Leicht & 46,35 & & & \\
\hline & Mittel & 42,47 & & & \\
\hline & Schwer & 55,73 & & & \\
\hline
\end{tabular}

Kruskal-Wallis-Test

Dagegen wurden bei der vorgegebenen Einstufung der Schweregrade zur familiären Gewalt fast alle Kennwerte des SCL 90-R signifikant. Ausnahme war hier lediglich der Skalenwert der Zwanghaftigkeit. Die Unterteilung in die Schweregradkategorien deutet eine differenzierte psychische Symptombelastung an. $\mathrm{Zu}$ beachten ist, dass der leichte und mittlere Schweregrad in etwa eine vergleichbare Belastung hervorrief, häufig sogar der leichte Schweregrad etwas höhere Belastungswerte lieferte als der mittlere Schweregrad. Die Einteilung in Schweregrade sollte dies mit der Abstufung keine, leicht \& mittel und schwer berücksichtigen. 
Tabelle 55: Einfluss des Schweregrads der familiären Gewalt auf die Skalenwerte des SCL 90-R

\begin{tabular}{|c|c|c|c|c|c|}
\hline $\begin{array}{l}\text { Skalenwerte nach } \\
\text { SCL 90-R }\end{array}$ & Schweregrad & $\begin{array}{l}\text { Mittlerer } \\
\text { Rang }\end{array}$ & $\chi$-Wert & df & p-Wert \\
\hline \multirow{4}{*}{ Somatisierung } & Keine & $\overline{34,56}$ & \multirow{4}{*}{9,244} & \multirow{4}{*}{3} & \multirow{4}{*}{0,026} \\
\hline & Leicht & 45,12 & & & \\
\hline & Mittel & 44,23 & & & \\
\hline & Schwer & 56,62 & & & \\
\hline \multirow{4}{*}{ Zwanghaftigkeit } & Keine & 36,72 & \multirow{4}{*}{6,621} & \multirow{4}{*}{3} & \multirow{4}{*}{0,085} \\
\hline & Leicht & 44,88 & & & \\
\hline & Mittel & 39,40 & & & \\
\hline & Schwer & 55,62 & & & \\
\hline \multirow{4}{*}{ Unsicherheit Sozialkontakt } & Keine & 35,27 & \multirow{4}{*}{10,701} & \multirow{4}{*}{3} & \multirow{4}{*}{0,013} \\
\hline & Leicht & 44,23 & & & \\
\hline & Mittel & 40,37 & & & \\
\hline & Schwer & 59,73 & & & \\
\hline \multirow{4}{*}{ Depressivität } & Keine & 33,98 & \multirow{4}{*}{13,948} & \multirow{4}{*}{3} & \multirow{4}{*}{0,003} \\
\hline & Leicht & 44,62 & & & \\
\hline & Mittel & 41,63 & & & \\
\hline & Schwer & 61,96 & & & \\
\hline \multirow{4}{*}{ Ängstlichkeit } & Keine & 34,45 & \multirow{4}{*}{15,734} & \multirow{4}{*}{3} & \multirow{4}{*}{0,001} \\
\hline & Leicht & 42,88 & & & \\
\hline & Mittel & 39,77 & & & \\
\hline & Schwer & 64,35 & & & \\
\hline \multirow{4}{*}{ Aggressivität } & Keine & 36,80 & \multirow{4}{*}{8,751} & \multirow{4}{*}{3} & \multirow{4}{*}{0,033} \\
\hline & Leicht & 47,58 & & & \\
\hline & Mittel & 35,80 & & & \\
\hline & Schwer & 56,81 & & & \\
\hline \multirow{4}{*}{ Phobische Angst } & Keine & 34,45 & \multirow{4}{*}{10,743} & \multirow{4}{*}{3} & \multirow{4}{*}{0,013} \\
\hline & Leicht & 47,58 & & & \\
\hline & Mittel & 41,80 & & & \\
\hline & Schwer & 57,31 & & & \\
\hline \multirow{4}{*}{ Paranoides Denken } & Keine & 34,34 & \multirow{4}{*}{15,320} & & \\
\hline & Leicht & 43,23 & & 0 & 0000 \\
\hline & Mittel & 40,27 & & 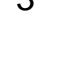 & 0,002 \\
\hline & Schwer & 63,77 & & & \\
\hline
\end{tabular}




\begin{tabular}{|c|c|c|c|c|c|}
\hline \multirow{4}{*}{ Psychotizismus } & Keine & 33,73 & \multirow{4}{*}{13,414} & \multirow{4}{*}{3} & \multirow{4}{*}{0,004} \\
\hline & Leicht & 47,92 & & & \\
\hline & Mittel & 41,17 & & & \\
\hline & Schwer & 59,96 & & & \\
\hline
\end{tabular}

Kruskal-Wallis-Test

Tabelle 56: Einfluss des Schweregrads der familiären Gewalt auf die Globalen Kennwerte des SCL $90-R$

\begin{tabular}{|c|c|c|c|c|c|}
\hline $\begin{array}{l}\text { Globale Kennwerte nach } \\
\text { SCL 90-R }\end{array}$ & Schweregrad & $\begin{array}{l}\text { Mittlerer } \\
\text { Rang }\end{array}$ & $\chi$-Wert & df & p-Wert \\
\hline \multirow{4}{*}{ GSI } & Keine & 34,10 & \multirow{4}{*}{13,932} & \multirow{4}{*}{3} & \multirow{4}{*}{0,003} \\
\hline & Leicht & 45,58 & & & \\
\hline & Mittel & 40,50 & & & \\
\hline & Schwer & 61,92 & & & \\
\hline \multirow{4}{*}{ PSDI } & Keine & 36,04 & \multirow{4}{*}{11,090} & \multirow{4}{*}{3} & \multirow{4}{*}{0,011} \\
\hline & Leicht & 45,62 & & & \\
\hline & Mittel & 36,73 & & & \\
\hline & Schwer & 60,12 & & & \\
\hline \multirow{4}{*}{ PST } & Keine & 33,49 & \multirow{4}{*}{14,067} & \multirow{4}{*}{3} & \multirow{4}{*}{0,003} \\
\hline & Leicht & 46,88 & & & \\
\hline & Mittel & 41,80 & & & \\
\hline & Schwer & 61,04 & & & \\
\hline
\end{tabular}

Kruskal-Wallis-Test

\subsubsection{Schweregrad emotionaler Missbrauch}

Bei 36\% der Befragten fand sich kein Hinweis auf emotionalen Missbrauch in der Kindheit. Jeweils 18\% zeigten einen leichten bis mittleren Schweregrad, knapp $28 \%$ waren mit dieser Missbrauchserfahrung schwer belastet. Je $32 \%$ der Frauen wiesen keine bzw. schwere Erfahrungen mit emotionalem Missbrauch auf. Bei den Männern waren 38\% der Befragten nicht betroffen, rund ein Viertel zeigte den höchsten Schweregrad an emotionalem Missbrauch. Männer und Frauen unterschieden sich damit nicht signifikant $(\chi$-Wert 2,875, Freiheitsgrad 3 , P-Wert 0,411, Chi-Quadrat nach Pearson, 2-seitig). 
Hier ergaben sich signifikante Ergebnisse in den Kennwerten des EuropASI für körperliche Probleme ( $p=0,004)$, Arbeit und Unterhalt: Ökonomische Situation $(p=0,011)$ und psychische Probleme $(p=0,021) . Z u$ beachten ist, dass Patienten ohne Missbrauch teilweise höhere Belastungswerte zeigten als bei einem leichten Missbrauch. Auch die Belastungswerte für mittleren und hohen Schweregrad des Missbrauchs lagen sehr ähnlich. Eine Einteilung der Schweregrade in zwei Gruppen mit keinem \& leichten Missbrauch sowie mittleren und schweren Missbrauch bietet sich an.

Tabelle 57: Einfluss des Schweregrads des emotionalen Missbrauchs auf die Composite Scores des EuropASI

\begin{tabular}{|c|c|c|c|c|c|}
\hline $\begin{array}{lll}\text { Composite } & \text { Scores } & \text { nach } \\
\text { EuropASI } & & \end{array}$ & Schweregrad & $\begin{array}{l}\text { Mittlerer } \\
\text { Rang }\end{array}$ & $\chi$-Wert & df & p-Wert \\
\hline \multirow{4}{*}{ Körperliche Probleme } & Keine & 37,02 & \multirow{4}{*}{13,343} & \multirow{4}{*}{3} & \multirow{4}{*}{0,004} \\
\hline & Leicht & 30,97 & & & \\
\hline & Mittel & 46,73 & & & \\
\hline & Schwer & 52,61 & & & \\
\hline \multirow{4}{*}{ Arbeit: Ökon. Situation } & Keine & 37,82 & \multirow{4}{*}{11,200} & \multirow{4}{*}{3} & \multirow{4}{*}{0,011} \\
\hline & Leicht & 33,13 & & & \\
\hline & Mittel & 53,00 & & & \\
\hline & Schwer & 46,07 & & & \\
\hline \multirow{4}{*}{ Arbeit: Zufriedenheit } & Keine & 38,12 & \multirow{4}{*}{1,493} & \multirow{4}{*}{3} & \multirow{4}{*}{0,684} \\
\hline & Leicht & 45,33 & & & \\
\hline & Mittel & 43,43 & & & \\
\hline & Schwer & 43,96 & & & \\
\hline \multirow{4}{*}{ Alkoholgebrauch } & Keine & 42,80 & \multirow{4}{*}{2,162} & \multirow{4}{*}{3} & \multirow{4}{*}{0,540} \\
\hline & Leicht & 43,23 & & & \\
\hline & Mittel & 46,57 & & & \\
\hline & Schwer & 37,17 & & & \\
\hline \multirow{4}{*}{ Drogengebrauch } & Keine & 43,23 & \multirow{4}{*}{0,509} & \multirow{4}{*}{3} & \multirow{4}{*}{0,917} \\
\hline & Leicht & 44,00 & & & \\
\hline & Mittel & 38,53 & & & \\
\hline & Schwer & 41,35 & & & \\
\hline
\end{tabular}




\begin{tabular}{|c|c|c|c|c|c|}
\hline \multirow{4}{*}{ Rechtliche Probleme } & Keine & 45,93 & \multirow{4}{*}{3,237} & \multirow{4}{*}{3} & \multirow{4}{*}{0,357} \\
\hline & Leicht & 32,60 & & & \\
\hline & Mittel & 41,43 & & & \\
\hline & Schwer & 43,37 & & & \\
\hline \multirow{4}{*}{ Familie } & Keine & 36,15 & \multirow{4}{*}{3,188} & \multirow{4}{*}{3} & \multirow{4}{*}{0,364} \\
\hline & Leicht & 43,20 & & & \\
\hline & Mittel & 46,83 & & & \\
\hline & Schwer & 45,70 & & & \\
\hline \multirow{4}{*}{ Sozialbeziehungen } & Keine & 35,47 & \multirow{4}{*}{5,165} & \multirow{4}{*}{3} & \multirow{4}{*}{0,160} \\
\hline & Leicht & 44,27 & & & \\
\hline & Mittel & 50,13 & & & \\
\hline & Schwer & 43,74 & & & \\
\hline \multirow{4}{*}{ Psychische Probleme } & Keine & 33,58 & \multirow{4}{*}{9,715} & \multirow{4}{*}{3} & \multirow{4}{*}{0,021} \\
\hline & Leicht & 41,97 & & & \\
\hline & Mittel & 40,10 & & & \\
\hline & Schwer & 54,24 & & & \\
\hline
\end{tabular}

Kruskal-Wallis-Test

Signifikanz konnte für die Skalenwerte Ängstlichkeit $(p=0,015)$, paranoides Denken $(p=0,032)$ und Psychotizismus $(p=0,029)$ des SCL 90-R nachgewiesen werden, die globalen Kennwerte zeigten keine Unterschiede bezüglich des Schweregrads des emotionalen Missbrauchs auf. Die Werte legen nahe, dass eine Abstufung des Schweregrads in kein, leicht \& mittel und schwer sinnvoll ist.

Tabelle 58: Einfluss des Schweregrads des emotionalen Missbrauchs auf die Skalenwerte des SCL 90-R

\begin{tabular}{|c|c|c|c|c|c|}
\hline $\begin{array}{l}\text { Skalenwerte nach } \\
\text { SCL } 90-R\end{array}$ & Schweregrad & $\begin{array}{l}\text { Mittlerer } \\
\text { Rang }\end{array}$ & $x$-Wert & df & p-Wert \\
\hline \multirow{4}{*}{ Somatisierung } & Keine & 36,05 & \multirow{4}{*}{7,701} & \multirow{4}{*}{3} & \multirow{4}{*}{0,053} \\
\hline & Leicht & 34,53 & & & \\
\hline & Mittel & 45,93 & & & \\
\hline & Schwer & 52,07 & & & \\
\hline Zwanghaftigkeit & Keine & 37,63 & 4,489 & 3 & 0,213 \\
\hline
\end{tabular}




\begin{tabular}{|c|c|c|c|c|c|}
\hline & Leicht & 39,07 & & & \\
\hline & Mittel & 39,97 & & & \\
\hline & Schwer & 50,93 & & & \\
\hline \multirow{4}{*}{ Unsicherheit Sozialkontakt } & Keine & 38,02 & \multirow{4}{*}{4,987} & \multirow{4}{*}{3} & \multirow{4}{*}{0,173} \\
\hline & Leicht & 47,87 & & & \\
\hline & Mittel & 34,17 & & & \\
\hline & Schwer & 48,48 & & & \\
\hline \multirow{4}{*}{ Depressivität } & Keine & 35,23 & \multirow{4}{*}{6,950} & \multirow{4}{*}{3} & \multirow{4}{*}{0,074} \\
\hline & Leicht & 42,23 & & & \\
\hline & Mittel & 39,20 & & & \\
\hline & Schwer & 52,50 & & & \\
\hline \multirow{4}{*}{ Ängstlichkeit } & Keine & 36,12 & \multirow{4}{*}{10,416} & \multirow{4}{*}{3} & \multirow{4}{*}{0,015} \\
\hline & Leicht & 43,23 & & & \\
\hline & Mittel & 32,97 & & & \\
\hline & Schwer & 54,76 & & & \\
\hline \multirow{4}{*}{ Aggressivität } & Keine & 38,70 & \multirow{4}{*}{4,598} & \multirow{4}{*}{3} & \multirow{4}{*}{0,204} \\
\hline & Leicht & 42,33 & & & \\
\hline & Mittel & 35,30 & & & \\
\hline & Schwer & 50,46 & & & \\
\hline \multirow{4}{*}{ Phobische Angst } & Keine & 37,22 & \multirow{4}{*}{6,533} & \multirow{4}{*}{3} & \multirow{4}{*}{0,088} \\
\hline & Leicht & 49,40 & & & \\
\hline & Mittel & 33,77 & & & \\
\hline & Schwer & 48,78 & & & \\
\hline \multirow{4}{*}{ Paranoides Denken } & Keine & 35,20 & \multirow{4}{*}{8,833} & \multirow{4}{*}{3} & \multirow{4}{*}{0,032} \\
\hline & Leicht & 46,23 & & & \\
\hline & Mittel & 34,87 & & & \\
\hline & Schwer & 52,76 & & & \\
\hline \multirow{4}{*}{ Psychotizismus } & Keine & 33,45 & \multirow{4}{*}{9,028} & \multirow{4}{*}{3} & \multirow{4}{*}{0,029} \\
\hline & Leicht & 43,90 & & & \\
\hline & Mittel & 40,20 & & & \\
\hline & Schwer & 53,09 & & & \\
\hline
\end{tabular}

Kruskal-Wallis-Test 
Tabelle 59: Einfluss des Schweregrads des emotionalen Missbrauchs auf die Globalen Kennwerte des SCL 90-R

\begin{tabular}{|c|c|c|c|c|c|}
\hline $\begin{array}{l}\text { Globale Kennwerte nach } \\
\text { SCL } 90-R\end{array}$ & Schweregrad & $\begin{array}{l}\text { Mittlerer } \\
\text { Rang }\end{array}$ & $\chi$-Wert & df & p-Wert \\
\hline \multirow{4}{*}{ GSI } & Keine & 35,53 & \multirow{4}{*}{7,780} & \multirow{4}{*}{3} & \multirow{4}{*}{0,051} \\
\hline & Leicht & 41,03 & & & \\
\hline & Mittel & 38,27 & & & \\
\hline & Schwer & 53,50 & & & \\
\hline \multirow{4}{*}{ PSDI } & Keine & 35,67 & \multirow{4}{*}{7,384} & \multirow{4}{*}{3} & \multirow{4}{*}{0,061} \\
\hline & Leicht & 42,07 & & & \\
\hline & Mittel & 37,67 & & & \\
\hline & Schwer & 53,04 & & & \\
\hline \multirow{4}{*}{ PST } & Keine & 36,22 & \multirow{4}{*}{5,253} & \multirow{4}{*}{3} & \multirow{4}{*}{0,154} \\
\hline & Leicht & 42,07 & & & \\
\hline & Mittel & 39,40 & & & \\
\hline & Schwer & 51,20 & & & \\
\hline
\end{tabular}

Kruskal-Wallis-Test

\subsubsection{Absolute Belastung der Missbrauchsgruppen}

In den vorigen Kapiteln wurde bereits fallweise auf die Unterschiede der mittleren Symptomwerte für die Missbrauchsgruppen hingewiesen. Gemessen wurden für die einzelnen Missbrauchsgruppen die jeweiligen Mittelwerte der Composite Scores mit EuropASI sowie der Skalenwerte und der globalen Kennwerte nach SCL 90-R.

\section{EuropASI}

Die Analyse dieser Mittelwerte zeigt auf den ersten Blick eine niedrige bis mittlere Belastung der Patienten. So lagen die Composite Scores für körperliche Probleme bei 0,25, für Arbeitsprobleme bezüglich der Zufriedenheit bei ca. 0,3, Alkoholgebrauch lediglich bei 0,2 , Familienprobleme bei 0,3 und Sozialbeziehungen bei 0,2. Steigende Werte waren bei rechtlichen und psychischen Problemen mit 0,35 zu finden. In der Beschreibung zum EuropASI wurden Ergebnisse für die Composite Scores bis 0,44 als Probleme geringen 
Maßes eingestuft. Hier war aber zu berücksichtigen, dass es sich um die Mittelwerte der jeweiligen Gruppe handelt. Die Mehrzahl der Patienten wiesen für den einzelnen Problembereich eine niedrige Ausprägung des gemessenen Wertes auf, einzelne Patienten aber durchaus hohe Werte. Im Mittel nivellierten sich diese vereinzelten hohen Ausprägungen jedoch. Eine individuelle Problembehandlung für Patienten mit einer hohen Ausprägung eines Problembereichs bleibt dabei weiterhin gültig. Hohe Problemwerte wurden bei der Arbeit- und Unterhaltssituation mit Ergebnissen um die 0,8 und beim Drogengebrauch mit 0,7 gemessen.

Tabelle 60: Vergleich der Mittelwerte der Composite Scores für die verschiedenen Missbrauchsgruppen

\begin{tabular}{llllllll}
\hline $\begin{array}{l}\text { Composite Scores } \\
\text { nach EuropASI (MW) }\end{array}$ & $\begin{array}{l}\text { Kein } \\
\text { Missb. }\end{array}$ & $\begin{array}{l}\text { Allg. } \\
\text { Missb. }\end{array}$ & $\begin{array}{l}\text { Sex. } \\
\text { Missb. }\end{array}$ & $\begin{array}{l}\text { Körp. } \\
\text { Missb. }\end{array}$ & $\begin{array}{l}\text { Ver- } \\
\text { nachl. }\end{array}$ & $\begin{array}{l}\text { Fam. } \\
\text { Gew. }\end{array}$ & $\begin{array}{l}\text { Emot. } \\
\text { Missb. }\end{array}$ \\
\hline Körperliche Probleme & 0,02 & 0,23 & $\mathbf{0 , 3 2}$ & 0,28 & 0,25 & 0,25 & 0,24 \\
\hline Arbeit: Ökon. Situation & 0,74 & 0,79 & 0,74 & 0,82 & 0,85 & $\mathbf{0 , 8 6}$ & 0,84 \\
\hline Arbeit: Zufriedenheit & 0,33 & 0,27 & 0,31 & 0,29 & 0,31 & $\mathbf{0 , 3 2}$ & 0,30 \\
\hline Alkoholgebrauch & 0,24 & 0,18 & 0,14 & 0,21 & $\mathbf{0 , 1 9}$ & $\mathbf{0 , 1 9}$ & 0,16 \\
\hline Drogengebrauch & 0,69 & 0,68 & 0,68 & $\mathbf{0 , 7 1}$ & $\mathbf{0 , 7 1}$ & 0,70 & 0,68 \\
\hline Rechtliche Probleme & 0,35 & 0,35 & 0,35 & $\mathbf{0 , 3 8}$ & 0,37 & 0,36 & 0,33 \\
\hline Familie & 0,23 & 0,29 & 0,29 & $\mathbf{0 , 3 4}$ & 0,32 & 0,32 & 0,32 \\
\hline Sozialbeziehungen & 0,04 & 0,18 & 0,20 & $\mathbf{0 , 2 3}$ & 0,20 & 0,19 & 0,19 \\
\hline Psychische Probleme & 0,34 & 0,34 & $\mathbf{0 , 4 3}$ & 0,37 & 0,37 & 0,37 & 0,34 \\
\hline Höchster Wert eines Composite Scores jeweils im Fettdruck herausgehoben & & & &
\end{tabular}

Unter Beachtung der obigen Ausführungen lässt sich daraus die Folgerung ableiten, dass bezüglich dieser beiden Problembereiche die Mehrheit der Patienten eine starke Belastung aufwiesen. Dabei waren die Ergebnisse des Problembereichs Drogengebrauch auf Grund der ausgewählten Stichprobe der Patienten offensichtlich. Die Arbeits- und Unterhaltssituation war bei Drogenpatienten ebenfalls sehr kritisch, wie die Auswertungen hier auch wiedergaben. 
Als nächstes sollen die Ergebnisse der Missbrauchsgruppen untereinander verglichen werden. Bei den in Tabelle 60 eingetragen Mittelwerten der Composite Scores ist durch Fettdruck der jeweils höchste Wert für einen Problembereich hervorgehoben und der Missbrauchsgruppe zugeordnet. Es zeigte sich hierbei eine Streuung der Maximalwerte über die Gruppen sexueller Missbrauch, körperlicher Misshandlung, Vernachlässigung und familiäre Gewalt.

\section{SCL 90-R}

Ebenso wie die Ergebnisse des EuropASI sollen auch die Kennwerte des SCL 90-R bezüglich der Missbrauchsgruppen analysiert werden. In Kürze genannt seien die Größenordnungen der Skalenwerte für Phobische Angst $(0,6)$, Psychotizismus $(0,7)$, Unsicherheit im Sozialkontakt $(1,0)$, Somatisierung $(1,1)$, Paranoides Denken (1,1), Aggressivität $(1,1)$, Ängstlichkeit $(1,2)$, Zwanghaftigkeit $(1,3)$ und Depressivität $(1,5)$. Aus Tabelle 61 ist ersichtlich, dass die Skala Depressivität die höchsten durchschnittlichen Belastungswerte mit sich brachte. Die höchsten Mittelwerte über alle Skalen fanden sich bei der Gruppe mit sexueller Missbrauchserfahrung. Das bedeutet, dass die in der Kindheit sexuell missbrauchten Drogenpatienten die höchste psychische Symptombelastung zu bewältigen hatten. Dieses Ergebnis deutet auf die Auswirkungen der Traumatisierung durch sexuellen Missbrauch in der Kindheit auf die spätere psychische Symptombelastung der Patienten hin - insbesondere auch im direkten Vergleich mit anderen Traumatisierungen.

Ein Vergleich der mittleren Skalenwerte der Gruppen mit Missbrauchserfahrung mit der Gruppe ohne Missbrauchserfahrung lässt erkennen, dass alle psychischen Symptombelastungen bei einer Missbrauchserfahrung in der Kindheit sehr deutlich über denen der Vergleichsgruppe ohne Missbrauchserfahrung lagen. Dies galt auch für die globalen Kennwerte. Eine Traumatisierung in der Kindheit zieht somit bei drogenkranken Patienten eine höhere psychische Symptombelastung in der heutigen Zeit nach sich. 
Tabelle 61: Vergleich der Mittelwerte der Skalenwerte für die verschiedenen Missbrauchsgruppen, die Gruppe ohne Missbrauch und die gesamte Stichprobe

\begin{tabular}{lcccccccc}
\hline $\begin{array}{l}\text { Skalenwerte nach } \\
\text { SCL 90-R (MW) }\end{array}$ & Allg. & Sex. & Körp. & Vern. & $\begin{array}{l}\text { Fam. } \\
\text { Gew. }\end{array}$ & Emo. & Kein & Ges. \\
\hline Somatisierung & 1,05 & $\mathbf{1 , 2 0}$ & 1,09 & 1,14 & 1,18 & 1,11 & 0,59 & 0,97 \\
& & & & & & & & \\
\hline Zwanghaftigkeit & 1,21 & $\mathbf{1 , 4 4}$ & 1,22 & 1,31 & 1,31 & 1,22 & 0,88 & 1,15 \\
\hline Unsicherheit & 0,95 & $\mathbf{1 , 2 9}$ & 0,99 & 0,98 & 1,09 & 0,98 & 0,55 & 0,87 \\
Sozialkontakt & & & & & & & & \\
\hline Depressivität & 1,36 & $\mathbf{1 , 7 4}$ & 1,39 & 1,44 & 1,52 & 1,40 & 0,82 & 1,26 \\
\hline Ängstlichkeit & 1,19 & $\mathbf{1 , 3 6}$ & 1,26 & 1,31 & 1,22 & 1,22 & 0,55 & 1,07 \\
\hline Aggressivität & 1,09 & $\mathbf{1 , 2 0}$ & 1,11 & 1,15 & 1,13 & 1,10 & 0,80 & 1,03 \\
\hline Phobische Angst & 0,53 & $\mathbf{0 , 7 2}$ & 0,55 & 0,60 & 0,65 & 0,56 & 0,16 & 0,46 \\
\hline Paranoides & 1,03 & $\mathbf{1 , 3 1}$ & 1,14 & 1,08 & 1,19 & 1,09 & 0,56 & 0,95 \\
Denken & & & & & & & & \\
\hline Psychotizismus & 0,62 & $\mathbf{0 , 8 9}$ & 0,65 & 0,68 & 0,72 & 0,69 & 0,19 & 0,54 \\
\hline Höchster Wert eines Saklenwertes jeweils im Fettdruck herausgehoben & & & & \\
\hline
\end{tabular}

Die Globalen Kennwerte stellen eine übergeordnete Auswertung der einzelnen Skalenwerte zur Verfügung. Wie für die Skalen sollen auch für die globalen Kennwerte die Größenordnung der Werte zusammengefasst werden. Die Ergebnisse der Kennwerte über alle Gruppen lagen bei GSI $(1,1)$, PSDI $(2,1)$ und PST (45). Auch bei den globalen Kennwerten wies die Gruppe der sexuell missbrauchten Patienten die höchsten Werte auf (siehe Tabelle 62).

Tabelle 62: Vergleich der Mittelwerte der drei globalen Kennwerte für die verschiedenen Missbrauchsgruppen, die Gruppe ohne Missbrauch und die gesamte Stichprobe

\begin{tabular}{lllllllll}
\hline $\begin{array}{l}\text { Globale } \\
\begin{array}{l}\text { Kennwerte nach } \\
\text { SCL 90-R (MW) }\end{array}\end{array}$ & Allg. & Sex. & Körp. & Vern. & Fam. & Emo. & Kein & Ges. \\
Gew. & & & & & & & & \\
\hline GSI & 1,04 & $\mathbf{1 , 2 9}$ & 1,08 & 1,12 & 1,16 & 1,08 & 0,59 & 0,96 \\
\hline PSDI & 2,01 & $\mathbf{2 , 1 0}$ & 2,04 & 2,05 & 2,05 & 2,04 & 1,63 & 1,95 \\
\hline PST & 43,13 & $\mathbf{5 1 , 0 7}$ & 44,02 & 45,98 & 47,28 & 43,89 & 28,86 & 40,55 \\
\hline Höchster Wert eines Globalen Kennwertes jeweils im Fettdruck herausgehoben &
\end{tabular}




\section{Diskussion}

Die Ergebnisse haben gezeigt, dass die untersuchte Studienpopulation einer Vielzahl von belastenden Faktoren in der Kindheit ausgesetzt war. Mittels SCL 90-R konnte für jede der Missbrauchsgruppen die Art und Schwere der psychischen Symptombelastung, mittels EuropASI die Art und Schwere der Belastung in den Lebensbereichen gemessen werden. Hier war zu erkennen, dass vor allem bei den psychischen Erkrankungen eine signifikant höhere Symptombelastung bei den Patientengruppen mit einer Missbrauchsvorgeschichte zu finden war. Außerdem teilten wir die Ergebnisse nach Geschlechtern auf, um festzustellen, ob sich Unterschiede zwischen Männern und Frauen finden lassen. In einigen wenigen Punkten wie Unsicherheit im Sozialkontakt zu anderen Menschen und phobischen Angstzuständen ließ sich hier eine signifikant stärkere Belastung der Frauen nachweisen. Ebenso untersuchten wir den Einfluss des Schweregrads des jeweiligen Missbrauchs. Hier war vor allem bei sexuellem Missbrauch und familiärer Gewalt eine wesentlicher Unterschied in der psychischen Symptombelastung vorzufinden.

Im folgenden sollen nun unsere Ergebnisse der Literatur gegenübergestellt und diskutiert werden.

\subsection{Diskussion des Einflusses von Missbrauch auf den Krankheitsverlauf}

\subsubsection{Einfluss von Missbrauch auf die Entwicklung spezifischer Symptome und die Suchtschwere}

Eine Kernfrage der Studie lautete, ob die Gruppe der in der Kindheit missbrauchten drogenabhängigen Patienten spezifische Symptome und Probleme im Erwachsenenalter aufweist, die in der Referenzgruppe der drogenabhängigen Patienten ohne Missbrauchserfahrung nicht zu finden sind. In 
der Literatur finden sich dazu Hinweise auf Symptomkonstellationen, die gehäuft bei körperlichem und sexuellem Missbrauch beschrieben wurden. Hierzu zählen psychische Probleme wie erhöhte Autoaggression und Suizidneigung, Weglauftendenzen in der Kindheit, dissoziale Tendenzen, erhöhte Gewaltbereitschaft, ebenso soziale Probleme wie soziale Isolation, aber auch Prostitution und Reviktimisierungen (siehe auch bei Hirsch 1987, Kendall-Tackett et al. 1993, Moggi 1997).

\subsubsection{1 Übersicht des Einflusses von Missbrauch auf die Problembereiche nach EuropASI}

In unserer Untersuchung hatten wir die in Kapitel 3.2 beschriebene, standardisierte Messmethode des EuropASI verwendet, welcher wichtige Indikatoren über die Lebensbereiche der Patienten erfasst. Ziel war eine Einschätzung des Zustandes der Patienten bezüglich dieser verschiedenen Lebensbereiche - beschrieben durch die Kennwerte der Composite Scores - zu gewinnen. In Tabelle 63 wurden jene Felder dunkel eingefärbt, bei denen sich die Composite Scores signifikant zwischen Patienten mit und ohne Missbrauchserfahrung unterschieden, wobei jeweils die Missbrauchsgruppe signifikant schlechtere Werte aufwies. Die Übersicht zeigt, dass die Missbrauchsgruppe signifikant mehr Probleme bei „Psychischen Probleme“ und „Körperlichen Probleme“ sowie abgeschwächt bei „Sozialbeziehungen“ und „Arbeit/ökonomische Situation“ hatte.

Tabelle 63: Signifikante Composite Scores nach EuropASI für die verschiedenen Missbrauchsgruppen

\begin{tabular}{|l|l|l|l|l|l|l|}
\hline $\begin{array}{l}\text { Signfikante Composite } \\
\text { Scores nach Europ ASI }\end{array}$ & $\begin{array}{l}\text { Allg. } \\
\text { Missb }\end{array}$ & $\begin{array}{l}\text { Sex. } \\
\text { Missb. }\end{array}$ & $\begin{array}{l}\text { Körp. } \\
\text { Missb. }\end{array}$ & $\begin{array}{l}\text { Vernach- } \\
\text { lässigung }\end{array}$ & $\begin{array}{l}\text { Fam. } \\
\text { Gewalt }\end{array}$ & $\begin{array}{l}\text { Emot. } \\
\text { Missb. }\end{array}$ \\
\hline Körperliche Probleme & & & & & & \\
\hline Arbeit: Ökon. Situation & & & & & & \\
\hline Arbeit: Zufriedenheit & & & & & & \\
\hline Alkoholgebrauch & & & & & & \\
\hline
\end{tabular}




\begin{tabular}{|l|l|l|l|l|l|l|}
\hline Drogengebrauch & & & & & & \\
\hline Rechtliche Probleme & & & & & & \\
\hline Familie & & & & & & \\
\hline Sozialbeziehungen & & & & & & \\
\hline Psychische Probleme & & & & & & \\
\hline
\end{tabular}

Hellere Schraffierung kennzeichnet signifikante Werte mit $\mathrm{p}<0,05$, dunklere Schraffierung hochsignifikante Werte mit $\mathrm{p}<$ 0,001

Im Folgenden wird auf die auffälligsten Probleme und Symptome im Zusammenhang mit Missbrauch sowie die Fragestellung „Einfluss des Missbrauchs auf die Suchtschwere" eingegangen.

\subsubsection{Einfluss von Missbrauch auf die Entwicklung körperlicher Probleme} Aus Tabelle 63 ist ersichtlich, dass bei unseren Patienten mit Missbrauchserfahrung in der Kindheit körperliche Probleme signifikant stärker ausgeprägt waren. Körperliche Probleme umfassen u.a. chronische gesundheitliche Probleme, Anzahl der Krankenhausaufenthalte, etc. Dieses Ergebnis deckt sich mit den Erkenntnissen der Hamburger Studie zu sexuellen Traumatisierungen und körperlichen Misshandlungen durch Richter-Appelt et al. (1995). Dabei wurde an einer nicht-klinischen Stichprobe von 462 Studenten und 623 Studentinnen untersucht, inwiefern Adoleszente, die in ihrer Kindheit körperliche und/oder sexuelle Misshandlung erfahren haben, sich untereinander, aber vor allem von nicht durch Missbrauch und Misshandlungen betroffenen Kontrollpersonen unterscheiden. Insgesamt gaben 23\% der Frauen und 4\% der Männer an, sexuell und 28\% der Frauen und $14 \%$ der Männer, körperlich missbraucht worden zu sein. Die Frauen berichteten über körperliche Beschwerden, aber auch über psychische Probleme wie Autoaggressionen und sexuelle Probleme. 


\subsubsection{Einfluss von Missbrauch auf die Entwicklung psychischer Probleme (SCL 90-R)}

Tabelle 63 mit den Ergebnissen nach EuropASI zeigte bereits, dass psychische Probleme in unseren Ergebnissen bei allen Missbrauchsgruppen signifikant schwerwiegender auftraten, als bei den Vergleichsgruppen ohne die jeweilige Missbrauchserfahrung. Eine detaillierte Untersuchung der psychischen Probleme erlaubte die Erfassung mit SCL 90-R, dessen Ergebnisse in Tabelle 64 zusammengestellt sind. Beim SCL 90-R wurden die einzelnen psychischen Symptome den Missbrauchsarten gegenübergestellt. Analog zu der obigen Vorgehensweise wurden auch in dieser Tabelle wieder die Werte, die zwischen den Gruppen mit und ohne Missbrauch signifikant geworden sind, grau eingefärbt, die hochsignifikanten Werte dunkelgrau.

Tabelle 64: Signifikante Kennwerte nach SCL 90-R für die verschiedenen Missbrauchsgruppen

\begin{tabular}{|l|l|l|l|l|l|l|}
\hline $\begin{array}{l}\text { Signfikante Kennwerte nach } \\
\text { SCL 90-R }\end{array}$ & $\begin{array}{l}\text { Allg. } \\
\text { Missb }\end{array}$ & $\begin{array}{l}\text { Sex. } \\
\text { Missb. }\end{array}$ & $\begin{array}{l}\text { Körp. } \\
\text { Missb. }\end{array}$ & $\begin{array}{l}\text { Vernach- } \\
\text { lässigung }\end{array}$ & $\begin{array}{l}\text { Fam. } \\
\text { Gewalt }\end{array}$ & $\begin{array}{l}\text { Emot. } \\
\text { Missb. }\end{array}$ \\
\hline Somatisierung & & & & & & \\
\hline Zwanghaftigkeit & & & & & & \\
\hline Unsicherheit Sozialkontakt & & & & & & \\
\hline Depressivität & & & & & & \\
\hline Ängstlichkeit & & & & & & \\
\hline Aggressivität & & & & & & \\
\hline Phobische Angst & & & & & & \\
\hline Paranoides Denken & & & & & & \\
\hline Psychotizismus & & & & & & \\
\hline GSI & & & & & & \\
\hline PSDI & & & & & & \\
\hline PST & & & & & \\
\hline $\begin{array}{l}\text { Hellere Schraffierung kennzeichnet signifikante Werte mit } p<0,05, \text { dunklere Schraffierung hochsignifikante Werte } \\
\text { mit } p<0,001\end{array}$ & &
\end{tabular}

Bemerkenswert war die breit angelegte psychische Symptombelastung: Die überwiegende Mehrzahl der psychischen Symptome wies eine signifikante 
Mehrbelastung für die Gruppe mit einer jeweiligen Missbrauchserfahrung gegenüber der Vergleichsgruppe ohne diesen Missbrauch auf. Ähnliches findet sich auch in der Literatur: In der Studie von Rounsaville et al. (1982) stellten sich für die Gruppe mit zusätzlich feststellbarem Kindheitstrauma gegenüber primär Süchtigen und primär straffälligen Süchtigen die schlechtesten therapeutischen Chancen heraus. Diese Untergruppe war durch schwere Defizite in der IchFunktionen (ego-functioning) charakterisiert. Sie waren für impulsive, unkontrollierte Handlungen und Affekte anfällig und schienen in ihrer Selbstkontrolle durch dysphorisch-gereizte Stimmungslagen, Schuldgefühle und Ängste behindert. Die Gruppe der traumatisierten Patienten wies zudem die höchste Rate an dissozialen Persönlichkeitszügen und die geringsten sozialen Kompetenzen auf. Analog beschrieben diesen Zusammenhang auch Hirsch 1987, Kendall-Tackett et al. 1993 und Moggi 1997.

Angemerkt werden sollte, dass familiäre Gewalt und emotionaler Missbrauch signifikante Unterschiede zwischen den Gruppen mit und ohne Missbrauch aufzeigten, diese Missbrauchsarten aber in der Literatur so gut wie nicht einzeln erfasst, sondern unter den anderen Missbrauchsarten, vor allem Vernachlässigung, subsummiert werden.

Im Einzelnen wird nun auf den Zusammenhang zwischen psychischen Problemen und sexuellem Missbrauch sowie Vernachlässigung eingegangen, die die stärkste psychische Symptombelastung zur Folge hatten (vgl. Tabelle 64).

\subsubsection{Einfluss des sexuellen Missbrauchs auf die Entwicklung psychischer Probleme}

Wie in Tabelle 64 ersichtlich waren bis auf Zwanghaftigkeit alle psychischen Symptome signifikant schlechter für die Missbrauchsgruppe. Diese starke Symptombelastung als Folge sexuellen Missbrauchs bestätigt auch die Literatur: Nach Briere (1992) führte sexueller Missbrauch zu einem negativen Selbstbild, chronischer Wahrnehmung von Gefahr und Ungerechtigkeit, Machtlosigkeit und 
Beschäftigung mit Kontrolle, dissoziativer Kontrolle über das Bewusstsein und beeinträchtigten Selbstbezügen. Bei unseren Ergebnissen waren die Patienten mit sexueller Missbrauchserfahrung teilweise hochsignifikant durch ein negatives Selbstbild, phobische Angstzustände, Entfremdung, Depressivität, etc. geprägt.

Nach den Untersuchungen von Marcenko et al. (2000) korrelierte sexueller Missbrauch signifikant mit späteren psychischen Erkrankungen. Mullen et al. (1993) fanden eine positive Korrelation zwischen einem sexuellen Missbrauch in der Kindheit und psychischen Problemen im Erwachsenenalter. Auch die Untersuchung von Richter Appelt (1995) zeigte hoch signifikante Zusammenhänge zwischen Missbrauch und Misshandlung mit psychischen Problemen und Symptomen im Jugend- und Erwachsenenalter auf.

Unsere Ergebnisse bestätigten in Analogie zur Literatur, dass Drogenkranke mit sexuellem Missbrauch in der Kindheit psychisch sehr viel stärker belastet sind. Eine erhöhte Symptombelastung auf Grund des Missbrauchs ist hier eindeutig zu belegen.

\subsubsection{Einfluss der Vernachlässigung auf die Entwicklung psychischer Probleme}

In Tabelle 63 waren bei den Patienten mit Vernachlässigung mit „körperlichen Problemen“, „Arbeit: Ökonomische Situation“ „Sozialbeziehung“ und „psychischen Problemen" vier Lebensbereiche signifikant schlechter gegenüber der Vergleichsgruppe. Vernachlässigung war damit nominell sogar die stärkste beeinflussende Missbrauchsform, was die Anzahl der signifikant gewordenen Problembereiche nach EuropASI anbelangt.

Mit der detaillierteren Untersuchung der einzelnen psychischen Probleme nach SCL 90-R in Tabelle 64 war es wiederum möglich, bei Vernachlässigung viele und teilweise hochsignifikante psychische Probleme gegenüber den Patienten ohne Vernachlässigung in der Kindheit nachzuweisen. Dieser von uns 
festgestelle starke Zusammenhang zwischen Vernachlässigung Heranwachsender und späterer psychischer Störungen wurde u.a. auch durch Swett und Halpert (1993) bestätigt.

Vernachlässigung wird in der Literatur eine große Bedeutung bei der Erklärung psychischer Probleme zugewiesen. Eine Vielzahl von Autoren (Dührssen 1984, Bryer et al. 1987, Schepank 1987, Lösel et. al. 1989, Egle 1997, Fischer 1998, etc.) haben sich dazu mit dem Zusammenwirken von Risiko- und Schutzfaktoren beschäftigt, um der Frage nach den Ursachen und Auswirkungen von späteren neurotischen oder psychosomatischen Erkrankungen nachzugehen. Nach Egle (1997) tritt beispielsweise die fehlende tragfähige Beziehung zu den Eltern als größter Risikofaktor für eine psychiatrische Erkrankung auf.

In unseren Ergebnissen konnte ein signifikanter Zusammenhang zwischen Vernachlässigung in der Kindheit und den psychischen Problemen „Somatisierung“, „Zwanghaftigkeit“, Depressivität“, „Ängstlichkeit“, „Aggressivität“, „Phobische Angst“, „Paranoides Denken“ und „Psychotizismus“ bestätigt werden.

\subsubsection{Einfluss der körperlichen Misshandlung auf die Entwicklung psychischer Probleme}

In der Literatur wird der körperlichen Misshandlung ein starker Einfluss auf die Entwicklung psychischer Probleme zugeordnet. So wiesen Swett und Halpert (1993) auf die psychischen Störungen als Folge körperlicher Missbrauchserfahrung hin, was sich in unseren Ergebnissen so nicht wiederspiegelt: Die Gruppe der Patienten mit körperlicher Missbrauchserfahrung war lediglich in der Skala „Paranoides Denken“ signifikant schlechter als die Vergleichsgruppe ohne die entsprechende Missbrauchserfahrung. Alle anderen psychischen Probleme blieben unauffällig. Damit zeigte bei uns die Gruppe der körperlich missbrauchten Patienten nur wenige Unterschiede bezüglich der psychischen Problemen zur Vergleichsgruppe ohne körperliche 
Missbrauchserfahrung.

Die Ergebnisse von Sedney und Brooks (1984) sowie Briere und Runtz (1988), die in Abhängigkeit vom Schweregrad des Missbrauchs über eine erhöhte Rate von Selbstverletzungen und Suizidhandlungen sowie Schlafstörungen, Somatisierungs- und dissoziativen Störungen berichteten, konnten bei uns für „Körperliche Probleme“ (vgl. Tabelle 63) sowie „Paranoides Denken“ (vgl. Tabelle 64) bestätigt werden.

Im Gegensatz zur Literatur zeigen unsere Ergebnisse für die Auswirkungen von körperlicher Misshandlung - gerade im direkten Vergleich mit den Auswirkungen von sexuellem Missbrauch oder Vernachlässigung - lediglich einen geringen Einfluss auf psychische Probleme.

\subsubsection{Einfluss des Missbrauchs auf die Suchtschwere}

Überraschenderweise wiesen die Gruppen mit und ohne Missbrauchserfahrung keine Unterschiede bezüglich des Schweregrades des Drogenproblems auf: Bei keiner Missbrauchsart wurde in Tabelle 63 der Kennwert „Drogengebrauch“ signifikant. In der Vorarbeit zu dieser Studie (Schaefer et al., 2000) fanden sich dagegen signifikant höhere Mengen an Drogen und ein schlechterer Verlauf der Erkrankung bei der Gruppe von missbrauchten Drogenabhängigen. Ebenso hatten bei Cavaiola und Schiff (1988) missbrauchte Jugendliche mit früherem und schwererem Alkohol- und Drogenkonsum zu kämpfen als nicht missbrauchte Jugendliche im selben Behandlungsprogramm.

Im Gegensatz zu der Studie in der Normalbevölkerung von Cavaiola und Schiff beruhte unsere Untersuchung aber auf einer klinischen Stichprobe in einer Suchtstation. Es kann somit lediglich gefolgert werden, dass keine Unterschiede innerhalb einer Gruppe von Drogensüchtigen unter Berücksichtigung einer früheren Misshandlung zu messen waren. Weiterhin lässt sich die gleiche 
Drogenbelastung zwischen den Gruppen mit und ohne Missbrauchserfahrung eventuell durch das Kollektiv von schwer Abhängigkeitskranken erklären: Bei beiden Gruppen war bereits ein sehr hoher, absoluter Belastungswert bei der Drogensituation zu verzeichnen (durchschnittlicher Composite Score von 0,7). Somit war hier nur schwer eine weitere Steigerung bzw. Differenzierung zwischen den Gruppen mit und ohne Missbrauch möglich. Eventuell wird noch eine höherer Fallzahl benötigt, um diese Frage zu klären.

\subsubsection{Diskussion der breit angelegten psychischen Symptombelastung}

In Tabelle 64 war abzulesen, dass das Vorliegen eines Missbrauchs in fast allen Fällen zu einer signifikanten psychischen Mehrbelastung der Patienten führte. Es kann hier von einer sehr breit angelegten psychischen Symptombelastung in Folge des Missbrauchs gesprochen werden. Es stellte sich daher die Frage, woher diese breite Symptombelastung kommt.

In der Literatur wird berichtet, dass meist eine beträchtliche Überschneidung von körperlicher Misshandlung, emotionalem und sexuellem Missbrauch zu finden ist, wobei Kinder, die einer Form des Missbrauchs ausgesetzt sind, mit einer signifikant höheren Wahrscheinlichkeit auch andere Formen des Missbrauchs oder der Misshandlung erleiden (Briere \& Runtz 1990, Bilfuco, Brown und Adler 1991, Mullen et al. 1996). Diese hohe Koexistenz der Missbrauchsformen wurde auch von anderen Autoren festgestellt (z.B. Draijer 1990).

Auch in unserer Studie gaben die meisten von Missbrauch in der Kindheit Betroffenen gleichzeitig vier verschiedene der erhobenen Missbrauchsformen in den unterschiedlichsten Kombinationen an (28\%). Drei Kombinationen fanden sich bei $22 \%$, fünf verschiedene Formen gleichzeitig bei $22 \%$. Zwei Formen von Missbrauch erlebten 15\% und nur eine einzige Form von Missbrauch in ihrer Kindheit erlitten 13\%. Im Umkehrschluss lässt sich daraus folgern, dass $87 \%$ der Gruppe der Missbrauchspatienten zwei und mehr Missbrauchsarten gleichzeitig 
ertragen mussten.

Tabelle 65: Kombinationen der Missbrauchserfahrungen

\begin{tabular}{lc}
\hline $\begin{array}{l}\text { Parallele } \\
\text { Missbrauchserfahrungen }\end{array}$ & $\begin{array}{c}\text { Anzahl } \\
\text { in \% }\end{array}$ \\
\hline Eine & $13 \%$ \\
\hline Zwei & $15 \%$ \\
\hline Drei & $22 \%$ \\
\hline Vier & $28 \%$ \\
\hline Fünf & $22 \%$ \\
\hline
\end{tabular}

Hierin kann unter anderem die breit angelegte psychischen Symptombelastungen vermutet werden. Die Patienten mit Missbrauch in der Kindheit litten nicht nur unter den singulären Auswirkungen einer Missbauchsform, sondern zeigten auf Grund des Vorliegens von mehreren Formen von Missbrauch eine Vielzahl an Symptomen. Diese Wirkungskette soll am Beispiel der Traumatisierung durch sexuellen Missbrauch erläutert werden.

Nach Finkelhor und Browne (1985) greifen z.B. bei sexuellem Missbrauch vier Mechanismen: Traumatische Sexualisierung, Verrat, Stigmatisierung und Machtlosigkeit.

Die Traumatische Sexualisierung beinhaltet eine Vielfalt an Prozessen, wie etwa die unangemessene Konditionierung der sexuellen Ansprechbarkeit des Kindes und die Entwicklung von falschen Meinungen und Annahmen über sexuelles Verhalten. Verrat beinhaltet das Erschüttern des Vertrauen des Kindes, das eigentlich davon ausgeht, dass Personen, auf die es vertraut, daran interessiert und dazu imstande sind, es vor Schaden zu bewahren. Hier sind deutliche Überschneidungen mit Emotionalem Missbrauch, aber auch Vernachlässigung zu finden. Stigmatisierung umfasst all jene Mechanismen, die das positive Selbstbild des Kindes untergraben: die Schande, die sich langsam einstellt, die soziale Ächtung, die das Kind erleidet und die negativen Stereotypen, die von der 
Kultur und vom unmittelbaren Umfeld erworben werden. Als letztes schließt die Machtlosigkeit sowohl PTSD-artige Mechanismen ein (intensive Angst vor dem Tod oder vor der Verletzung durch ein unkontrollierbares Ereignis), als auch die wiederholte Frustration durch die Unfähigkeit, die schädigenden Erlebnisse zu beenden oder ihnen zu entkommen oder Hilfe von anderen zu bekommen. Über den Zusammenhang zwischen sexuellem Missbrauch und PTSD berichtete z.B. Teusch (2001). Auch De Bellis (2002) bestätigte diesen Zusammenhang: Nach seinen Untersuchungen haben 42\% - 90\% der Patienten, die unter PTSD leiden, eine sexuelle Missbrauchserfahrung in der Kindheit erlitten.

Anhand dieser Beispiele wird deutlich, dass bei nominellem Vorliegen einer Missbrauchsform wie sexuellem Missbrauch das Vorliegen und die Wirkung anderer Missbrauchsform zu berücksichtigen sind. So wirken auch die Folgen einer emotionalen Misshandlung und Vernachlässigung mit, ebenso kann sexueller Missbrauch zu Folgen wie PTSD führen. Die Vielschichtigkeit der Traumatisierung ist ein Ansatz, um die breit angelegte psychische Symptombelastung zu erklären.

\subsubsection{Diskussion der Ursächlichkeit der Symptome}

In der Literatur wird intensiv der Aspekt diskutiert, in wie weit die hier beschriebenen Symptome bei der Gruppe der Drogenpatienten mit Missbrauchserfahrung als Folge der Missbrauchserfahrung und/oder der Sucht gewertet werden können.

Hirsch (1987), Kendall-Tackett et al. (1993) und Moggi (1997) untersuchten dazu sexuell Missbrauchte ohne eine vorliegende Suchtdiagnose. Die Autoren stellten abhängig von der Altersgruppe der Patienten vermehrt auftretende Symptome wie Weglaufen, Gewaltbereitschaft, Homosexualität, Autoaggression und Prostitution fest. Weglaufen war das häufigste im Zusammenhang mit sexuellem Missbrauch in der Adoleszenz beschriebene Symptom. Nach den Schätzungen 
waren $30 \%$ bis $50 \%$ der jungen Mädchen, die von zu Hause wegliefen, Opfer eines sexuellen Missbrauchs. Spätere Homosexualität wird mit Angaben bis zu $30 \%$ als häufige Folge von Inzest diskutiert (Hirsch 1987).

Beitchman et al. (1992) fanden gehäuft sexuelle Störungen und homosexuelle Tendenzen sowie Reviktimisierungen und Suizidgefährdung bei sexuell Missbrauchten. Schei (1990) fand eine signifikant erhöhte Suizidalitätsrate bei Frauen mit sexuellem Missbrauch in der Vorgeschichte.

Eine Vielzahl der Symptome konnte in der Literatur in einen Zusammenhang mit der Missbrauchserfahrung in der Kindheit gestellt werden, ohne dass eine Drogenabhängigkeit vorlag. $\mathrm{Da}$ in unserer Untersuchung der Vergleich bei Drogenpatienten mit und ohne Missbrauch angestellt worden ist, und die psychischen Symptome bei der Gruppe mit Missbrauchserfahrung deutlich signifikant aufgetreten sind, ist auch dies ein Indiz dafür, dass die Ursache der psychischen Symptome auf den Missbrauch zurückzuführen ist. Allerdings darf die Kombination mit der vorliegenden Suchterkrankung nicht außer acht gelassen werden. Im Umkehrschluss kann gefolgert werden, dass die festegestellte erhöhte Symptombelastung nicht als spezifisch für Suchterkrankte interpretiert werden darf.

\subsubsection{Diskussion des Zusammenhangs zwischen Missbrauchsart und Stärke der Symptombelastung}

In Kapitel 3.2.9 wurde das Ergebnis zur Stärke der psychischen Symptombelastung in Abhängigkeit der jeweiligen Missbrauchsgruppen beschrieben. In Tabelle 66 ist diese Gegenüberstellung der Symptombelastung mit SCL 90-R noch einmal zu finden. Es stellte sich die Frage, ob für die Missbrauchsarten ein Muster in der Symptombelastung zu erkennen ist.

\section{Einordnung der Belastungswerte nach Missbrauchsgruppen}

Zunächst zeigte ein Vergleich der Mittelwerte der Gruppen mit und ohne 
Missbrauchserfahrung, dass die psychische Belastung der Gruppe ohne Missbrauchserfahrung deutlich niedriger ausfiel. Die Gruppe ohne Missbrauch hatte in allen psychischen Symptomen die niedrigsten Belastungswerte. Die Gruppen mit Missbrauchserfahrung lagen in Abhängigkeit von der jeweiligen Missbrauchsform in der Belastung mindestens um das 1,5-fache, maximal um das vierfache höher.

Dieses Ergebnis zeigt, dass eine Missbrauchserfahrung in der Kindheit starke Auswirkungen auf den psychischen Zustand der Patienten hat (vgl. auch Kapitel 4.1.1.3). Eine Unterscheidung der drogenkranken Patienten in Gruppen mit und ohne eine jeweiligen Missbrauchserfahrung ist auf Grund dieser deutlichen Unterschiede in der Belastung aus unserer Sicht bei einer Therapie zu berücksichtigen.

Die höchste Symptombelastung zeigte die Gruppe der in der Kindheit sexuell missbrauchten Patienten (vgl. auch Kapitel 4.1.1.4). Hier waren alle Belastungswerte höher als bei den anderen Missbrauchsformen. Dies deutet aus unserer Sicht auf die besonders starken negativen Auswirkungen von sexuellem Missbrauch in der Kindheit hin. Im folgenden wird noch näher auf diesen Aspekt eingegangen.

Bei den anderen Missbrauchsformen lag die Intensität der psychischen Symptombelastung etwa gleich auf, aber deutlich höher als bei der Gruppe ohne Missbrauchserfahrung. 
Tabelle 66: Vergleich der Mittelwerte der Skalenwerte für die verschiedenen Missbrauchsgruppen, die Gruppe ohne Missbrauch und die gesamte Stichprobe

\begin{tabular}{lcccccccc}
\hline $\begin{array}{l}\text { Skalenwerte nach } \\
\text { SCL 90-R (MW) }\end{array}$ & Allg. & Sex. & Körp. & Vern. & $\begin{array}{l}\text { Fam. } \\
\text { Gew. }\end{array}$ & Emo. & Kein & Ges. \\
\hline Somatisierung & 1,05 & $\mathbf{1 , 2 0}$ & 1,09 & 1,14 & 1,18 & 1,11 & 0,59 & 0,97 \\
& & & & & & & & \\
\hline Zwanghaftigkeit & 1,21 & $\mathbf{1 , 4 4}$ & 1,22 & 1,31 & 1,31 & 1,22 & 0,88 & 1,15 \\
\hline Unsicherheit & 0,95 & $\mathbf{1 , 2 9}$ & 0,99 & 0,98 & 1,09 & 0,98 & 0,55 & 0,87 \\
Sozialkontakt & & & & & & & & \\
\hline Depressivität & 1,36 & $\mathbf{1 , 7 4}$ & 1,39 & 1,44 & 1,52 & 1,40 & 0,82 & 1,26 \\
\hline Angstlichkeit & 1,19 & $\mathbf{1 , 3 6}$ & 1,26 & 1,31 & 1,22 & 1,22 & 0,55 & 1,07 \\
\hline Aggressivität & 1,09 & $\mathbf{1 , 2 0}$ & 1,11 & 1,15 & 1,13 & 1,10 & 0,80 & 1,03 \\
\hline Phobische Angst & 0,53 & $\mathbf{0 , 7 2}$ & 0,55 & 0,60 & 0,65 & 0,56 & 0,16 & 0,46 \\
\hline Paranoides & 1,03 & $\mathbf{1 , 3 1}$ & 1,14 & 1,08 & 1,19 & 1,09 & 0,56 & 0,95 \\
Denken & & & & & & & & \\
\hline Psychotizismus & 0,62 & $\mathbf{0 , 8 9}$ & 0,65 & 0,68 & 0,72 & 0,69 & 0,19 & 0,54 \\
\hline GSI & 1,04 & $\mathbf{1 , 2 9}$ & 1,08 & 1,12 & 1,16 & 1,08 & 0,59 & 0,96 \\
\hline PSDI & 2,01 & $\mathbf{2 , 1 0}$ & 2,04 & 2,05 & 2,05 & 2,04 & 1,63 & 1,95 \\
\hline PST & 43,1 & $\mathbf{5 1 , 0}$ & 44,0 & 45,9 & 47,2 & 43,8 & 28,8 & 40,5 \\
\hline
\end{tabular}

Höchster Skalenwert ist jeweils im Fettdruck herausgehoben; die Spalte mit sexuellem Missbrauch ist grau hnterlegt

Tabelle 67: Referenzwerte nach Franke für Normal Gesunde, HIV infizierte, substanzabhängige und schizophrene Patienten

\begin{tabular}{lllll}
\hline $\begin{array}{l}\text { Skalenwerte nach } \\
\text { SCL 90-R (MW) }\end{array}$ & Norm & HIV & $\begin{array}{l}\text { Sub. } \\
\text { Abh. }\end{array}$ & Schiz. \\
\hline Somatisierung & 0,35 & 0,79 & 0,74 & 1,11 \\
\hline Zwanghaftigkeit & 0,47 & 0,85 & 1,15 & 1,45 \\
\hline $\begin{array}{l}\text { Unsicherheit } \\
\text { Sozialkontakt }\end{array}$ & 0,41 & 0,72 & 0,86 & 1,22 \\
\hline Depressivität & 0,40 & 1,00 & 1,23 & 1,38 \\
\hline Ängstlichkeit & 0,29 & 0,78 & 1,13 & 1,33 \\
\hline Aggressivität & 0,31 & 0,60 & 0,61 & 0,91 \\
\hline Phobische Angst & 0,14 & 0,46 & 0,75 & 1,08 \\
\hline Paranoides Denken & 0,35 & 0,65 & 0,69 & 1,42 \\
\hline Psychotizismus & 0,18 & 0,59 & 0,57 & 1,18 \\
\hline
\end{tabular}




\begin{tabular}{lllll}
\hline GSI & 0,33 & 0,76 & 0,91 & 1,24 \\
\hline PSDI & 1,22 & 1,58 & - & 2,11 \\
\hline PST & 23,10 & 39,48 & - & 50,78
\end{tabular}

Die Vergleichsspalte mit schizophrenen Patienten ist hier grau hinterlegt

Vergleich der Stärke der Symptombelastung für die Gruppe mit sexueller Missbrauchserfahrung mit den Referenzwerten von Franke

Um die Stärke der Symptombelastung für substanzabhängige Patienten mit Missbrauchsvorgeschichte einordnen zu können, unternahmen wir folgenden Ansatz: Wir stellten unsere Ergebnisse der Skalenwerten und globalen Kennwerte unserer drogenkranken Patienten den Referenzwerten der verschiedenen Gruppen von Patienten bei Franke (1995) gegenüber. Es ist dabei zu erwähnen, dass Franke der Urheber des SCL 90-R ist und in seinen Studien die Referenzwerte für verschiedene Gruppen festgehalten hat.

Franke hatte seine Untersuchung mit einer Normalstichprobe, HIV-infizierten Patienten, Patienten mit Persönlichkeitsstörung, mit Essstörungen, mit neurotischen Störungen, mit Abhängigkeitserkrankungen, mit psychosomatischen Störungen und schließlich mit schizophren erkrankten Patienten durchgeführt. Die schizophrenen Patienten hatten bei Franke die höchsten Werte in der psychischen Symptombelastung zu verzeichnen (siehe Tabelle 67). Vergleicht man diese Werte der schizophrenen Patienten mit denen unserer drogenkranken Patienten mit sexueller Missbrauchserfahrung, so fand sich erstaunlicherweise eine weitgehende Übereinstimmung in der Größenordnung dieser Werte.

Die Skalen Somatisierung, Zwanghaftigkeit, Unsicherheit im Sozialkontakt und Ängstlichkeit waren bis auf eine Abweichung von höchstens 0,09 identisch. Ebenso zeigte der globale Kennwert GSI nur eine Abweichung von 0,05, der PST von 0,01 und der PSDI von 0,3. Abweichungen mit höheren Werten bei unserer Stichprobe gibt es bei Depressivität und Aggressivität. Abweichungen mit niedrigeren Werten bei unserer Stichprobe lagen lediglich bei phobischer Angst, 
paranoidem Denken und Psychotizismus vor. Insbesondere die globalen Kennwerte, die ja eine übergeordnete Zusammenfassung der psychischen Symptombelastung darstellen, waren nahezu identisch.

Es lässt sich folgern, dass substanzabhängige Patienten mit sexueller Missbrauchserfahrung eine ähnlich hohe Symptombelastung aufweisen wie schizophrene Patienten. Hier wird deutlich, wie hoch die Intensität der psychischen Probleme bei einer Kombination aus Drogenabhängigkeit und sexueller Missbrauchserfahrung liegt. Diese Erkenntnis ist insbesondere bei einer Gruppenzuordnung der Patienten bezüglich der Therapie der Symptome zu berücksichtigen.

\subsection{Einfluss des Schweregrades des Missbrauchs auf den Krankheitsverlauf}

Eine weitere Kernfrage der Studie befasste sich mit den möglichen Auswirkungen des jeweiligen Missbrauchs unter Berücksichtigung der Einteilung in die drei Schweregrade. So berichteten beispielsweise Sedney und Brooks (1984) sowie Briere und Runtz (1988) in Abhängigkeit vom Schweregrad des sexuellen Missbrauchs über eine erhöhte Rate von Selbstverletzungen und Suizidhandlungen. Briere und Runtz fanden als Folgeerscheinung des Missbrauchs auch Symptome wie Schlafstörungen, Somatisierungs- und dissoziativen Störungen vor. Kendler et al. (2000) stellten fest, dass je härter der Missbrauch, desto höher sei das Risiko eine Bulimie, Alkohol- oder Drogensuchterkrankung zu entwickeln. 
Tabelle 68: Signifikante Composite Scores nach EuropASI bei einer Unterscheidung des Schweregrads des Missbrauchs

\begin{tabular}{|l|l|l|l|l|l|l|}
\hline $\begin{array}{l}\text { Signfikante Composite } \\
\text { Scores nach Europ ASI }\end{array}$ & $\begin{array}{l}\text { Allg. } \\
\text { Missb }\end{array}$ & $\begin{array}{l}\text { Sex. } \\
\text { Missb. }\end{array}$ & $\begin{array}{l}\text { Körp. } \\
\text { Missb. }\end{array}$ & $\begin{array}{l}\text { Vernach- } \\
\text { lässigung }\end{array}$ & $\begin{array}{l}\text { Fam. } \\
\text { Gewalt }\end{array}$ & $\begin{array}{l}\text { Emot. } \\
\text { Missb. }\end{array}$ \\
\hline Körperliche Probleme & & & & & & \\
\hline Arbeit: Ökon. Situation & & & & & & \\
\hline Arbeit: Zufriedenheit & & & & & & \\
\hline Alkoholgebrauch & & & & & & \\
\hline Drogengebrauch & & & & & & \\
\hline Rechtliche Probleme & & & & & & \\
\hline Familie & & & & & & \\
\hline Sozialbeziehungen & & & & & & \\
\hline Psychische Probleme & & & & & & \\
\hline
\end{tabular}

Hellere Schraffierung kennzeichnet signifikante Werte mit $\mathrm{p}<0,05$, dunklere Schraffierung hochsignifikante Werte mit $\mathrm{p}<$ 0,001

Tabelle 69: Signifikante Kennwerte nach SCL 90-R bei einer Unterscheidung des Schweregrads des Missbrauchs

\begin{tabular}{|c|c|c|c|c|c|c|}
\hline $\begin{array}{l}\text { Signfikante Kennwerte nach } \\
\text { SCL } 90-R\end{array}$ & $\begin{array}{l}\text { Allg. } \\
\text { Missb }\end{array}$ & $\begin{array}{l}\text { Sex. } \\
\text { Missb. }\end{array}$ & $\begin{array}{l}\text { Körp. } \\
\text { Missb. }\end{array}$ & $\begin{array}{l}\text { Vernach- } \\
\text { lässigung }\end{array}$ & $\begin{array}{l}\text { Fam. } \\
\text { Gewalt }\end{array}$ & $\begin{array}{l}\text { Emot. } \\
\text { Missb. }\end{array}$ \\
\hline \multicolumn{7}{|l|}{ Somatisierung } \\
\hline \multicolumn{7}{|l|}{ Zwanghaftigkeit } \\
\hline \multicolumn{7}{|l|}{ Unsicherheit Sozialkontakt } \\
\hline \multicolumn{7}{|l|}{ Depressivität } \\
\hline \multicolumn{7}{|l|}{ Ängstlichkeit } \\
\hline \multicolumn{7}{|l|}{ Aggressivität } \\
\hline \multicolumn{7}{|l|}{ Phobische Angst } \\
\hline \multicolumn{7}{|l|}{ Paranoides Denken } \\
\hline \multicolumn{7}{|l|}{ Psychotizismus } \\
\hline \multicolumn{7}{|l|}{$\overline{\text { GSI }}$} \\
\hline \multicolumn{7}{|l|}{ PSDI } \\
\hline PST & & & & & & \\
\hline
\end{tabular}

Hellere Schraffierung kennzeichnet signifikante Werte mit $p<0,05$, dunklere Schraffierung hochsignifikante Werte mit $p<$ 0,001

Auch unsere Ergebnisse zeigten eine Abhängigkeit der Symptome vom 
Schweregrad des Missbrauchs. In Tabelle 68 und Tabelle 69 ist dazu analog zu den vorhergehenden Kapiteln wieder die Übersicht der signifikant gewordenen Kennwerte für die Problembereiche nach EuropASI und die psychischen Probleme nach SCL 90-R zu finden. Insbesondere die Kennwerte der psychischen Probleme bei sexuellem Missbrauch und familiärer Gewalt traten bei einer Unterscheidung des Schweregrads hervor: Fast alle Kennwerte der psychischen Symptombelastungen waren signifikant. Dieses Ergebnis deckte sich, in Bezug auf die Unterteilung des Schweregrads bei sexuellem Missbrauch, mit der Literatur (vgl. Sedney und Brooks 1984, Briere und Runtz 1988). Eine entsprechende Untersuchung in Bezug auf die Auswirkungen des Schweregrades von familiärer Gewalt konnte weder bestätigend noch widersprechend in der Literatur gefunden werden.

Bei körperlichem Missbrauch ergab eine Unterteilung in Schweregrade bei körperlichen Problemen, Sozialbeziehungen und psychischen Problemen signifikante Unterschiede. Keine Unterschiede bezüglich des Schweregrades waren für die Werte des SCL 90-R zu finden. Ähnlich waren die Ergebnisse bei emotionaler Misshandlung.

Eine Unterteilung in Schweregrade war bei einer Vernachlässigung nicht zielführend. Allerdings waren die Fallzahlen für leichte und mittelschwere Vernachlässigung sehr gering, so dass erst eine weiterführende Untersuchung hier Klarheit schaffen könnte. Eine Unterteilung in Männer und Frauen brachte keine Unterschiede.

Zusammenfassend lässt sich sagen, dass bei sexuellem Missbrauch und familiärer Gewalt eine Unterteilung der Schweregrade deutlich mit einer psychischen Symptombelastung der Patienten korrelierte. Bei emotionalem Missbrauch und körperlicher Gewalt waren noch einige Kennwerte bei einer Unterteilung der Schweregrade signifikant. Bei Vernachlässigung brachte eine Kategorisierung nach Schweregrad keine Unterschiede. 


\subsection{Einfluss geschlechtsspezifischer Unterschiede auf den Krankheitsverlauf}

Eine weitere Frage der hier durchgeführten Studie zielt darauf ab, potenzielle geschlechtsspezifische Unterschiede bei der Auswirkung der Traumatisierung auf die Drogenkrankheit zu identifizieren. Dazu wurde jede Missbrauchsgruppe noch einmal in zwei Gruppen je nach Geschlecht unterteilt und mittels statistischer Tests bewertet, ob ein signifikanter Unterschied in den Ergebnissen der Problembereiche nach EuropASI sowie den psychischen Symptomen nach SCL 90-R festzustellen ist. In Tabelle 70 und Tabelle 71 ist wieder die Übersicht über die signifikant gewordenen Kennwerte in Bezug auf die verschiedenen Missbrauchsarten zu finden. Die Kennwerte des EuropASI in Tabelle 70 zeigten dabei keine Unterschiede zwischen den Geschlechtern.

Tabelle 70: Signifikante Composite Scores nach EuropASI bei einer Unterscheidung nach Geschlechtern

\begin{tabular}{|l|l|l|l|l|l|}
\hline $\begin{array}{l}\text { Signfikante Composite } \\
\text { Scores nach Europ ASI }\end{array}$ & $\begin{array}{l}\text { Sex. } \\
\text { Missb. }\end{array}$ & $\begin{array}{l}\text { Körp. } \\
\text { Missb. }\end{array}$ & $\begin{array}{l}\text { Vernach- } \\
\text { lässigung }\end{array}$ & $\begin{array}{l}\text { Fam. } \\
\text { Gewalt }\end{array}$ & $\begin{array}{l}\text { Emot. } \\
\text { Missb. }\end{array}$ \\
\hline Körperliche Probleme & & & & & \\
\hline Arbeit: Ökon. Situation & & & & & \\
\hline Arbeit: Zufriedenheit & & & & & \\
\hline Alkoholgebrauch & & & & & \\
\hline Drogengebrauch & & & & & \\
\hline Rechtliche Probleme & & & & & \\
\hline Familie & & & & & \\
\hline Sozialbeziehungen & & & & & \\
\hline Psychische Probleme & & & & & \\
\hline
\end{tabular}

Hellere Schraffierung kennzeichnet signifikante Werte mit $\mathrm{p}<0,05$, dunklere Schraffierung hochsignifikante Werte mit $\mathrm{p}<$ 0,001 
Tabelle 71: Signifikante Kennwerte nach SCL 90-R bei einer Unterscheidung nach Geschlechtern

\begin{tabular}{|l|l|l|l|l|l|}
\hline $\begin{array}{l}\text { Signfikante Kennwerte nach } \\
\text { SCL 90-R }\end{array}$ & $\begin{array}{l}\text { Sex. } \\
\text { Missb. }\end{array}$ & $\begin{array}{l}\text { Körp. } \\
\text { Missb. }\end{array}$ & $\begin{array}{l}\text { Vernach- } \\
\text { lässigung }\end{array}$ & $\begin{array}{l}\text { Fam. } \\
\text { Gewalt }\end{array}$ & $\begin{array}{l}\text { Emot. } \\
\text { Missb. }\end{array}$ \\
\hline Somatisierung & & & & & \\
\hline Zwanghaftigkeit & & & & & \\
\hline Unsicherheit Sozialkontakt & & & & & \\
\hline Depressivität & & & & & \\
\hline Ängstlichkeit & & & & & \\
\hline Aggressivität & & & & & \\
\hline Phobische Angst & & & & & \\
\hline Paranoides Denken & & & & & \\
\hline Psychotizismus & & & & & \\
\hline GSI & & & & & \\
\hline PSDI & & & & & \\
\hline PST & & & & & \\
\hline
\end{tabular}

Hellere Schraffierung kennzeichnet signifikante Werte mit $\mathrm{p}<0,05$, dunklere Schraffierung hochsignifikante Werte mit $\mathrm{p}<$ 0,001

Beim sexuellen Missbrauch konnte kein signifikanter Unterschied in den Problembereichen nach EuropASI und dem psychischen Zustand nach SCL 90$\mathrm{R}$ zwischen den männlichen und weiblichen drogenkranken Missbrauchsopfern festgestellt werden. Bei der Untersuchung der Unterschiede für die Gruppe mit körperlicher Misshandlung in der Kindheit wurde ein Kennwert signifikant. Die Skala „Unsicherheit im Sozialkontakt“" wies für die Frauen einen signifikant schlechteren Wert auf. Dies könnte darauf hindeuten, dass die Traumatisierung das Selbstwertgefühl der Frauen stärker beeinflusst. Die dritte Missbrauchsgruppe hatte unter einer Vernachlässigung in der Kindheit zu leiden. Hier zeigten sich geschlechtsspezifische Unterschiede bei der „Phobischen Angst“. Frauen tendieren demnach bei einer Missbrauchserfahrung durch Vernachlässigung vermehrt zu Furchtattacken. Bei einem Missbrauch durch familiäre Gewalt war kein Unterschied zwischen Männern und Frauen zu finden. Schließlich erfolgte die Analyse noch für die Gruppe mit emotionalem Missbrauch auf potenzielle Unterschiede zwischen Männern und Frauen. Hier waren zwei 
Skalenwerte signifikant: „Unsicherheit im Sozialkontakt“ und „phobische Angst“. Bei beiden wiesen die Frauen die schlechteren Werte auf.

Unsere Ergebnisse bestätigen, was Karren-Derber (1989) und Salter (1988) für die unterschiedlichen Coping- und Verarbeitungsmechanismen von Frauen und Männern nach Missbrauchserfahrungen beschrieben haben: Männer entwickeln nach Missbrauchserfahrungen eher externalisierende Verarbeitungsformen, d.h. nach außen gerichtete Aggressionen. Frauen reagieren internalisierend auf das Missbrauchstrauma, d.h. nach innen gerichtete Aggressionen in Form von Angst, Selbstzweifeln und Schuldgefühlen überwiegen. Suchtmittelgebrauch scheint gerade bei Frauen, die in der Kindheit missbraucht und misshandelt worden sind, zum Problem zu werden. Sie greifen vermehrt zu Mitteln, die ihnen kurzfristig Entspannung, Angst- und Bewusstseinsminderung, aber auch Abbau von Schuldgefühlen verschaffen und einen Lösungsweg darstellen, mit Angst, Depressionen und Selbstzweifeln besser umgehen zu können (vgl. KarrenDerber, 1989 und Salter, 1988). Auch Kreyssig (1997) schließt sich dieser Meinung an.

Zusammenfassend lässt sich sagen, dass der vorgenommene Vergleich nur in wenigen Bereichen einen Unterschied zwischen Männern und Frauen in den analysierten Belastungen nach einer Missbrauchserfahrung aufwies. Überhaupt in Erscheinung traten lediglich die psychischen Symptome in den Skalen „Unsicherheit im Sozialkontakt“ und „phobische Angst“. Drogenkranke Frauen zeigten unter den Folgen der oben genannten Traumatisierungen somit eher ein reduziertes Selbstwertgefühl und ein höheres Angstpotenzial als männliche Patienten. 


\subsection{Ansatz einer Gruppenbildung unter Berücksichtigung der Missbrauchsart, des Schweregrads und des Geschlechts für eine differenzierte Therapie}

Zur systematischen Eingliederung sowie zum Erlangen differenzierter Behandlungsstrategien wird in der Literatur eine Bildung von Untergruppen bezüglich komorbider psychischer Störungen, Substanzmissbrauch und Realtraumatisierung vorgeschlagen (vgl. Krausz und Müller-Thomsen 1994, Krausz und Freyberger 1997). Diese Untergruppen konzentrieren sich auf die spezielle Situation der Symptombelastung und Therapieerfordernisse von Patienten mit einer kindlichen Traumatisierung. Rounsaville et al. (1982) beschrieb für die Gruppe mit zusätzlich feststellbarem Kindheitstrauma gegenüber primär Süchtigen und primär straffälligen Süchtigen die schlechtesten therapeutischen Chancen. Auch Schäfer et al. (2000) berichteten, dass die Auswirkungen des sexuellen Missbrauchs in der Kindheit sich eher durch einen ungünstigen Verlauf und schlechtere Prognose der späteren Suchterkrankung zeigten.

Leider liegt eine Differenzierung bezüglich der fünf Missbrauchsarten, der Missbrauchsschwere, der Aufteilung und Intensität der Symptombelastung, wie wir sie in unserer Studie vorgenommen haben, nicht in vergleichbarer Form in der Literatur vor. Aus diesem Grunde werden wir im folgenden in Abhängigkeit dieser Kriterien einen eigenen Vorschlag für die Gruppenbildung entwickeln.

Generell zeigte sich in unserer Untersuchung, dass die Missbrauchsgruppen bei den betrachteten Problembereichen nach EuropASI nur eine geringe Anzahl an signifikanten Unterschieden bezüglich den Belastungen aufwies. Diese waren hauptsächlich in den Bereichen „psychische“ und „körperliche Probleme“. Die Auswirkungen einer Traumatisierung in der Kindheit auf die betrachteten Problembereiche ist nach EuropASI als gering bis mittel einzustufen. 
Dagegen macht eine Analyse der psychischen Probleme mit SCL 90-R deutlich, dass die Missbrauchsgruppen sich in einer Vielzahl der Symptome signifikant von der Vergleichsgruppe ohne den jeweiligen Missbrauch abheben. Hier ist von einer starken Belastung der Drogenpatienten durch die Traumatisierung in ihrer Kindheit auszugehen.

Es soll nun der Versuch unternommen werden, die Ergebnisse zu den einzelnen Missbrauchsgruppen grob nach der Stärke ihrer Auswirkungen zu gliedern. Dies kann auf Grund der großen Anzahl der gemessenen Parameter und deren struktureller Unterschiede (verschiedene signifikante Problembereiche und Nichtvergleichbarkeit der psychischen Symptome miteinander, signifikante Werte vs. hochsignifikante Werte, Kennwerte des EuropASI im Vergleich zu SCL 90-R, Ergebnisse der Analyse der Mittelwerte) nicht in einem direkten Vergleich, sondern nur in einem groben Raster erfolgen. Trotzdem erscheint diese Art der Rasterung bei den vorliegenden Ergebnissen als möglich und sinnvoll. Als Raster wurden sowohl die Anzahl der signifikanten und hochsignifikanten Symptome, als auch die Mittelwerte der Problembereiche (EuropASI) und psychischen Symptome (SCL 90-R) herangezogen.

Aus den Analysen der Art und Intensität der Symptombelastungen ergab sich, dass die drogenkranken Patienten, die eine sexuelle Missbrauchserfahrung erlitten haben, am stärksten von den Auswirkungen einer Traumatisierung in der Kindheit betroffen sind (höchste Mittelwerte bei psychischen Symptomen, hohe Anzahl an signifikant schwereren psychischen Symptomen). Am zweitstärksten hatte die Gruppe mit Vernachlässigung mit den Folgen ihrer Traumatisierung zu kämpfen (zweithöchste Mittelwerte bei psychischen Symptomen, höchste Anzahl an signifikant stärkeren Problembereichen, höchste Anzahl an signifikant schwereren psychischen Symptomen). Danach kam die Gruppe mit familiärer Gewalt (zweithöchste Anzahl an signifikant schweren psychischen Symptomen, zweithöchste Mittelwerte bei sieben von neun psychischen Symptomen). Dieser 
folgte die Gruppe mit emotionalem Missbrauch (mittlere Anzahl an signifikant schweren psychischen Symptomen, zweithöchste Anzahl an signifikant stärkeren Problembereichen). Zuletzt war die Gruppe mit körperlicher Missbrauchserfahrung zu nennen (niedrige Anzahl an signifikant stärkeren Problembereichen, niedrigste Anzahl an signifikant schwereren psychischen Symptomen).

Berücksichtigt man noch die Ergebnisse der Analyse des Schweregrades des jeweiligen Missbrauchs aus Kapitel 4.2, so ergibt sich folgende Reihenfolge:

- Sexueller Missbrauch mit Unterscheidung in leichten \& mittleren Schweregrad versus schweren Missbrauch

- Vernachlässigung ohne Unterscheidung des Schweregrades

- Familiäre Gewalt mit Unterscheidung in leichten \& mittleren Schweregrad versus schweren Missbrauch

- Emotionaler Missbrauch mit Unterscheidung in leichten \& mittleren Schweregrad versus schweren Missbrauch

- Körperliche Misshandlung ohne Unterscheidung des Schweregrades

- Kein Missbrauch

Eine Kategorisierung in Männer und Frauen lag auf Grund der sehr geringen Unterschiede nicht nahe. Lediglich die zwei signifikant gewordenen Symptombelastungen „Unsicherheit im Sozialkontakt“ und „phobische Angst“ sind zu bedenken. Allgemeiner Missbrauch wurde in dieser Einteilung außen vor gelassen, da es sich hier nur um eine Zusammenfassung verschiedenster Missbrauchsformen handelt, die differenzierter in die obige Einteilung eingehen. Aus unseren Daten ging außerdem hervor, dass sich Einzel- und Gruppentherapien insbesondere auf die in Tabelle 63 und Tabelle 64 aufgezeigten signifikant schwereren Symptome der einzelnen Missbrauchsgruppen konzentrieren sollten.

Auf der Basis unserer Ergebnissen sowie den Studier von Krausz und Müller- 
Thomsen (1994), Krausz und Freyberger (1997) und Schäfer et al. (2000) empfehlen wir, eine Gruppeneinteilung bei der Therapie von drogenkranken Patienten mit Missbrauchserfahrung heranzuziehen. Sie ermöglicht es, auf die spezifischen und unterschiedlich schweren Symptombelastungen einzugehen. Insbesondere die Berücksichtigung der jeweiligen Missbrauchserfahrung in der Therapie stellt aus unserer Sicht einen Schlüssel bei der Lösung der psychischen Probleme und der Drogenabhängigkeit der Patienten dar.

\section{5 Überprüfung der Stichprobe und Methodenkritik}

Im Folgenden wird ein Vergleich der bei uns gefundenen Prävalenz zu sexuellem Missbrauch, körperlicher Misshandlung, Vernachlässigung, emotionaler Misshandlung und familiärer Gewalt mit den Ergebnissen der Literatur durchgeführt. Damit soll die Vorgehensweise bezüglich der Auswahl der Stichprobe überprüft werden.

\subsubsection{Vergleich der Zusammensetzung dieser Stichprobe mit der Literatur}

Unsere 83 Patienten waren zum Zeitpunkt der Erhebung durchschnittlich 30 Jahre alt. An der Befragung nahmen 34\% Frauen und 66\% Männer teil. Diese Verteilung deckt sich mit den in der Literatur zu findenden durchschnittlichen Werten. Flügel und Merfert-Diete (1993) beschreiben, dass nach Quellen der deutschen Hauptstelle gegen die Suchtgefahren (DHS) der Frauenanteil unter den von illegalen Drogen Abhängigen (nach dem Betäubungsmittelgesetz) bei ca. einem Drittel liegt. Wallen (1992) nennt in seiner Untersuchung an 229 Heroinsüchtigen einen Frauenanteil von $21 \%$.

Dies indiziert, dass die vorliegende Studie repräsentativ für die geschlechtsspezifische Verteilung ist. Die Männer waren im Schnitt 21 Jahre alt, die Frauen ca. 28 Jahre. Dies deckt sich ebenfalls mit den Angaben von Flügel 
und Merfert-Diete (1993).

\subsubsection{Vergleich der Ergebnisse zur Prävalenz von Kindesmissbrauch in der Literatur}

\section{Sexueller Missbrauch}

Zunächst wird ein Auszug aus Studien zur Prävalenz von sexuellem Missbrauch in der Normalbevölkerung vorgestellt. Die Prävalenz in der Normalbevölkerung benötigen wir zum Vergleich mit den Ergebnissen unserer Studie. Eine Untersuchung von Schrötensack et al. (1991) bei 1841 Berufsschülern und Studenten im Alter von 20 bis 25 Jahren in Würzburg erbrachte einen Missbrauchsanteil überhaupt mit 5,8\% der Männer und 16,1\% der Frauen. Ein Missbrauch mit Körperkontakt ergab sich dabei bei 4\% der Männer und 10,3\% der Frauen. Sie erhoben auch das Verhältnis missbrauchter Jungen zu Mädchen. Sie stellten fest, dass es bei 1: 2 bis 1: 4 lag. Raupp \& Egger (1993) gaben die Prävalenz für sexuellen Missbrauch bei Männern mit 6\% an. Auch Wallen (1992) fand mit $8 \%$ ebenfalls keine wesentlich andere Prävalenz in der Normalbevölkerung. Bange (1992) befragte 518 weibliche und 343 männliche Studierende in Dortmund zu einer sexuellen Missbrauchsvorgeschichte. Dabei gaben $25 \%$ der Frauen und $8 \%$ der Männer an, sexuell missbraucht worden zu sein, hiervon 12\% der Frauen und mehr als die Hälfte der Männer „schwer“. Eine Untersuchung von Fischer zeigte bei ca. 30\% der Frauen und 10\% der Männer einen unfreiwilligen sexuellen Kontakten vor dem 18. Lebensjahr. Bei dieser Frage wurde allerdings die weiteste Definition von sexuellem Missbrach in Kindheit und Jugend zugrunde gelegt, indem alle Varianten von sexuellem Kontakt einbezogen sind sowie familiäre und außerfamiliäre Vorfälle gleichermaßen eingehen (Fischer 1998). Eine Untersuchung von MacMillan et al. (1997) fand mit 9953 Probanden in Kanada (Ontario) statt. Sexueller Missbrauch wurde bei 12,8\% der Frauen und 4,3\% der Männer gefunden, wobei in 11,1\% bei Frauen und in 3,9\% bei Männern ein schwerer Missbrauch angegeben wurde. In der Hamburger Studie (Richter-Appelt, 1997) wurden mittels eines Fragebogens 
1085 Hamburger Studenten und Studentinnen zu Missbrauchserfahrungen in der Kindheit (vor dem 12. Lebensjahr) befragt. Insgesamt gaben 23\% der Frauen und $4 \%$ der Männer an, sexuell missbraucht worden zu sein. Aus den Studien von Kreyssig (1997) geht eine Prävalenz für sexuellen Missbrauch bei Frauen von $10 \%$ bis $15 \%$ in der Normalbevölkerung hervor. Welch und Fairburn führten 1994 ein Fall-Kontrollstudie durch. Sie untersuchten nach einem standardisierten DSM-III-R Verfahren 50 Bulimiekranke, 50 Frauen mit anderen psychischen Erkrankungen und 100 Frauen ohne psychiatrische Diagnose. Dabei ergab sich bei Frauen ohne psychiatrische Diagnose eine Prävalenz für sexuellen Missbrauch in der Kindheit von 10\%.

Es ist ersichtlich, dass die Ergebnisse für die Prävalenz von sexuellem Missbrauch in den Studien variieren. Dies ist zum einen auf unterschiedliche Methoden bei der Erhebung zurückzuführen, andererseits aber auch auf die schon diskutierte Schwierigkeit bei der Definition des sexuellen Missbrauchs. Sehr umfassend hat Finkelhor (1997) eine Vielzahl der Studien analysiert und die Ergebnisse in einer Übersicht zusammengestellt, die in der Literatur sehr häufig als Basis verwendet wird und auch bei uns als Vergleichsgrundlage dienen soll:

- Alle Untersuchungen erbrachten sexuelle Missbrauchserfahrungen bei wenigstens $7 \%$ der Frauen und 3\% der Männer

- 1,5 bis 3 mal höhere Rate der Opfer bei Frauen als bei Männern

- Alle Studien, die Langzeiteffekte untersuchten, zeigten, dass ein sexueller Missbrauch mit Störungen der psychischen Gesundheit im Erwachsenenalter einherging

Auch wenn man nur die schweren Missbrauchsfälle zugrunde legt, so erlitten in unserer Studie ca. drei bis vier mal so viele Patienten einen sexuellen Missbrauch in der Kindheit wie die hier aufgeführten Vergleichsstichproben aus der Normalbevölkerung. Zählt man die mittelschweren Missbrauchsfälle dazu leichte sexuelle Missbrauchsfälle traten in unserer Studie nicht singulär auf -, dann erhöht sich das Verhältnis gegenüber der Normalbevölkerung um den 
Faktor sieben bis acht.

Außerdem haben wir den Vergleich mit Ergebnissen von Studien zur Prävalenz von sexuellem Missbrauch unter Drogensüchtigen angestellt. Mit 33\% (57\% der Frauen und $20 \%$ der Männer) lag der Anteil der sexuell missbrauchten drogensüchtigen Patienten in unserer Studie unter den Angaben von Russell (1983) und Rush (1985). Bei einer Untersuchung von drogenabhängigen Frauen fand Russell eine Prävalenz für sexuellen Missbrauch von $80 \%$. In der Kritik stand allerdings die von Russell verwendete Definition von sexuellen Missbrauch, welche sehr weit gefasst war (vgl. auch Kapitel 2.2.1, Definition von sexuellem Missbrauch). Ähnliche Ergebnisse erhielt auch Rush (1985) bei einer Befragung von weiblichen Drogenabhängigen. Er berichtet, das $80 \%$ der drogenabhängigen Frauen in ihrer Kindheit sexuell missbraucht worden sind.

Kreyssing (1997) bezifferte den Anteil sexuell Missbrauchter an allen drogenabhängigen Frauen zwischen 60\% und 80\%. Bei Arenz-Greiving (1994) gaben von 1054 befragten drogenabhängigen Frauen $30 \%$ einen sexuellen Missbrauch vor ihrem 16. Lebensjahr an.

Beutel führte 1999 eine Fragebogenstudie mit 470 suchtkranken Patientinnen und einer Kontrollgruppe mit 181 Frauen durch. Die suchtkranken Frauen wurden in ihrer Kindheit signifikant häufiger missbraucht (24,5\%) als Frauen, die später nicht suchtkrank wurden (10,2\%). Berücksichtigt man die engere Definition für sexuellen Missbrauch, so zeigt sich eine Übereinstimmung unserer Ergebnisse für die Stichprobe der schwer missbrauchten Frauen mit den Ergebnissen von Beutel.

Auch hier wird deutlich, dass die Werte in der Literatur für die Prävalenz von sexuellem Missbrauch bei Drogenabhängigen in Abhängigkeit der zu Grunde gelegten Systematik stark schwanken. Die bei uns gefundenen Werte für die Prävalenz von sexuellem Missbrauch bewegten sich dabei im Mittelfeld der 
Ergebnisse der Studien. Von einer plausiblen Messung der Werte für sexuellen Missbrauch ist folglich auszugehen.

\section{Körperliche Misshandlung}

Bezüglich der Prävalenz von körperlicher Misshandlung in der Normalbevölkerung gab Engfer die Zahl der Eltern, die ihre Kinder körperlich bestrafen mit $50 \%$ bis $75 \%$ an. Schwerwiegendere und relativ häufige Bestrafungen, die als körperlicher Missbrauch aufzufassen sind und bei uns in die Kategorie mittelschwere bis schwere körperliche Misshandlung einzuordnen sind, lagen nach Engfer bei 10\%15\% (Engfer 1991, 1997, Esser 1994). Eine Studie von MacMillan et al. (1997) ermittelte unter 9953 Befragten in Kanada (Ontario) bei Männern in 31,2\% und bei Frauen in $21,1 \%$ körperliche Misshandlungen im Kindesalter. Coleman et al. (1995) beschrieben die Inzidenz für körperliche Gewalt mit 36,7\%. In der Hamburger Studie von Richter-Appelt (1997) gaben 28\% der befragten Frauen und 14\% der Männer an, körperlich misshandelt worden zu sein.

Simpson et al. (2002) berücksichtigten in ihrer Analyse 126 Studien, die den Zusammenhang zwischen sexuellem und körperlichem Missbrauch in der Kindheit und einer späteren Suchterkrankung behandelten. Ausgehend von einer vorliegenden Drogenabhängigkeit fanden sie bei Frauen eine ca. doppelt so hohe Rate an körperlichen Missbrauchsopfern (46\%) verglichen mit den von MacMillan et al. (1997) gefundenen Referenzwerten in der Normalbevölkerung (21\%). Bei den drogenabhängigen Männern war keine erhöhte Rate an körperlichen Missbrauchsopfern gegenüber der Normalbevölkerung zu verzeichnen.

Eine körperliche Misshandlung fand sich in unserer Stichprobe bei 54\% unserer Patienten. Dabei waren 61\% der Frauen und 51\% der Männer betroffen, ohne dass der Unterschied signifikant wurde. Unsere Ergebnisse decken sich für Frauen mit den oben beschriebenen Werten von Simpson et al. (2002). Ebenso interessant ist eine Betrachtung der Prävalenz unter Berücksichtigung des 
Schweregrades des körperlichen Missbrauchs. Insgesamt waren bei uns rund ein Drittel der Männer und 29\% der Frauen von schwerem körperlichen Missbrauch in der Kindheit betroffen. Legt man die Ergebnisse von Engfer zu Grunde so ist für eine schwere körperliche Misshandlung eine ca. zwei bis drei mal höhere Prävalenzrate in unserer Studienpopulation gegenüber der Normalbevölkerung vorzufinden. Malinowsky-Rummel \& Hansen (1993) untersuchten in einer Stichprobe von Dogenabhängigen die Prävalenz für körperlichen Missbrauch. Dabei variierte die Prävalenz in einer Spanne von 13\% bis 35\%. Als langfristige Folgen der körperlichen Misshandlungen traten gehäuft Alkohol- und Drogenmissbrauch (v.a. bei misshandelten Männern), emotionale Labilität, erhöhte Aggressionsbereitschaft und erhöhte Suizidneigung (v.a. bei misshandelten und missbrauchten Frauen) auf. In dem von Malinowsky-Rummel \& Hansen beschriebenen Bereich liegen auch die Ergebnisse von Wallen (1992), der einen körperlichen Missbrauch sowohl bei 30\% der drogenabhängigen Männer, als auch der Frauen nachwies. Diese Angaben decken sich mit den bei uns gefundenen Werten für die Prävalenz von schwerem körperlichen Missbrauch. Von einer plausiblen Messung der Werte für körperliche Misshandlung ist folglich auszugehen.

\section{Vernachlässigung}

Nach Besharov (1993) lagen in den Vereinigten Staaten im Jahr 1986 ca. 700.000 Fälle mit Vernachlässigung und 140.000 Fälle mit sexuellem Missbrauch vor. Dies entspricht einem Verhältnis von 5:1. Nimmt man die Prävalenzraten für sexuellen Missbrauch nach Finkelhor (1997) mit 3\%-7\%, so ergäbe sich daraus geschätzt eine Prävalenzrate für Vernachlässigung zwischen $15 \%$ und $35 \%$ in der Normalbevölkerung.

In unserer Studie gaben 79\% der Frauen und 62\% der Männer Vernachlässigung in der Kindheit an. Insgesamt zeigte sich eine Vernachlässigung bei $67 \%$ der Befragten. Unter schwerer Vernachlässigung litten 57,8\% der Befragten in ihrer 
Kindheit. Die Frauen wiesen in knapp zwei Drittel der Fälle schwere Vernachlässigung in der Kindheit auf. Unsere Werte überstiegen auf Grund der Stichprobe unter drogenabhängigen Patienten die obige Schätzung gegenüber der Normalbevölkerung deutlich. Legt man das Verhältnis zwischen der Prävalenz in der Normalbevölkerung und bei suchtkranken Patienten von sexuellem Missbrauch zu Grunde, so ergibt sich ein Faktor größer vier. Damit lägen unsere Werte für die Prävalenz von Vernachlässigung bei suchtkranken Patienten innerhalb dieses geschätzten Bereichs.

\section{Familiäre Gewalt}

Bei familiärer Gewalt lagen die Angaben der Patienten in unserer Befragung bei $51 \%$. Die geschlechtsspezifische Aufteilung ergab $47 \%$ bei den Männern und $57 \%$ bei den Frauen. Entsprechende schwere Gewalterfahrungen konnten 13\% der Männer und 22\% der Frauen zugeordnet werden. Eine Gegenüberstellung unserer Ergebnisse zur Literatur, wie dies bei sexuellem Missbrauch und körperlicher Misshandlung erfolgt ist, war nicht möglich, da eine differenzierte Aufteilung nach familiärer Gewalt in den Studien so nicht vorliegt.

\section{Emotionale Misshandlung}

Eine emotionale Misshandlung erlitten 66\% der Studienpopulation. Dabei waren 65\% der Männer und 67\% der Frauen betroffen. Knapp 28\% waren mit dieser Missbrauchserfahrung schwer belastet. 32\% der Frauen wiesen Erfahrungen mit schwerem emotionalen Missbrauch auf. Bei den Männern zeigten rund ein Viertel den höchsten Schweregrad an emotionalem Missbrauch. Eine Gegenüberstellung unserer Ergebnisse zur Literatur, wie dies bei sexuellem Missbrauch und körperlicher Misshandlung erfolgt ist, war ebenfalls nicht möglich, da keine differenzierte Aufteilung nach emotionalem Missbrauch in den Studien gefunden werden konnte. 


\subsection{3 Überprüfung der Ergebnisse des SCL 90-R durch einen Vergleich mit Referenzwerten von Franke}

Die Plausibilität unserer Studie sollte außerdem durch einen Vergleich unserer Ergebnisse mit SCL 90-R mit den Studienergebnissen des Urhebers des SCL 90 - R, G.H. Franke, überprüft werden.

Franke (1995) hatte in seinen Studien u.a. für substanzabhängige Patienten die Skalenwerte und globalen Kennwerte erfasst (siehe Spalte „Sub. Abh.“ in Tabelle 73). Diese wollten wir nun mit unseren Ergebnissen nach SCL 90-R unserer substanzabhängigen Patientenstichprobe vergleichen (siehe dazu Spalte „Ges“ für die Ergebnisse nach SCL 90-R über alle unserer Patienten in Tabelle 72).

Tabelle 72: Vergleich der Mittelwerte der Skalenwerte für die verschiedenen Missbrauchsgruppen, die Gruppe ohne Missbrauch und die gesamte Stichprobe

\begin{tabular}{|c|c|c|c|c|c|c|c|c|}
\hline $\begin{array}{l}\text { Skalenwerte nach } \\
\text { SCL 90-R (MW) }\end{array}$ & Allg. & Sex. & Körp. & Vern. & $\begin{array}{l}\text { Fam. } \\
\text { Gew. }\end{array}$ & Emo. & Kein & Ges. \\
\hline Somatisierung & 1,05 & 1,20 & 1,09 & 1,14 & 1,18 & 1,11 & 0,59 & 0,97 \\
\hline Zwanghaftigkeit & 1,21 & 1,44 & 1,22 & 1,31 & 1,31 & 1,22 & 0,88 & 1,15 \\
\hline $\begin{array}{l}\text { Unsicherheit } \\
\text { Sozialkontakt }\end{array}$ & 0,95 & 1,29 & 0,99 & 0,98 & 1,09 & 0,98 & 0,55 & 0,87 \\
\hline Depressivität & 1,36 & 1,74 & 1,39 & 1,44 & 1,52 & 1,40 & 0,82 & 1,26 \\
\hline Ängstlichkeit & 1,19 & 1,36 & 1,26 & 1,31 & 1,22 & 1,22 & 0,55 & 1,07 \\
\hline Aggressivität & 1,09 & 1,20 & 1,11 & 1,15 & 1,13 & 1,10 & 0,80 & 1,03 \\
\hline Phobische Angst & 0,53 & 0,72 & 0,55 & 0,60 & 0,65 & 0,56 & 0,16 & 0,46 \\
\hline $\begin{array}{l}\text { Paranoides } \\
\text { Denken }\end{array}$ & 1,03 & 1,31 & 1,14 & 1,08 & 1,19 & 1,09 & 0,56 & 0,95 \\
\hline Psychotizismus & 0,62 & 0,89 & 0,65 & 0,68 & 0,72 & 0,69 & 0,19 & 0,54 \\
\hline GSI & 1,04 & 1,29 & 1,08 & 1,12 & 1,16 & 1,08 & 0,59 & 0,96 \\
\hline PSDI & 2,01 & 2,10 & 2,04 & 2,05 & 2,05 & 2,04 & 1,63 & 1,95 \\
\hline PST & 43,1 & 51,0 & 44,0 & 45,9 & 47,2 & 43,8 & 28,8 & 40,5 \\
\hline
\end{tabular}


Tabelle 73: Referenzwerte nach Franke für Normal Gesunde, HIV infizierte, substanzabhängige und schizophrene Patienten

\begin{tabular}{lllll}
\hline $\begin{array}{l}\text { Skalenwerte nach } \\
\text { SCL 90-R (MW) }\end{array}$ & Norm & HIV & $\begin{array}{l}\text { Sub. } \\
\text { Abh. }\end{array}$ & Schiz. \\
\hline Somatisierung & 0,35 & 0,79 & 0,74 & 1,11 \\
\hline Zwanghaftigkeit & 0,47 & 0,85 & 1,15 & 1,45 \\
\hline $\begin{array}{l}\text { Unsicherheit } \\
\text { Sozialkontakt }\end{array}$ & 0,41 & 0,72 & 0,86 & 1,22 \\
\hline Depressivität & 0,40 & 1,00 & 1,23 & 1,38 \\
\hline Ängstlichkeit & 0,29 & 0,78 & 1,13 & 1,33 \\
\hline Aggressivität & 0,31 & 0,60 & 0,61 & 0,91 \\
\hline Phobische Angst & 0,14 & 0,46 & 0,75 & 1,08 \\
\hline Paranoides Denken & 0,35 & 0,65 & 0,69 & 1,42 \\
\hline Psychotizismus & 0,18 & 0,59 & 0,57 & 1,18 \\
\hline GSI & 0,33 & 0,76 & 0,91 & 1,24 \\
\hline PSDI & 1,22 & 1,58 & - & 2,11 \\
\hline PST & 23,10 & 39,48 & - & 50,78 \\
\hline
\end{tabular}

Grau hinterlegt sind die vergleichbaren Werte mit den Ergebnissen unserer Studie

Das Ergebnis dieses Vergleichs zeigte eine sehr hohe Übereinstimmung der von uns gefundenen Skalenwerte mit denen von Franke: Die Skalen Zwanghaftigkeit, Unsicherheit im Sozialkontakt, Depressivität, Ängstlichkeit und Psychotizismus waren bis auf eine Abweichung von höchstens 0,05 identisch! Ebenso zeigte der globale Kennwert nur eine Abweichung von 0,05. Abweichungen mit etwas größeren Werten gegenüber unserer Stichprobe gab es nur bei Somatisierung, Aggressivität und paranoidem Denken. Abweichungen mit niedrigeren Werten gegenüber unserer Stichprobe lag lediglich bei phobischer Angst vor.

Durch diesen Vergleich wurde gezeigt, dass unsere substanzabhängige Patientenstichprobe sehr ähnliche Werte wie die von Franke erfasste substanzabhängige Patientenstichprobe erbrachte. Entsprechend kann durch diese weitgehende Übereinstimmung in den Skalenwerten sowie beim globalen Kennwert von einer plausiblen Stichprobe und Messung in unserer Studie ausgegangen werden. 


\subsubsection{Methodenkritik}

Prinzipiell liegen bei der Erhebung mit Fragebögen und Interviews Risiken der Verfälschung des Ergebnisses vor. Erhebungstechniken, bei denen sich die Untersuchungsteilnehmer bewusst darüber sind, dass sie Gegenstand einer Untersuchung sind und mit ihren Antworten die Möglichkeit haben auf Messergebnisse Einfluss zu nehmen, unterliegen diesem Einflussfaktor. Sowohl Reaktionen auf die Art der Fragenstellung, als auch auf den Interviewer, können zu Antwortverzerrungen führen und damit die Validität gefährden. Insbesondere bei der Auswertung der Fragen, die auf einer Selbsteinschätzung der Patienten beruhen, sind als Formen der Antwortverzerrung vor allem die Selbstdarstellung, die soziale Erwünschtheit und die Antworttendenzen zu bedenken. Die Selbstdarstellung ist ein, bei jeder Art von Kommunikation präsentes Phänomen, welches per se nicht obligat einen Störfaktor darstellt.

\section{EuropASI}

Bei dem EuropASI-Interview werden die Untersuchungsteilnehmer nicht nur zu ihrer Drogenproblematik, sondern auch zu Problemen im beruflichen, rechtlichen, sozialen, familiären, körperlichen und psychischen Bereich befragt, worüber sie sich möglicherweise vorher noch keine Gedanken gemacht haben. Die Patienten sind aufgefordert, zu den angesprochenen Themen Stellung zu nehmen und reflektieren mehr oder weniger bewusst, was sie mitteilen wollen und was nicht, wem die Informationen zukommen, wie der Interviewer reagieren könnte und welche Konsequenzen für sie daraus entstehen könnten. Die Angst vor nicht gewahrter Anonymität ist insbesondere im rechtlichen Bereich zu berücksichtigen, da die Frage nach illegalen Tätigkeiten zum Teil entweder abgelehnt oder vielleicht auch unehrlich beantwortet wurde. Eigene Verhaltensweisen als widersprüchlich oder unvernünftig zu erkennen ist unangenehm, so dass Falschantworten eher der Selbsttäuschung als der Fremdtäuschung dienen. Bewusst positiv gefärbte Selbstdarstellungen könnten 
auch im Sinne einer Zukunftsprognose zu interpretieren sein (Bortz, Döring, 1995). So kann z. B. die positive Bewertung einer Stresssituation eher einem Wunschdenken als der Realität entsprechen. Dies alles kann dazu führen, dass die Ergebnisse verfälscht werden.

Dennoch wird der EuropASI als eine der besten Erhebungsmethoden angesehen. Eine hohe Durchführungsobjektivität wird beim EuropASI durch standardisierte Instruktionen mit Hilfe des „Manual für Training und Durchführung von Interviews mit dem EuropASI" erreicht. Auswertungs- und Interpretationsobjektivität sind durch die Art der Itemformulierung, die weitgehend vorgegebenen Antwortmöglichkeiten und vor allem durch die Auswertung mit Hilfe des EuropASI Composite Scores gegeben. Die Reliabilität der deutschen Übersetzung des EuropASI wurde verschiedentlich nachgewiesen. In einer Untersuchung von Scheurich et al. wiesen die Subskalen keine oder nur eine leichte Korrelation auf. Die interne Konsistenz lag bei Werten von 0,69 bis 0,92 nach Cronbach (siehe in Scheurich et. al. 2000). Die Validität konnte ebenfalls nachgewiesen werden (Scheurich et al. 2000).

\section{SCL 90-R}

Das Kriterium der Objektivität erfüllt der SCL 90-R durch seinen Aufbau und sein standardisiertes Auswerteverfahren Die Objektivität eines Tests gibt an, in welchem Ausmaß die Testergebnisse vom Testanwender unabhängig sind. Eine hohe Durchführungsobjektivität wird durch die standardisierten Instruktionen erreicht. Auswertungs- und Interpretationsobjektivität sind durch die Art der Itemformulierung, die vorgegebenen Antwortmöglichkeiten und durch die standardisierte Auswertung gegeben.

Die Reliabilität misst die Zuverlässigkeit, bei Wiederholung gleiche Ergebnisse zu erzielen. Bezüglich der Reliabilität des $\mathrm{SCL}$ 90-R berichtet Franke für verschiedene klinische und nicht-klinische Stichproben folgende Werte (Cronbach's alpha): In einer gesunden Vergleichsgruppe $(n=1006)$ lag die 
interne Konsistenz zwischen 0,51 (Skala Phobische Angst) und 0,83 (Skala Depressivität) sowie 0,94 (GSI). In einer klinischen Stichprobe ( $n=568)$ lag die interne Konsistenz zwischen 0,78 (Paranoides Denken), 0,79 (Aggressivität/Feindseligkeit, Phobische Angst) und 0,89 (Depressivität) sowie 0,98 (GSI) (Franke, 1992).

Validität (auch Gültigkeit) bezieht sich auf die Frage, ob ein Item (eine Skala, ein Test, ein Fragebogen) das misst, was es zu messen vorgibt. Die faktorielle Validität der Originalfaktorenstruktur des SCL 90-R konnte in verschiedenen Stichproben nachgewiesen werden (Franke et. al. 2000, Franke 1995).

Der SCL 90-R wird heute sowohl im Bereich der klinischen Diagnostik als auch zur Veränderungsmessung im Rahmen von Therapie und Forschung als ökonomisches, reliables und valides Instrument eingesetzt.

Insbesondere der Vergleich und die Übereinstimmung unserer Skalenwerte des SCL 90-R für drogenabhängige Patienten, mit denen von Franke für drogenabhängige Patienten (vgl. Kapitel 4.5.3), bestätigte die Qualität unserer Messungen und Ergebnisse.

\section{Traumafragebogen}

Auf Grund des Fehlens eines standardisierten Fragebogens, der die Schweregrade der Missbrauchserfahrungen misst, war es erforderlich einen eigenen Traumafragebogen zu entwickeln. Dies geschah in enger Anlehnung an die in der Literatur vorgegebenen Definitionen (vgl. McGee 1995). In Kapitel 3.1.4 ist die Herleitung der Definitionen in Analogie zu McGee beschrieben.

Für eine Überprüfung der Aussagen des Traumafragebogens wurde in Tabelle 74 verglichen, inwieweit die Angaben aus den beiden Fragebögen EuropASI und Traumafragebogen bei den gleichen Patienten übereinstimmten. Dies war möglich, da sowohl im EuropASI als auch im Trauma-Fragebogen Angaben zu 
sexuellen, emotionalen und körperlichen Misshandlungen gemacht wurden, wenn auch mit jeweils eigenen Fragestellungen. Die folgende Tabelle zeigt das Ergebnis.

Tabelle 74: Übereinstimmung der Aussagen zu Missbrauchserfahrungen ( $n=83)$

\begin{tabular}{llcc}
\hline & & ja & nein \\
\hline Sexueller Missbrauch & EuropASI & $26(31 \%)$ & $57(69 \%)$ \\
\hline & Trauma-Frabo & $27(33)$ & $56(67 \%)$ \\
\hline Emotionaler Missbrauch & & & \\
\hline & EuropASI & $58(70 \%)$ & $25(30 \%)$ \\
\hline & Trauma-Frabo & $56(68 \%)$ & $27(32 \%)$ \\
\hline Körperlicher Missbrauch & EuropASI & $48(58 \%)$ & $35(42 \%)$ \\
\hline & Trauma-Frabo & $45(54 \%)$ & $38(46)$ \\
\hline
\end{tabular}

Am höchsten war die Übereinstimmung bei der Frage nach sexuellem Missbrauch. Hinsichtlich des körperlichen und emotionalen Missbrauchs gehen die Angaben in den beiden Fragebögen bei 2-3 Personen bzw. um etwa 3-4\% auseinander. Dies ist zu erklären mit der genaueren Fragestellung im Traumafragebogen, die eine bessere Unterscheidung des Vorliegens eines Missbrauchs aus Sicht der Patienten erlaubt. Prinzipiell wird aber durch dieses Ergebnis - der weitgehenden Übereinstimmung des EuropASIs und Traumafragebogens - deutlich, dass die Definitionen des Traumafragebogens nach McGee sinnvoll ausgewählt sind.

Ergänzend lässt sich festhalten, dass die Aussagen des Traumafragebogens lediglich die Analyse über die Auswirkungen des Schweregrad des Missbrauchs in Kapitel 3.2.8 und Kapitel 4.2 betreffen. Alle anderen Aussagen der Studie beruhen auf den validierten Erhebungen mit EuropASI und SCL 90-R und gelten somit unabhängig vom Traumafragebogen.

Erst kommende Untersuchungen mit dem hier eingeführten Traumafragebogen können die Objektivität, Reliabilität und Validität der Methode zur Unterscheidung 
des Schweregrads des Missbrauchs endgültig nachweisen. Als nächsten Schritt schlagen wir deshalb vor, dass eine weiterführende Arbeit an Hand des Traumafragebogens die Untersuchung einer Referenzgruppe vornehmen sollte. 


\section{Zusammenfassung}

Ziel dieser Arbeit war es, eine Aussage zu den Auswirkungen einer Missbrauchserfahrung in der Kindheit bei später drogenabhängigen PatientInnen zu treffen. Besonderer Fokus lag dabei auf der Frage, ob ein Missbrauch Auswirkungen auf die spätere Psychopathologie (Symptomentwicklung) hat und damit eventuell zu spezifischen Symptomkomplexen führt sowie mit einem schlechteren Verlauf der Suchterkrankung assoziiert ist. Dabei sollte der Einfluss der Missbrauchsart berücksichtigt werden. Ferner sollte beantwortet werden, ob die Schwere des Missbrauchs einen Einfluss auf die spätere Suchterkrankung bzw. die Symptombelastung der drogenabhängigen PatientInnen hat. Außerdem sollte untersucht werden, ob geschlechtsspezifische Unterschiede hinsichtlich Sucht und Symptombelastung zu finden sind.

\section{Einfluss einer Missbrauchserfahrung auf Problembereiche nach EuropASI}

Es war festzustellen, dass die PatientInnen, die eine Missbrauchserfahrung erlitten haben, sich nur in wenigen der betrachteten Problembereiche nach EuropASI signifikant von der Referenzgruppe ohne die Missbrauchserfahrung unterschieden: Hervorzuheben waren hauptsächlich psychische und körperliche Probleme. Die Auswirkungen der Traumatisierung in der Kindheit auf die Gesamtheit der betrachteten Problembereiche nach EuropASI waren als gering bis mittel einzustufen.

\section{Einfluss einer Missbrauchserfahrung auf den Verlauf der Suchterkrankung} Anhand des Problembereichs "Drogengebrauch" nach EuropASI sollte gemessen werden, ob ein Missbrauch einen schlechteren Verlauf der Suchterkrankung nach sich zieht. Hier wurde kein signifikanter Unterschied zwischen den Gruppen mit und ohne Missbrauch gefunden. Ein schlechterer Verlauf der Suchterkrankung auf Grund von Missbrauch konnte somit nicht bestätigt werden. Als Erklärung kann die hohe absolute Drogenbelastung (Composite Score von 0,7 ) beider Gruppen mit und ohne Missbrauchserfahrung herangezogen werden, 
die auf der Auswahl der Studienpopulation in Suchtstationen psychiatrischer Krankenhäuser beruht.

\section{Einfluss einer Missbrauchserfahrung auf die Entwicklung psychischer Probleme}

Bemerkenswert war die Analyse der psychischen Probleme nach SCL 90-R: Hier zeigte sich, dass die PatientInnen, die eine Missbrauchserfahrung erlitten haben, sich in einer Vielzahl von psychischen Symptomen signifikant von der Vergleichsgruppe ohne den jeweiligen Missbrauch abhoben. Bei fast allen Missbrauchsarten traten die Symptome Somatisierung, Unsicherheit im Sozialkontakt, Depressivität, Ängstlichkeit, phobische Angst und paranoides Denken signifikant auf. Auch die globalen Kennwerte GSI, PSDI und PST wurden überwiegend signifikant. Ein Missbrauch in der Kindheit war mit starken psychischen Problemen assoziiert, eine hohe Symptombelastung war mit unseren Ergebnissen nachzuweisen.

Insbesondere sexueller Missbrauch in der Kindheit war mit hohen, spezifischen psychischen Problemen verbunden. Ein angestellter Vergleich der psychischen Belastungswerte unserer suchtkranken Patienten mit sexueller Missbrauchserfahrung zu den Belastungswerten schizophrener Patienten bei Franke wies eine hohe Übereinstimmung auf. Die globalen Kennwerte, die eine übergeordnete Zusammenfassung der psychischen Symptombelastung geben, waren nahezu identisch. Unser Ergebnis zeigte klar, dass suchtkranke Patienten mit einer sexuellen Missbrauchserfahrung in der Kindheit die am schwersten belastete Patientengruppe war und sich deutlich von den anderen Missbrauchsgruppen bzw. der Gruppe ohne Missbrauch differenzierte.

Am zweitstärksten wirkte sich eine Vernachlässigung in der Kindheit auf die psychische Symptombelastung aus, gefolgt von familiärer Gewalt und emotionalem Missbrauch und zuletzt von körperlicher Misshandlung. 


\section{Geschlechtsspezifische Unterschiede}

Geschlechtsspezifische Unterschiede waren in unseren Ergebnissen nur in zwei Bereichen nachzuweisen. Lediglich „phobische Angst“ und „Unsicherheit im Sozialkontakt" fiel für Frauen zum Teil bei körperlichem Missbrauch, Vernachlässigung und emotionalem Missbrauch signifikant schlechter aus. Diese bei uns gefundene Symptombelastung deckt sich mit den in der Literatur beschriebenen Reaktionen bei Traumatisierungen durch Missbrauch. Eine weitere Differenzierung bezüglich der Schweregrade der Missbrauchsarten erbrachte keine zusätzlichen Unterschiede der Geschlechter hinsichtlich des Zustands und der psychischen Symptombelastung.

\section{Differenzierung des Missbrauchs nach dem Schweregrad}

Die Ergebnisse zeigten, dass eine Unterteilung in Schweregrade bei sexuellem Missbrauch und familiärer Gewalt in jedem Fall durchzuführen ist, da die jeweiligen Schweregrade deutlich mit einer signifikanten, differenzierten psychischen Symptombelastung einhergehen. Auch bei emotionalem Missbrauch und körperlicher Gewalt waren noch einige Kennwerte bei einer Unterteilung der Schweregrade signifikant. Hier kann ebenfalls eine Unterscheidung in die Schweregradkategorien vorgenommen werden. Lediglich bei Vernachlässigung traten keine Unterschiede bezüglich der Kategorisierung nach Schweregrade zu Tage. Die Schweregrade des Missbrauchs sind bei einer Gruppenaufteilung für die Therapie der Patienten zu beachten.

\section{Ansatz einer Gruppeneinteilung unter Berücksichtigung der Missbrauchsart, des Schweregrads und des Geschlechts für eine differenzierte Therapie}

Die Ergebnisse der Arbeit zeigten eindeutig, dass die verschiedenen Missbrauchsformen unter Berücksichtigung der Klassifizierung in Schweregrade mit signifikant unterschiedlichen psychischen Symptombelastungen assoziiert sind. Eine Einteilung in Gruppen, wie dies auch in der Literatur u.a. von Rounsaville (1982), Krausz und Müller-Thomsen (1994), Krausz und Freyberger 
(1997) gefordert wird, wurde ausgearbeitet. Die vorgenommene Einteilung berücksichtigt die signifikant gewordenen Kriterien jeder Gruppe und korreliert mit der Intensität der psychischen Symptombelastung:

- Sexueller Missbrauch mit Unterscheidung in leichten \& mittleren Schweregrad vs. schweren Missbrauch

- Vernachlässigung ohne Unterscheidung des Schweregrades

- Familiäre Gewalt mit Unterscheidung in leichten \& mittleren Schweregrad vs. schweren Missbrauch

- Emotionaler Missbrauch mit Unterscheidung in leichten \& mittleren Schweregrad vs. schweren Missbrauch

- Körperliche Misshandlung ohne Unterscheidung des Schweregrades

- Kein Missbrauch

Eine Therapie für Suchtpatienten sollte aus unserer Sicht die höheren psychischen Probleme der jeweiligen Missbrauchsgruppe berücksichtigen. Hierzu wird auch empfohlen, die Ursachen für die erhöhte psychische Symptombelastung - sprich die jeweilige Missbrauchserfahrung - heranzuziehen und in die Therapie zu integrieren. Dies verspricht gezieltere und bessere Therapiechancen für die drogenkranken PatientInnen, die eine Missbrauchserfahrung in der Kindheit erlitten haben.

\section{Schlussfolgerung}

Die Arbeit kann unsere anfängliche Hypothese belegen, dass Suchtpatienten mit Missbrauchserfahrung in der Kindheit einer vielfach höheren psychischen Symptombelastung ausgesetzt sind als Suchtpatienten, die keine Missbrauchserfahrung erlitten haben. Allerdings fand sich kein Beleg für die Annahme, dass die in ihrer Kindheit missbrauchten bzw. misshandelten Patienten mit einer späteren Drogenabhängigkeit sich durch einen erhöhten Konsum und spezifisch mehr Probleme in dem durch den EuropASI erfassten Problembereich "Drogen“ auszeichnen. Dies führten wir auf die Auswahl der Studienpopulation zurück. Ferner hatten verschiedene Missbrauchsarten 
unterschiedliche Auswirkungen auf die spätere Symptombelastung. Es wird deutlich, wie wichtig eine Einteilung der Drogenpatienten in Gruppen ist, um die jeweiligen Missbrauchserfahrungen in der Kindheit in die Therapie zu integrieren. Damit kann eine differenzierte Therapie unter Berücksichtigung der Ursache der Symptombelastung ermöglicht und die Therapiechance für die Drogenpatienten verbessert werden. 


\section{Literaturverzeichnis}

Abraham, K. (1908): Die psychologischen Beziehungen zwischen Sexualität und Alkoholismus. Sexualwiss, 8, 449-458.

Amann, G. und Wipplinger, R. (1997): Zur Bedeutung der Bezeichnungen und Definitionen von sexuellem Mißbrauch. In: Amann, G. und Wipplinger, R. (Hrsg.): Sexueller Mißbrauch: Überblick zu Forschung, Beratung und Therapie. DgvtVerlag, Tübingen, 13-39.

Arenz-Greiving, I. (1994): Suchtkranke Mütter rund ihre Kinder. In: Arenz-Greiving, I. \& Dilger, H. (Hrsg.). Elternsüchte-Kindernöte, Berichte aus der Praxis, Freiburg im Breisgau: Lambertus, 15-49.

B. Gsellhofer, H. Küfner, M. Vogt und D. Weiler (1994): European Addiction Severity Index EuropASI. Manual für Training und Durchführung. Hohengehren: Schneider (nach der 5. Auflage der amerikanische Version von McLellan und der Europäischen Version des ASI).

Bagley, C. (1995): Child sexual abuse an mental health in adolescents and adults. British and Canadian perspectives. Aldershot: Avebury.

Bagley, C. and King, K. (1990): Child sexual abuse: The search for healing, London: Tavistock/Routledge.

Bange, D. (1992): Die dunkle Seite der Kindheit. Sexueller Mißbrauch an Mädchen und Jungen - Hintergründe - Folgen. Köln: Volksblattverlag.

Bange, D. and Deegener, G. (1996): Sexueller Missbrauch an Kindern: Ausmaß, Hintergründe, Folgen, Weinheim: Beltz, PVU.

Barnett, D., Manley, J.T., Cicchetti, D. (1993): Defining child maltreatment; the interface between polica and research. In: Child abuse, child development, and social policy, Cicchetti, D. Toth, S.L. (eds.), Norwood / NJ: Ablex, 7-74.

Baydar, N. \& Brooks-Gunn, J. (1991): Effects of maternal employment and childcare arrangements on preschoolers' cognitive and behavioural outcomes: 
evidence from the children of the National Longitudinal Survey of Youth. Develop Psychol, 27, 932-945.

Beitchman, J.H., Zucker, K.J., Hood, J.E., daCosta, G.A. \& Akman, D. (1991): A review of the short-term effects of child sexual abuse. Child Abuse \& Neglect, 15, 537-556.

Beitchman, J.H., Zucker, K.J., Hood, J.E., DaCosta, G.A., Akman, D. and Cassavia, E.A. (1992): A review of the long term effects of child sexual abuse, Child Abuse Negl, 16, 101-118.

Bensley, L.S., Spieker, S.J., Eenwyk, J., Schoder, J. (1999): Self-Reported Abuse History and Adolescent Problem Behaviour. II. Alcohol and Drug Use, Elsevier Science, Journal of Adolescent Health, 1999, 24, 173-180.

Besharov, D.J. (1993): Overreporting and underreporting are twin problems. In: Gelles, R.J. and

Beutel, M. (1999): Sucht und sexueller Missbrauch: Eine empirische Untersuchung, Psychotherapeut, 44: 313-319.

Bilfuco, A., Brown, G.W. \& Adler, Z. (1991): Early sexual abuse and clinical depression in adult life, British Journal of Psychiatry, 159: 115-122.

Bortz, J.; Döring, N. (1995). Forschungsmethoden und Evaluation, 2. Auflage,

Briere, J. \& Runtz, M. (1988): Symptomatology associated with childhood sexual victimization in a nonclinical adult sample. Child Abuse \& Neglect, 12: 51-59.

Briere, J. \& Zaidi, L.Y. (1989): Sexual abuse histories and sequalae in female psychiatric emergency room patients. Am J Psychiat, 146: 1602-1606.

Briere, J. (1992): Child abuse trauma: Theory and treatment of the lasting effects. Newbury Park, CA: Sage.

Briere, J. and Runtz, M. (1990): Different adult symptomatology associated with three types of child abuse histories, Child Abuse Negl, Vol. 14, 357 - 364

Brockhaus, U. und Kolshorn, U. (1993): Sexuelle Gewalt gegen Mädchen und Jungen. Mythen, Fakten, Theorien. Frankfurt: Campus. 
Brown , G.R. and Anderson, B. (1991): Psychiatric morbidity in adult inpatients with childhood histories of sexual and physical abuse, Am J Psychiat, 148, 55 61.

Browne, A. und Finkelhor, D. (1986):Impact of child sexual abuse; A review of the Research, Psychological Bulletin, 99, 6-77

Bryer J.B., Nelson B.A., Miller J.B. Krol P.A. (1987): Childhood sexual and physical abuse as factors in adult psychiatric illness, AM J Psychiat, 144, 14261430

Bulic C., Sullivan P., Rorty M. (1989): Childhood sexual abuse in women with bulimia, J Clin Psychiat, 50, 460-466

Bundesministerium für Familie, Senioren, Frauen und Jugend (2000): Kindesmisshandlung. Erkennen und Helfen, Kinderschutzzentrum Berlin (Hrsg.), Berlin.

Caffaro-Rouget, A., Lang, R.A. and van Santen, V. (1989): The impact of child sexual abuse. Annals of Sex Research, 2, 29-47.

Cavaiola, A.A. and Schiff, M. (1988): Behavioral sequelae of physical and/or sexual abuse in adolescents. Child Abuse Negl, 12, 181 - 188

Chu, J.A. \& Dill, D.L. (1990): Dissociative symptoms in relation to childhood physical and sexual abuse. Am J Psychiat, 147: 887-892.

Cohen, F.S. and Densen-Gerber, J. (1982): A study of the relationship between child abuse and drug addiction in 178 patients: preliminary results, Child Abuse Negl, 6, 383 - 387.

Cole, P.M. \& Putnam, F.W. (1992): Effects of incest on self and social functioning: A developmental psychopathology perspective. Journal of Consulting and Clinical Psychology, 60, 174-184.

Coleman, M.M., Alder, M.E. and Prihoda, T.J. (1995): Regional comparisons of child abuse and related variables in the United States, Am J Forensic Med Pathol, 16(4), 314-319 
considerations, criminal justice and behaviour, 19, 194 - 216

Conte, J. \& Schuermann, J. (1987): The effect of sexual abuse on children: A multidimensional view. Journal of Interpersonal Violence, 2, 380-390.

Conte, J.R. (1993): Sexual abuse of children. In: Hampton, R.L., Gullota, T.P., Adams, G.R., Potter, E.H. and Weissberg, R.P. (Eds., Family violence: Prevention and treatment, 56-85, Newbury Park: Sage.

Coons P.M. (1986): Child abuse and multiple personality disorders: review of the literature and suggestions for treatment. Child Abuse \& Neglect, 15, 293-301

Damlouji N.F., Ferguson J.M. (1985): Three cases of postramatic anorexia nervosa, AM J Psychiat, 142, 262-263

De Bellis, M. (2002) : Developmental traumatology : a contributory mechanism for alcohol and substance use disorder, Elsevier Science, Psychoneuroendocrinolgy 27, 155 - 170.

Derogatis, L. R. \& Savitz, K. L. (1999). The SCL-90-R, Brief Symptom Inventory, and matching clinical rating scales. In M. E. Maruish (Ed.), The use of psychological testing for treatment planning and outcome assessment (2nd ed.), 679-724.

Derogatis,-L. R. \& Cleary,-P. A. (1977). Factorial invariance across gender for the primary symptom dimensions of the SCL-90. British-Journal-of-Social-andClinical-Psychology 16: 347-356

Dilling, H., Mombour, W., Schmidt, M.H. (Hrsg.) (1993): Internationale Klassifikation psychischer Störungen, ICD-10, Kapitel V (F). Klinischdiagnostische Leitlinien, 2.Aufl. Bern: Huber.

Dilling, H., Mombour, W., Schmidt, M.H., Schulte-Markwort, E. (Hrsg.) (1994): Internationale Klassifikation psychischer Störungen, ICD-10, Kapitel V (F). Klinisch-diagnostische Leitlinien, 2.Aufl. Bern: Huber.

Dornes, M. (1997): Vernachlässigung und Misshandlung aus der Sicht der bindungstheorie. In: Amann, G. und Wipplinger, R. (Hrsg.): Sexueller Mißbrauch: 
Überblick zu Forschung, Beratung und Therapie. Dgvt-Verlag, Tübingen, S. 64 79 .

Draijer, N. (1990): Die Rolle von sexuellem Mißbrauch und körperlicher Mißhandlung in der Ätiologie psychischer Störungen bei Frauen. System Familie, $3,59-73$

Dührssen, A. (1984): Risikofaktoren für die neurotische Kindheitsentwicklung. Ein Beitrag zur psychoanalytischen Geneseforschung. Z Psycho-Som Med, 30, 1842.

Egle U., Hoffmann S., Joraschky P. (1997): Sexueller Missbrauch, Misshandlung, Vernachlässigung: Erkennung und Behandlung psychischer und psychosomatischer Folgen früher Traumatisierung, Stuttgart Schattauer, S.3

Egle, U., Hoffmann, S.O. und Steffens, M. (1997): Pathogene und protektive Entwicklungsfaktoren in der Kindheit und Jugend. In: Egle, U.T., Hoffmann, S.V. und Joraschky, P. (Hrsg.): Sexueller Mißbrauch, Mißhandlung, Vernachlässigung. Schattauer Verlag, Stuttgart, 3 - 21

Elliger, T.J. und Schrötensack, K. (1991): Sexueller Mißbrauch von Kindern eine kritische Bestandsaufnahme. In: Nissen G. (Hrsg.): Psychogene Psychosyndrome und ihre Therapie im Kindes- und Jugendalter. Bern, Huber, $143-154$

Endres, J. und Scholz, O.B. (1994): Sexueller Kindesmissbrauch aus psychologischer Sicht, NStZ, Heft 10, 466-473

Engfer, A. (1991): Prospective identification of violent mother-child relationships. Child outcome at 6.3 years. In: Victims and criminal justice. Vol III. Special victimological issues. Particular groups of victims. Kaiser, G., Kury, H. , Albrecht, H.J.(eds). Freiburg: Max-Planck-Institut für Ausländisches und Internationales Strafrecht, 415-458.

Engfer, A. (1992): Kindesmisshandlung und sexueller Missbrauch. Zeitschrift für Pädagogische Psychologie, 6: 165-174.

Erickson, M.F., Egeland, B., Pianta, R. (1989): The effects of maltreatment on the 
development of young children. In: Child maltreatment. Theory and research on the causes and consequences of child abuse and neglect. Cicchetti D., Carlson, V. (eds.), Cambridge: Cambridge University Press, 647-684.

Ernst, C (1997): Zu den Problemen der epidemiologischen Erforschung des sexuellen Missbrauchs. In: Amann, G. und Wipplinger, R. (Hrsg.): Sexueller Mißbrauch: Überblick zu Forschung, Beratung und Therapie. Dgvt-Verlag, Tübingen

Esser, G. (1994): Ablehnung und Vernachlässigung im Säuglingsalter. In: Die vergessenen Kinder. Vernachlässigung und Armut in Deutschland. Kürner, P., Nafroth, R. (Hrsg.), Köln: PapyRossa, 72-80.

Faller, K.C. (1989): Characteristics of a clinical sample of sexually abused children: How boy an girl victims differ, Child Abuse Negl, 13, $101-110$

Fegert, J. (1992): Diagnostik und klinisches Vorgehen bei Verdacht auf sexuellen Missbrauch bei Mädchen und Jungen. In: Walter, J. (Hrsg.), Sexueller Missbrauch im Kindesalter (2. Aufl.), Heidelberg: HVA/Edition Schindele, 68-101. Finkelhor, D. \& Baron, L. (1986): High risk children. In: Finkelhor, D. (ed): A sourcebook on child sexual abuse, Beverly Hills: Sage, 60-88.

Finkelhor, D. (1984): Child sexual abuse: New theory and research, New York: Free Press

Finkelhor, D. (1997): Zur internationalen Epidemiologie von sexuellem Mißbrauch an Kindern. In: Amann, G. und Wipplinger, R. (Hrsg.): Sexueller Mißbrauch: Überblick zu Forschung, Beratung und Therapie. Dgvt-Verlag, Tübingen, S. 72 89

Finkelhor, D., Browne, A. (1985): The traumatic impact of child sexual abuse: A conceptualization, American Journal of Orthopsychiatry 55, 530-541

Fischer,G. (1998): Lehrbuch der Psychotraumatologie, München (Ernst Reinhart), 255

Fischer,G. (1998): Lehrbuch der Psychotraumatologie, München (Ernst 
Reinhart), 248ff

Flügel, A. \& Merfert-Diete, C. (1993): Frauenspezifische Therapie, In: Deutsche Hauptstelle gegen die Suchtgefahren (Hrsg.): Jahrbuch Sucht 1996, Geestacht, Neuland.

Franke, G. H. (1992). Eine weitere Überprüfung der Symptom-Check-Liste (SCL90-R) als Forschungsinstrument. Diagnostica, 38, 160-167.

Franke, G.H. \& Stäcker, K.-H. (1995). Reliabilität und Validität der SymptomChecklist (SCL-90-R,Derogatis, 1986) bei Standardreihenfolge versus inhomogener Blockbildung. Diagnostica 41: 349-373

Franke, G.H. (1995). Die Symptom-Checkliste von Derogatis - Deutsche Version - Manual. Göttingen: Beltz Test GmbH

Friedmann R., Hurt S., Clarkin J., Corn R., Aronoff M. (1982): Sexual histories and premenstrual affective syndrome in psychiatric inpatients, AM J Psychiat, 139, 1484-1486

Fromuth, M.E. (1986): The relationship of childhood sexual abuse with later psychological and sexual adjustment in a sample of college women. Child Abuse \& Neglect, 10: 5-15.

Fürstenberg, F. \& Teitler, J.O. (1994): Reconsidering the effects of marital disruption. What happens to children of divorce in early adulthood? J Fam Issues, 15, 173-190.

Gaenslen-Jordan, C., Appelt, H. und Osterroht, A.. (1990): Sexueller Missbrauch von Mädchen in der Familie - Ergebnisse einer Auswertung psychologischer Glaubwürdigkeitsgutachten. Psychotherapie, Psychosomatik und medizinische Psychologie, 40, 241 - 247.

Garmezy, N. (1985): Stress-resistant children: the search for protective factors. In: Stevenson, J.F. (ed). Resent research in developmental psychopathology. Oxford: Pergamon Press, 213-233.

Gast, U. (1995): Einfluss der Psychoanalyse auf die Erforschung und 
Behandlung schwerer sexueller Traumata. In: Gesellschaftliche Umbrüche individuelle Antworten. Aufgaben der psychosomatischen Medizin. Senf, w., Heuft, G. (Hrsg.), Frankfurt: VAS.

Geier, B. (1990): Genese, Dynamik und Therapie bei sexuellem Missbrauch. In: Arenz-Greiving, I. (Hrsg.), Sucht - Gewalt - Sexualität: Opfer und Täter in der Therapie, Freiburg im Breigau: Lambertus, 37-48.

Gold E.R. (1986): Long-term effects of sexual victimization in childhood: an attributional approach, J Consult Clin Psychol, 54, 173-189

Goodwin, J., McCarty, T. and DiVasto, P. (1982): Physical and sexual abuse of the children of adult victims. In: J. Goodwin (a.a.O.).

Goodwin, J., Simms, M. and Bergman, R.(1979): Hysterical seizures: A sequel to incest, Am J Orthopsychiatry 49: $698-703$.

Greenwald E., Leitenberg H., Cado S., Tarran M., (1990): Childhood sexual abuse: long term effects on a psychological and sexual functioning in a nonclinical and nonstudent sample of adult woman, Child Abuse \& Neglect, 14, 503-513

Gunderson, J.G. \& Sabo, A.N. (1993): The phenomenological and conceptual interface between borderline personality disorder and PTSD. Am J Psychiat, 150: 19-27.

Harter, S., Alexander, P.C. \& Neimeyer, R.A. (1988): Long-term effects of incestuous child abuse in child abuse in college women: social adjustment, social cognition and family characteristics. J Consult Clin Psychol, 56: 5-8.

Hedrich, D. (1989): Drogenabhängige Frauen und Männer, In: Kindermann, W. (Hrsg.): Drogenabhängig: Lebenswelten zwischen Szene, Justiz, Therapie und Drogenfreiheit, Freiburg, Lambertus.

Heigl-Evers, A. \& Schultze-Dierbach, E. (1983): Überlegungen zur Indikation von Einzel- und Gruppentherapie bei Suchtkranken, insbesondere Alkoholkranken. Sozialtherapie in der Praxis II. Kassel. 
Herman J.L. (1994): Die Narben der Gewalt. Traumatische Erfahrungen verstehen und überwinden, München: Kindler.

Hermann, J.L., Perry, J.C., van der Kolk, B.A. (1989): Childhood trauma in borderline personality disorder. Am J Psychiat, 146: 490-495.

Hirsch, M. (1987): Realer Inzest: Psychodynamik des sexuellen Mißbrauchs in der Familie, Berlin, Heidelberg, New York, London, Paris, Tokyo; Springer.

Ireland, T. and Widom, C.S. (1994): Childhood victimization and risk for alcohol and drug arrest, Int J Addiction, 29, 235 - 274.

Karren-Derber, U. (1989): Sexueller Missbrauch von Kindern und Jugendlichen und Suchtmittelmissbrauch. Praxis der Klinischen Verhaltensmedizin und Rehabilitation, 4: 174-178.

Kavemann, B. \& Lohstöter, I. (1986): Väter als Täter: Sexuelle Gewalt gegen Mädchen. Hamburg, Rowohlt.

Kempe, C.H. (1968): Incest and other forms of sexual abuse. In: Kempe, C.H. \& Helfer, R.E. (eds.): The battered child. The University of Chicago Press, Chicago \& London

Kendall-Tackett, K.A., Williams, L.M. and Finkelhor, D. (1993): Impact of sexual abuse on children: a review and synthesis of recent empirical studies, Psychol Bull, 113, $164-180$.

Kendler et al. (2000) Arch Gen Psychatry57: 953 (siehe auch Präsentation Martin)

Kernberg, O.F. (1978): Borderline-Störung und Pathologischer Narzissmus. Frankfurt: Suhrkamp.

Kernberg, O.F. (1989): Schwere Persönlichkeitsstörungen: Theorie, Diagnose, Behandlungsszenarien. Klett-Cotta Stuttgart.

Kernberg, O.F., Selzer, M.A. Koenigsberg, H.W., Carr, A.C., Appelbaum, A.H. (1993): Psychodynamische Therapie bei Borderline-Patienten. Bern: Huber. 
Kinsey, A.A. (1953): Sexual behaviour in the human female. Philadelphia: Saunders \& Co

Kinzl, J. and Biebl, W. (1991): Sexual abuse of girls: aspect of the genesis of mental disorders and therapeutic implications, Acta Psychiatr Scand , 83, 427 431

Koeter \& Hartgers (1997): Preliminary procedure for the computation of the EuropASI composite scores. The Amsterdam Institute for Addiction Research.

Kolko, D.J. (1992): Characteristics of child victims of physical violence: Research findings and clinical implications. J Interpersonal Violence, 7, 244-276

Krausz, M. und Müller-Thomsen, T.(Hrsg.) (1994): Komorbidität. Therapie von psychischen Störungen und Sucht - Konzepte für Diagnostik, Behandlung and Rehabilitation, Heidelberg: Lambertus

Krausz, M., Freyberger H.J. (1997): Suchterkrankungen. In Egle, U. T., Hoffmann, S. V., Joraschky, P. (Hrsg): Sexueller Missbrauch, Misshandlung, Vernachlässigung. Schattauer Verlag, Stuttgart $284-293$

Kreyssig, U. (1997): Der Zusammenhang von sexuellem Mißbrauch und Sucht. In: Amann, G und Wipplinger, R. (Hrsg.): Sexueller Mißbrauch: Überblick zu Forschung, Beratung und Therapie. Dgvt-Verlag, Tübingen

Ladewig, D. \& Graw, P. (1985): Entwicklungschancen Drogenabhängiger, Weinheim, Beltz.

Lieberz, K. (1988): Was schützt vor Neurose? Ergebnisse einer Vergleichsuntersuchung an hochrisikobelasteten Neurotikern und Gesunden. Z Psychosom Med Psychoanal, 34, 338-350.

Lindberg F., Distad L. (1985): Post-traumatic stress disorders in women who experienced childhood incest. Child Abuse \& Neglect, 9, 329-334.

Lösel, F., Bliesener, T., Köferl, P. (1989): On the concepts of „invulnerability“: evaluation and first results of the Bielefeld Project. In: Children at risk: assessment, longitudinal research, and intervention. Brambring, M., Lösel, F., 
Skowronek, H. (eds), Berlin, New York: DeGruyter.

MacMillan, H.L., Flemming, J.E., Trocmé, N., Boyle, M.H., Wong, M., Racine, Y.A., Beardslee, W.R., Oxford, D.R. (1997): Prevalence of child physical and sexual abuse in the community; results form the Ontario Health Supplement, Journal of the American Medical Association, 278, S. 131-135

Malinowsky-Rummel, R. \& Hansen, D.J. (1993): Long-term consequences of childhood physical abuse. Psychol Bull, 114: 68-79.

Marcenko, M.O., Kemp, S.P., Larson, N.C. (2000): Childhood experiences of abuse, later substance use, and parenting outcomes among low-income mothers, Am J Orthopsychiatry, 70(3): 316-326.

Martinius, J. (1989): Persönlichkeitsentwicklung misshandelter Kinder. In: Retzlaff, I. (Hrsg.): Gewalt gegen Kinder, Jungjohann verlag, UIm.

Marwitz, G., Hörnle, R. und Luber, E.M. (1990): Prostitution als Bewältigungsform in der Kindheit erlittenen sexuellen Mißbrauchs mit seinen Folgen, Öff. Gesundh.-Wes., 52, 658 - 660

McGee, R.A. \& Wolfe, D.A. (1991): Psychological Maltreatment: Toward an operational definition, Development and Psychopathology, 3, 3-18.

McGee, R.A., Wolfe, D.A., Yuen, S.A., Wilson, S.K., Carnochan, J. (1995): The measeurement of maltreatment: A comparison of approaches, Child Abuse \& Neglect, Vol. 19, No. 2, Seite 233-249

McLellan, A. T., Kushner, H., Metzger, D., Peters, R., Smith, I., Grissom, G., Pettinati, H., \& Argeriou, M. (1992). The fifth edition of the Addiction Severity Index. Journal of Substance Abuse Treatment, 9, 199-213

Moggi, F. ( 1991): Sexuelle Kindesmisshandlung: Definition, Prävalenz und Folgen. Zeitschrift für Klinische Psychologie, Psychopathologie und Psychotherapie, 39, 323-335.

Moggi, F. (1997): Sexuelle Kindesmisshandlung: Traumatisierungsmerkmale, typische Folgen und ihre Ätiologie. In: Amann, G. und Wipplinger, R. (Hrsg.): 
Sexueller Mißbrauch: Überblick zu Forschung, Beratung und Therapie. DgvtVerlag, Tübingen, 187-200.

Moncrieff, J., Drummond, D.C., Candy, B., Checinski, K. and Farmer, R. (1996): Sexual abuse in people with alcohol Problems, Brit J Psychiat, 169, 335 - 360

Morrison J. (1989): Childhood sexual histories of women with somatization disorder, AM J Psychiat, 146, 239-241.

Mullen, P.E., Martin, J.L., Anderson, J.C. Romans, S.E. \& Herbison, G.P. (1996): The longterm impact of the physical, emotional and sexual abuse of children: $A$ community study. Child Abuse and Neglect, 20: 7-22.

Mullen, P.E., Martin, J.L., Anderson, J.C., Romans, S.E. and Herbison, G.P. (1993): Childhood sexual abuse and mental health in adult life, Brit J Psychiat, $163,721-732$

Muram, D. (1993): Child sexual abuse, Curr Opin Opstet Gynecol; 5(6), 784 - 790 Murphy , S.M. and Peters, F.S.D. (1992): Profiling child sexual abusers: psychological

Nash, M.R., Hulsey, T.L., Sexton, M.C., Harralson, T.L. \& Lambert, W. (1993): Long-term sequelae of childhood sexual abuse: Perceived family environment, psychopathology, and dissociation. Journal of Consulting and Clinical Psychology, 61, 276-283.

Ogata S., Silk K.R., Goodrich S., Lohr N.E., Westen D., Hill E.M. (1990): Childhood sexual and physical abuse in adult patients with borderline personality disorder, AM J Psychiat, 147, 1008-1013.

Peters, S.D. (1988): Child sexual abuse and later psychological problems. In: Wyatt, G. \& Powell, G. (eds): Lasting effects of child sexual abuse, Newbury Park, California: Sage, 101-117.

Pynoos, R.S., Nader, K. (1993): Issues in the treatment of posttraumatic stress in children and adolescents, In: Wilson ,J.P., Raphael, B. (1993), 535-550

Rado, S. (1926): Die psychischen Wirkungen der Rauschgifte. Psyche1975, 
29:360-366.

Rado, S. (1934): Psychoanalyse oder Pharmakotherapie? Int Z Psychoanal , 20: 16-32.

Raupp, U. \& Eggers, C. (1993): Sexueller Missbrauch von Kindern: Eine regionale Studie über Prävalenz und Charakteristik, Monatsschrift Kinderheilkunde, 141: 316-322.

Remschmidt, H. (1986): Körperliche Kindesmisshandlung, Münchner Medizinische Wochenschrift, 128(4), 33-36.

Richter-Appelt, H. (1994): Sexuelle Traumatisierung und körperliche Misshandlung. Eine Befragung von Studentinnen und Studenten. In: Rutschky, K. \& Wolff, R. (Hrsg.): Handbuch sexueller Missbrauch (S. 116-142), Hamburg: Klein.

Richter-Appelt, H. (1997): Differentielle Folgen von sexuellem Mißbrauch und körperlicher Mißhandlung. In: Amann, G. und Wipplinger, R. (Hrsg.): Sexueller Mißbrauch: Überblick zu Forschung, Beratung und Therapie. Dgvt-Verlag, Tübingen, S. $201-217$

Richter-Appelt, H., Kolb, J., Becker, C. \& Kawski, S. (1995): Körperliche Misshandlung und sexuelle Traumatisierung in der Kindheit aus der Sicht junger Erwachsener (Forschungsbericht für die Deutsche Forschungsgemeinschaft). Hamburg: Universitätskrankenhaus Eppendorf, Abteilung für Sexualforschung.

Romans, S.E., Martin, J.L., Anderson, J.C., O'Shea, M.L., Mullen, P.E. (1995): Factors that mediate between child sexual abuse and adult psychological outcome. Psychol Med, 25: 127-142.

Rose, S. M., Peabody, C.G. and Stratigeas, B. (1991): Responding to hidden abuse: A role for social work in reforming mental health system, Social Work, 35 , $408-413$.

Rost, W.D. (1987): Alkoholismus und Psychoanalyse. Stuttgart: Klett-Cotta. 
Rounsaville, B.J., Weissman, M.M., Wilber, C.H. and Kleber, H.D. (1982): Pathways to opiate addiction: An evaluation of differing antecedents. Brit $\mathrm{J}$ Psychiat, 141, $437-446$.

Rush, F. (1985): Das bestgehütete Geheimnis. Sexueller Kindermißbrauch, Berlin: Orlanda

Russell, D.E.H. (1983): The incidence and prevalence of intrafamilial and extrafamilial sexual abuse of female children. Child Abuse Negl, 7, 133 - 146

Russell, D.E.H. (1986): The secret trauma: incest in the lives of girls and women, New York, Basic Books.

Rutter, M. \& Quinton, D. (1984): Long term follow-up of women institutionalized in childhood: factors promoting good functioning in adult life. Brit $\mathrm{J}$ Develop Psychol, 18, 225-234.

Rutter, M. (1986): The developmental psychopathology of depression: issues and perspectives. In: Rutter, M., Izard, C., Read, P. (eds). Depression in young people. Developmental and clinical perspectives. New York: Guilford Press, 3-30. Sachsse, U. (1995): Die Psychodynamik der Borderlinestörung als Traumafolge. Ein Entwurf. Forum Psychoanal, 11: 50-61.

Salter, A.C. (1988): Treating child sex offender: A practical guide. London, Sage.

Saunders, B.E., Villeponteaux, L.A., Lipovsky, L.A. and Kilpatrick, D.G. (1992): Child sexual assault as a risk factor for mental disorders among women: $A$ communitiy survey., J Interpersonal Violence, 7, 189 - 204

Saunders, E.A. \& Arnold, F.A. (1993): A critique of conceptual and treatment approaches to borderline psychopathology. Psychiat, 56: 188-203.

Schäfer, M., Schnack, B., Soyka, M. (2000): Sexueller und körperlicher Missbrauch während früher Kindheit oder Adoleszenz bei späterer Drogenabhängigkeit, PPmP Psychotherapie Psychosomatik Medizinische Psychologie, Georg Thieme Verlag Stuttgart, 50(2), 38-50 
Schei, B. (1990): Prevalence of sexual abuse history in a random sample of norwegian women, Scand J Soc Med, 1, 63 - 68

Schepank, H. (1987): Psychogene Erkrankungen in der Stadtbevölkerung. Eine epidemiologische-tiefenpsychologische Untersuchung in Mannheim. Berlin: Springer.

Schetky, D.H. (1990): A review of the literature on the long-term-effects of childhood sex abuse. In: Kluft, R.P. (ed). Incest related syndromes of adult psychopathology. Washington/DC; American Psychiatric Press, 35-55.

Scheurich, A, Mueller, MJ, Wetzel, H, Anghelesu, I, Klawe, C, Ruppe, A, Lorch, B, Himmerich, H, Heidenreich, M, Schmid, G, Hautzinger, M, Szegedi, A (2000): Reliability and validity of the German version of the European Addiction Severity Index (EuropASI), Stud Alconol, 61(6): 916-9

Scott, K.D. (1992): Childhood sexual abuse: Impact on a community's mental health status, Child Abuse Negl, 16, 285 - 295

Sedney, M.A. \& Brooks, B. (1984): Factors associated with a history of childhood sexual experience in a nonclinical female population. J Am Acad Child Psychiat, 23: $215-218$.

Seifer, R., Dameroff, A.J., Baldwin, C.P., Baldwin, A..(1992): Child and family factors that ameliorate risk between 4 and 13 years of age. J Am Acad Child Adolesc Psychiat, 31, 893-903.

Sgroi, S.M., Canfield Blick, L.., Sarnacki Porter, F. (1982): A conceptual framework for child sexual abuse. In: Sgroi, S.M. (eds.), Handbook of clinical intervention in child sexual abuse, Lexington, MA: Lexington Books, 9-37.

Shengold, L. (1995): Soul Murder. The effects of childhood abuse and deprivation. New Haven, London: Yale University Press.

Simpson, T.L. \& Miller, W.R. (2002): Concomitance between childhood sexual and physical abuse and substance use problems, Clinical Psychology Review, 22 (1), 27-77. 
Singer, M.I., Petchers, M.K. and Hussey, D. (1989): The relationship between sexual abuse and substance abuse among psychiatrically hospitalized adolescents, Child Abuse Negl, 13, 319 - 325

Sloan G., Leichner P. (1986): Is there a relationship between sexual abuse or incest and eating disorder? Canad J Psychiat, 31, 656-660.

Soyka, M. (1997): Alkoholismus - eine Krankheit und ihre Therapie, Wissenschaftliche Verlagsgesellschaft, Stuttgart.

Soyka, M. (1998): Drogen- und Medikamentenabhängigkeit. Wissenschaftliche Verlagsgesellschaft, Stuttgart.

Springer- Verlag, Berlin, Heidelberg, New York, 216- 234.

Stein, J.A., Golding, J.W., Siegel, J.M., Burnam, M.A. and Sorenson, S.B. (1988): Long-term psychological sequale of child sexual abuse: The Los Angeles epidemiologic catchment area study. Lasting Effects of Children Sexual Abuse (pp. 135 - 145). Newbury Park, CA: Sage.

Sullivan, P.F., Bulik, C.M. and Carter, F.A. (1995): The significance of a history of childhood sexual abuse in bulimia nervosa, Brit J Psychiat, 167, 679 - 682

Swett, C. and Halpert, M. (1993): Reported history of physical and sexual abuse in relation to dissociation and other symptomatology in women psychiatric inpatients, J Interpersonal Violence, 8, 545- 555.

Terr, L. (1995): Schreckliches Vergessen, heilsames Erinnern. Traumatische Erfahrungen drängen ans Licht. München (Kindler).

Teusch, R. (2001): Substance abuse as a symptom of childhood sexual abuse, Psychiatr Serv 2001, 52(11): 1530-1532.

Thornberry, T.P., Ireland, T.O., Smith, A.C. (2001): "The importance of timing: The varying impact of childhood and adolescent maltreatment on multiple problem outcomes", Cambridge University Press, Development and Psychopathology, 13, p. 957-979. 
Triffleman EG, Marmar CR, Delucchi KL, Ronfeldt H. (1995): Childhood trauma and posttraumatic stress disorder in substance abuse inpatients, J Nerv Ment Dis, 183

(3), $172-6$

Downs, W.R., Harrison, L. (1998): Childhood maltreatment and the risk of substance problems in later life, Blackwell Science, Health and Social Care in the Community, 6(1), 35-46.

Ulrich, M. (1988): Risiko und Schutzfaktoren in der Entwicklung von Kindern und Jugendlichen, Z Entwicklungspsychol Pädagog Psychol, 20 , 146 - 166

Van der Kolk, B.A. \& Fisler, R.E. (1994): Childhood abuse and neglect and loss of self-regulation. Bull Menninger Clin, 58: 417-421.

van der Kolk, B.A., Greenberg, M.S., Orr, S.P., Pitman, R.K. (1989): Endogenous opioids, stress induced analgesia, and Posttraumatic Stress Disorder, Psychopharmacol Bull, 25: 417-421

van der Kolk, B.A., Perry, J.C., Herman, J.L. (1991): Childhood origins of selfdestructive behavior. Am J Psychiat, 148: 1665-1671.

Vize, C.M. and Cooper, P.J. (1995): Sexual abuse in patients with eating disorder, patients with depression and normal controls. A comperative study, Brit J Psychiat, 167, 80 - 85

Wallen, J. (1992): A comparison of male and female clients in substance abuse treatment. Journal of substance abuse treatment, 9, 243-248.

Walper, S. \& Silbereisen, R.K. (1986): Individuelle und familiäre Auswirkungen ökonomischer Einbußen. In: Bericht über den 35. Kongress der Deutschen Gesellschaft für Psychologie in Heidelberg. Bd. 1. Göttingen: Hofgrefe, 298.

Walper, S. \& Silbereisen, R.K. (1987): Familiäre Konsequenzen ökonomischer Einbußen und ihre Auswirkungen auf die Bereitschaft zu normverletzendem Verhalten bei Jugendlichen. Z. Entwicklungspsychol Pädagog Psychol, 19, 228248.

Welch, S.L. \& Fairburn, C.G. (1994): Sexual abuse and bulimia nervosa: three integrated case control comparisons. Am J Psychiat, 151: 402-406. 
Werner, E.E. \& Smith, R.S. (1982) : Vulnerable but invincible. A longitudinal study of resilient children and youth. New York: McGraw-Hill.

Werner, E.E. \& Smith, R.S. (1992): Overcoming the odds. High risk children from birth to adulthood. Ithaka, London: Cornell University Press.

Westen, D. Ludolph, P., Ruffins, S., Block, J. (1990): Physical and sexual abuse in adolescent girls with borderline personality disorder. Am J Orthopsychiat, 60: 55-66.

World Health Organization (1963-1965): Expert Committee on Dependenceproducing Drugs.

World Health Organization (1981): Memorandum Bulletin of the World Health Organization. Geneva, 59: 225-242.

Wyatt, G.E. \& Peters, S.D. (1986): Issues in the definition of child sexual abuse in prevalence research. Child Abuse and Neglect, 10, 231-240.

Yama et al. (1993): Childhood sexual abuse and parental alcoholism: interactive effects in adult women. Am Orthopsychiat Assoc. 63: 300-305.

Zanarini, M.C., Gunderson, J.G., Marino, F.M., Schwartz, E.O., Frankenburg, F.R. (1989): Childhood experiences of borderline patients. Comp Psychiat, 30: 18-25.

Zuravin, S.J. (1991): Research definitions of child abuse and neglect: Current problems. In: Starr, R. \& Wolfe, D. (eds): The effects of child abuse and neglect: Issues and research, Guildford Press, New York, 100-128. 


\section{Anhang}

\section{Anhang A: Tabellenverzeichnis}

Tabelle 1: Stichprobe nach Alter und Geschlecht

Tabelle 2: Stichprobe nach Wohnortgröße, Nationalität, Schulabschluss und Berufsausbildung 42

Tabelle 3: Familienstand und Wohnsituation 43

Tabelle 4: Sozialer Status der Herkunftsfamilie. 43

Tabelle 5: Familienbiographische Situation 44

Tabelle 6: Weitere familienbiographische Merkmale ..... 45

Tabelle 7: Prävalenz von familiärer Drogenabhängigkeit. 45

Tabelle 8: Häufigkeit verschiedener Missbrauchsformen..... 46

Tabelle 9: Lagemaße der Composite Scores des EuropASI nach allgemeiner Missbrauchserfahrung ....

Tabelle 10: Lagemaße der Skalenwerte des SCL 90-R nach allgemeiner

Missbrauchserfahrung ..... 48

Tabelle 11: Lagemaße der Globalen Kennwerte des SCL 90-R nach allgemeiner Missbrauchserfahrung 49

Tabelle 12: Lagemaße der Composite Scores des EuropASI nach sexueller Missbrauchserfahrung 50

Tabelle 13: Lagemaße der Skalenwerte des SCL 90-R nach sexueller Missbrauchserfahrung 51

Tabelle 14: Lagemaße der Globalen Kennwerte des SCL 90-R nach sexueller Missbrauchserfahrung ..... 52

Tabelle 15: Geschlechtsspezifische Unterschiede für die Composite Scores des EuropASI nach sexueller Missbrauchserfahrung .... 53

Tabelle 16: Geschlechtsspezifische Unterschiede für die Skalenwerten des SCL 90-R nach sexueller Missbrauchserfahrung. 
Tabelle 17: Geschlechtsspezifische Unterschiede für die Globalen Kennwerte des SCL 90-R nach sexueller Missbrauchserfahrung

Tabelle 18: Lagemaße der Composite Scores des EuropASI nach körperlicher Missbrauchserfahrung 54

Tabelle 19: Lagemaße der Skalenwerte des SCL 90-R nach körperlicher Missbrauchserfahrung 55

Tabelle 20: Lagemaße der Globalen Kennwerte des SCL 90-R nach körperlicher Missbrauchserfahrung ..... 56

Tabelle 21: Geschlechtsspezifische Unterschiede für die Composite Scores des

EuropASI nach körperlicher Missbrauchserfahrung.

Tabelle 22: Geschlechtsspezifische Unterschiede für die Skalenwerte des SCL

90-R nach körperlicher Missbrauchserfahrung

Tabelle 23: Geschlechtsspezifische Unterschiede für die Globalen Kennwerte des SCL 90-R nach körperlicher Missbrauchserfahrung.....

Tabelle 24: Lagemaße der Composite Scores des EuropASI nach

Vernachlässigung 58

Tabelle 25: Lagemaße der Skalenwerte des SCL 90-R nach Vernachlässigung...... 60

Tabelle 26: Lagemaße der Globalen Kennwerte des SCL 90-R nach

Vernachlässigung

Tabelle 27: Geschlechtsspezifische Unterschiede für die Composite Scores des EuropASI nach Vernachlässigung

Tabelle 28: Geschlechtsspezifische Unterschiede für die Skalenwerten des SCL 90-R nach Vernachlässigung.

Tabelle 29: Geschlechtsspezifische Unterschiede für die Globalen Kennwerte des SCL 90-R nach Vernachlässigung

Tabelle 30: Lagemaße der Composite Scores des EuropASI nach familiärer

Gewalt 62

Tabelle 31: Lagemaße der Skalenwerte des SCL 90-R nach familiärer Gewalt 64 Tabelle 32: Lagemaße der Globalen Kennwerte des SCL 90-R nach familiärer Gewalt. 65 
Tabelle 33: Geschlechtsspezifische Unterschiede für die Composite Scores des EuropASI nach familiärer Gewalt.

Tabelle 34: Geschlechtsspezifische Unterschiede für die Skalenwerten des SCL

90-R EuropASI nach familiärer Gewalt

Tabelle 35: Geschlechtsspezifische Unterschiede für die Globalen Kennwerte des SCL 90-R EuropASI nach familiärer Gewalt

Tabelle 36: Lagemaße der Composite Scores des EuropASI nach emotionaler Missbrauchserfahrung

Tabelle 37: Lagemaße der Skalenwerte des SCL 90-R nach emotionaler

Missbrauchserfahrung .... 68

Tabelle 38: Lagemaße der Globalen Kennwerte des SCL 90-R nach emotionaler

Missbrauchserfahrung ..... 69

Tabelle 39: Geschlechtsspezifische Unterschiede für die Composite Scores des

EuropASI nach emotionaler Misshandlung 69

Tabelle 40: Geschlechtsspezifische Unterschiede für die Skalenwerten des SCL

90-R nach emotionaler Misshandlung 70

Tabelle 41: Geschlechtsspezifische Unterschiede für die Globalen Kennwerte des SCL 90-R nach emotionaler Misshandlung.

Tabelle 42: Einfluss des Schweregrads des allgemeinen Missbrauchs auf die Composite Scores des EuropASI

Tabelle 43: Einfluss des Schweregrads des allgemeinen Missbrauchs auf die Skalenwerte des SCL 90-R.

Tabelle 44: Einfluss des Schweregrads des allgemeinen Missbrauchs auf die Globalen Kennwerte des SCL 90-R.

Tabelle 45: Einfluss des Schweregrads des sexuellen Missbrauchs auf die

Composite Scores des EuropASI 74

Tabelle 46: Einfluss des Schweregrads des sexuellen Missbrauchs auf die

Skalenwerte des SCL 90-R 75

Tabelle 47: Einfluss des Schweregrads des sexuellen Missbrauchs auf die Globalen Kennwerte des SCL 90-R. 76 
Tabelle 48: Einfluss des Schweregrads der körperlichen Gewalt auf die

Composite Scores des EuropASI

Tabelle 49: Einfluss des Schweregrads der körperlichen Gewalt auf die

Skalenwerte des SCL 90-R

Tabelle 50: Einfluss des Schweregrads der körperlichen Gewalt auf die Globalen

Kennwerte des SCL 90-R....

Tabelle 51: Einfluss des Schweregrads der Vernachlässigung auf die Composite

Scores des EuropASI.

Tabelle 52: Einfluss des Schweregrads der Vernachlässigung auf die

Skalenwerte des SCL 90-R

Tabelle 53: Einfluss der Schweregrade der Vernachlässigung auf die Globalen

Kennwerte des SCL 90-R 82

Tabelle 54: Einfluss des Schweregrads der familiären Gewalt auf die Composite

Scores des EuropASI. 83

Tabelle 55: Einfluss des Schweregrads der familiären Gewalt auf die

Skalenwerte des SCL 90-R 85

Tabelle 56: Einfluss des Schweregrads der familiären Gewalt auf die Globalen Kennwerte des SCL 90-R

Tabelle 57: Einfluss des Schweregrads des emotionalen Missbrauchs auf die Composite Scores des EuropASI

Tabelle 58: Einfluss des Schweregrads des emotionalen Missbrauchs auf die Skalenwerte des SCL 90-R.

Tabelle 59: Einfluss des Schweregrads des emotionalen Missbrauchs auf die Globalen Kennwerte des SCL 90-R. 90

Tabelle 60: Vergleich der Mittelwerte der Composite Scores für die verschiedenen Missbrauchsgruppen

Tabelle 61: Vergleich der Mittelwerte der Skalenwerte für die verschiedenen

Missbrauchsgruppen, die Gruppe ohne Missbrauch und die gesamte Stichprobe ... 93

Tabelle 62: Vergleich der Mittelwerte der drei globalen Kennwerte für die verschiedenen Missbrauchsgruppen, die Gruppe ohne Missbrauch und die gesamte Stichprobe. 
Tabelle 63: Signifikante Composite Scores nach EuropASI für die verschiedenen Missbrauchsgruppen

Tabelle 64: Signifikante Kennwerte nach SCL 90-R für die verschiedenen

Missbrauchsgruppen 97

Tabelle 65: Kombinationen der Missbrauchserfahrungen. 103

Tabelle 66: Vergleich der Mittelwerte der Skalenwerte für die verschiedenen

Missbrauchsgruppen, die Gruppe ohne Missbrauch und die gesamte Stichprobe . 107 Tabelle 67: Referenzwerte nach Franke für Normal Gesunde, HIV infizierte, substanzabhängige und schizophrene Patienten

Tabelle 68: Signifikante Composite Scores nach EuropASI bei einer

Unterscheidung des Schweregrads des Missbrauchs

Tabelle 69: Signifikante Kennwerte nach SCL 90-R bei einer Unterscheidung des

Schweregrads des Missbrauchs

Tabelle 70: Signifikante Composite Scores nach EuropASI bei einer Unterscheidung nach Geschlechtern.

Tabelle 71: Signifikante Kennwerte nach SCL 90-R bei einer Unterscheidung nach Geschlechtern.

Tabelle 72: Vergleich der Mittelwerte der Skalenwerte für die verschiedenen Missbrauchsgruppen, die Gruppe ohne Missbrauch und die gesamte Stichprobe 125 Tabelle 73: Referenzwerte nach Franke für Normal Gesunde, HIV infizierte, substanzabhängige und schizophrene Patienten 126

Tabelle 74: Übereinstimmung der Aussagen zu Missbrauchserfahrungen $(n=83)$. 130 


\section{Anhang B: Münchener Traumafragebogen}

\section{Münchener Traumafragebogen}

Martin Schäfer, Marion Herpers, Isabelle Rassaerts, Michael Soyka;

München 1999

Psychiatrische Klinik der LMU, Nußbaumstraße 7,

D-80336 München

Definitionen modifiziert nach:

Robin A. McGee, David A. Wolfe, Sandra A. Yuen, Susan K. Wilson, Jean Carnochan: The measurement of maltreatment: A comparison of approaches Child Abuse \& Neglect, Vol. 19, No. 2, pp. 233-249, 1995 


\section{Allgemeine Angaben}

1. Alter:

2. Geschlecht: weiblich männlich

3. Staatsangehörigkeit:

4. Geburtsland:

von Ihnen

Ihres Vaters

Ihrer Mutter

5. Manche Menschen wachsen ihre gesamte Kindheit und Jugend über bei ihren leiblichen Eltern auf. Viele leben jedoch auch nur mit einem Elternteil zusammen, haben Stiefeltern, leben in Pflegefamilien oder Heimen. Wo sind Sie bis zu ihrem 6.Lebensjahr und nach ihrem 6.Lebensjahr aufgewachsen?

( Bitte jeweils nur eine Antwort ankreuzen )

\section{Lebensjahr}

bis zum 6.Lebensjahr nach dem

bei den leiblichen Eltern

bei der leiblichen Mutter 
bei dem leiblichen Vater

bei der leiblichen Mutter und Stiefvater

bei dem leiblichen Vater und Stiefmutter

bei den Großeltern

bei sonstigen Verwandten

bei Adoptiveltern

bei Pflegeeltern

in einem Heim

6. Waren Sie vom 1. bis zum 6.Lebensjahr über einen längeren Zeitraum von ihren Eltern getrennt?

nein ja

7. Waren Sie ein Wunschkind?

nein ja

8. Haben Sie Geschwister?

nein ja

Wenn ja, geben Sie bitte an, wieviele Schwestern oder Brüder Sie haben:

Schwestern _ Brüder 
9. In welche Berufsgruppe würden Sie ihre Eltern einordnen?

( Bitte nur eine Antwort ankreuzen )

Arbeiter / niedriges Einkommen

Angestellte / mittleres Einkommen

Angstellte / hohes Einkommen

Selbständige / mittleres Einkommen

Selbstständige / hohes Einkommen

Selbstständige / sehr hohes Einkommen 


\section{Fragen zu Mißbrauchserfahrungen}

Wir bitten Sie nun, uns einige Fragen zu sexuellem Mißbrauch, physischer Mißhandlung und Vernachlässigung zu beantworten. Wir sind uns darüber im klaren, daß es für Sie nicht einfach ist, über solch belastende Erlebnisse Auskunft zu geben. Wir wissen ihre Teilnahme zu schätzen und sind Ihnen sehr dankbar dafür! Desweiteren können Sie versichert sein, daß ihre Angaben striktest vertraulich behandelt werden. Sie bleiben völlig anonym und können mit Ihrer Person nicht in Verbindung gebracht werden.

Um Ihnen das Beantworten der Fragen zu erleichtern, möchten wir vorab noch einiges anmerken:

In den folgenden Fragen sind der sexuelle Mißbrauch, die physische Mißhandlung sowie die Vernachlässigung im einzelnen nochmals genau definiert.

Bitte beachten Sie, daß sich die Fragen auf von Ihnen nicht gewollte, durch andere erzwungene Handlungen in ihrer Kindheit bis hin zu ihrem 14.Lebensjahr beziehen. Späterer sexueller Kontakt im Rahmen einer Partnerbeziehung im Erwachsenenalter soll hiermit nicht erfragt werden.

Für die Auswertung der Studie allgemein ist es von großer Bedeutung, daß Sie die Fragen vollständig beantworten, so gut Sie dies können. Sollten Sie eine der Fragen nicht verstehen oder mit den vorgegebenen Antwortmöglichkeiten Schwierigkeiten haben, so teilen sie uns dies bitte am Rand oder auf der Rückseite dieses Bogens mit. Wenn Sie sich an bestimmte Erlebnisse nicht erinnern können, so formulieren Sie dies bitte ebenfalls kurz am Rand oder auf der Rückseite des Bogens. Bitte bedenken Sie jedoch, daß wir nur vollständig ausgefüllte Fragebögen auswerten können. Lediglich eine möglichst ehrliche Beantwortung aller Fragen ermöglicht uns eine Aussage über belastende Ereignisse in ihrer Kindheit und mögliche Zusammenhänge mit ihrer Suchtsituation.

Vielen Dank für ihre Bemühungen! 
Abschnitt I:

1. Haben Sie als Kleinkind, Kind oder heranwachsender Jugendlicher bis zum 14.Lebensjahr Situationen erlebt, in denen eine jugendliche oder eine erwachsene Person Sie gegen Ihren Willen zu sexuellen Handlungen verführt, genötigt oder gezwungen hat?

nein ja

Wenn ja, würden wir Sie bitten die nachfolgenden Fragen zu beantworten.

Wenn nein, dann fahren Sie bitte mit den unter Abschnitt II aufgeführten Fragen (siehe Seite 10) fort.

1.1 Beurteilen Sie zunächst bitte die Schwere der von Ihnen erlebten Situation auf der angegebenen Skala von 1 bis 10 ( 1 steht für leicht, 5 für mittel, 10 für schwer).

Kreuzen Sie hierzu bitte die entsprechende Zahl an.

$$
1-2-3-4-5-6-7-8-9-10
$$

1.2Wie alt waren Sie, als dies zum ersten Mal geschah?

( wenn genaues Alter bekannt, bitte eintragen )

jünger als 6 Jahre

6 bis 10 Jahre

10 bis 14 Jahre

1.3Wer war die Person?

Vater Mutter Geschwister 


$\begin{array}{lll}\text { Stiefvater } & \text { Stiefmutter } & \text { Verwandte } \\ \text { Großvater } & \text { Großmutter } & \text { Freunde / Bekannte } \\ \text { Fremde } & & \end{array}$

1.4 Alter dieser Person / Personen:

jünger als 14 Jahre

14 bis 18 Jahre

18 bis 21 Jahre

21 bis 40 Jahre

40 bis 60 Jahre

60 bis 80 Jahre

1.5Wurde von der Person Gewalt ausgeübt?

nein ja

2. Haben Sie sich als Kind sexuell beobachten lassen müssen? Mußten Sie z.B. nackt tanzen, wurden Sie nackt fotografiert oder unter der Dusche beobachtet oder hat man Sie zu sexuellen Spielen mit ihren Geschwistern ermutigt oder Ähnliches?

nein ja

Wenn ja, würden wir Sie bitten die nachfolgenden Fragen zu beantworten.

Wenn nein, dann fahren Sie bitte mit der unter 3. aufgeführten Frage ( siehe Seite 6 ) fort. 
2.1 Wie alt waren Sie, als dies zum ersten Mal geschah?

( wenn genaues Alter bekannt, bitte eintragen )

jünger als 6 Jahre

6 bis 10 Jahre

10 bis 14 Jahre

2.2 Wer hat Sie dazu veranlaßt?

2.3Wie oft haben Sie diese Situationen erlebt?

ein Mal

alle paar Monate

alle paar Tage

beinahe jeden Tag

2.4 Hat die Person Sie dazu gezwungen oder körperliche Gewalt ausgeübt?

nein

ja

2.5Wie alt waren Sie, als diese Übergriffe endeten?

2.6 Glauben Sie, daß das Ereignis Einfluß darauf hatte, wie Sie bisher mit ihrem Leben zurechtgekommen sind?

nein ja 
2.7 Glauben Sie, daß zwischen ihrer jetzigen Sucht und dem damaligen Ereignis ein Zusammenhang besteht?

nein ja

3. Wurden Sie als Kind gegen ihren Willen sexuell berührt, angefaßt oder gestreichelt, so daß es Ihnen unangenehm war? Hat eine erwachsene Person an ihren Geschlechtsorganen herumgespielt oder mußten sie die Geschlechtsorgane dieser Person berühren? Und hat sich dabei die erwachsene Person sexuell erregt oder befriedigt?

nein ja

Wenn ja, würden wir Sie bitten die nachfolgenden Fragen zu beantworten.

Wenn nein, dann fahren Sie bitte mit der unter 4. aufgeführten Frage ( siehe Seite 8 ) fort.

3.1 Wie alt waren Sie, als dies zum ersten Mal geschah?

( wenn genaues Alter bekannt, bitte eintragen )

jünger als 6 Jahre

6 bis 10 Jahre

10 bis 14 Jahre

3.2 Wer hat Sie dazu veranlaßt? 
3.3Wie oft haben Sie diese Situationen erlebt?
ein Mal
alle paar Monate
alle paar Tage
beinahe jeden Tag

3.4 Hat die Person Sie dazu gezwungen oder körperliche Gewalt ausgeübt?

nein ja

3.5Wie alt waren Sie, als diese Übergriffe endeten?

3.6 Glauben Sie, daß das Ereignis Einfluß darauf hatte, wie Sie bisher mit ihrem Leben zurechtgekommen sind?

nein ja

3.7 Glauben Sie, daß zwischen ihrer jetzigen Sucht und dem damaligen Ereignis ein Zusammenhang besteht?

nein ja

4. Wurden Sie als Kind sexuell gegen ihren Willen in schwerer Form von einer erwachsenen Person benutzt, d.h. durch vollzogenen Geschlechtsverkehr mit oraler, vaginaler oder analer Penetration?

nein ja 
Wenn ja, würden wir Sie bitten die nachfolgenden Fragen zu beantworten.

Wenn nein, dann fahren Sie bitte mit der unter 5. aufgeführten Frage ( siehe Seite 9 ) fort.

4.1 Wie alt waren Sie, als dies zum ersten Mal geschah?

( wenn genaues Alter bekannt, bitte eintragen )

jünger als 6 Jahre

6 bis 10 Jahre

10 bis 14 Jahre

4.2Wer hat Sie dazu veranlaßt?

4.3Wie oft haben Sie diese Situationen erlebt?

ein Mal

alle paar Monate

alle paar Tage

beinahe jeden Tag

4.4 Hat die Person Sie dazu gezwungen oder körperliche Gewalt ausgeübt?

nein

ja

4.5Wie alt waren Sie, als diese Übergriffe endeten? 
4.6Glauben Sie, daß das Ereignis Einfluß darauf hatte, wie Sie bisher mit ihrem Leben zurechtgekommen sind?

nein

ja

4.7 Glauben Sie, daß zwischen ihrer jetzigen Sucht und dem damaligen Ereignis ein Zusammenhang besteht?

nein ja

5. Waren Sie jemals zum Zeitpunkt eines sexuellen Mißbrauchs ( Bitte nur eine Antwort ankreuzen )

alkoholisiert ( selbst eingenommen )

alkoholisiert ( durch Täter eingeflößt )

unter Drogen ( selbst eingenommen )

unter Drogen ( durch Täter eingeflößt )

nein

Wenn ja, welchen Effekt hatte das auf Sie?

positiv negativ keinen

6. War die Person alkoholisiert?

nein ja

7. War die Person unter Drogen?

nein ja 
Abschnitt II:

1. Sind Sie aus ihrer Sicht als Kind körperlich mißhandelt worden?

nein ja

1.1 Wenn ja, beurteilen Sie zunächst bitte die Schwere der von Ihnen erlebten Situation auf der angegebenen Skala von 1 bis 10 ( 1 steht für leicht, 5 für mittel, 10 für schwer ).

Kreuzen Sie hierzu bitte die entsprechende Zahl an.

$$
1-2-3-4-5-6-7-8-9-10
$$

2. Haben Sie aus Ihrer Sicht als Kind ungerechtfertigt viele Ohrfeigen und Schläge bekommen?

nein ja

3. Haben Sie aus ihrer Sicht brutale Situationen als Kind erlebt, in denen Sie gesundheitlich gefährdet waren ( wurden Sie z.B. geschüttelt, Gegenstände nach Ihnen geworfen oder wurden Sie über den Balkon gehalten mit der Drohung losgelassen zu werden )?

nein ja

4. Haben Sie als Kind Schäden durch körperliche Mißhandlung erlitten ( blaue Flecken, Knochenbrüche oder andere Verletzungen infolge von

Faustschlägen, Stockschlägen, Verbrennungen, Würgen, gegen die Wand Stoßen oder durch Waffengewalt, z.B. Messer )

nein ja 
5. War die ausführende Person häufig alkoholisiert?

nein ja

5.1War die ausführende Person häufig unter Drogen?

nein ja

5.2Wie alt waren Sie, als dies zum ersten Mal geschah?

( wenn genaues Alter bekannt, bitte eintragen )

jünger als 6 Jahre

6 bis 10 Jahre

10 bis 14 Jahre

5.3Wie oft haben Sie diese Situationen erlebt?

ein Mal

alle paar Monate

alle paar Tage

beinahe jeden Tag

5.4Wie alt waren Sie, als diese Übergriffe endeten? 
5.5Glauben Sie, daß das Ereignis Einfluß darauf hatte, wie Sie bisher mit ihrem Leben zurechtgekommen sind?

nein ja

5.6 Glauben Sie, daß zwischen ihrer jetzigen Sucht und dem damaligen Ereignis ein Zusammenhang besteht?

nein ja 
Abschnitt III:

1. Haben Sie das Gefühl, daß Sie als Kind in ihrer Familie vernachlässigt wurden?

nein ja

1.1 Wenn ja, beurteilen Sie zunächst bitte die Schwere der von Ihnen erlebten Situation auf der angegebenen Skala von 1 bis 10 ( 1 steht für leicht, 5 für mittel, 10 für schwer ).

Kreuzen Sie hierzu bitte die entsprechende Zahl an.

$$
1-2-3-4-5-6-7-8-9-10
$$

2. Haben Sie den Eindruck, daß Ihnen in ihrer Kindheit durch die

Erziehungsberechtigten

( Eltern, Stiefeltern, usw. ) wenig Aufmerksamkeit, Lob und Trost zuteil wurde?

Haben ihre Eltern wenig Zeit mit Ihnen verbracht und Sie selten zu Aktivitäten ( z.B. zum Spielen mit Gleichaltrigen ) aufgefordert?

Fanden Sie wenig Unterstützung durch ihre Eltern und wurde ihre Meinung selten respektvoll beachtet?

( Antworten Sie bitte nur mit ja, wenn die meisten dieser Punkte auf Sie zutreffen )

nein ja

3. Haben Sie den Eindruck, daß ihre Entwicklung durch die

Erziehungsberechtigten

( Eltern, Stiefeltern, usw. ) nicht ausreichend gefördert wurde?

Würden Sie sagen, daß Sie keine angemessene Erziehung erhalten haben?

Wurde Ihnen selten Ansporn zuteil?

Wurden Sie in der Schule unzureichend gefördert? Wurden ihre schulischen Leistungen nicht kontrolliert?

Gab es in ihrer Kindheit keine beständigen Regeln, die es zu befolgen galt?

( Antworten Sie bitte nur mit ja, wenn die meisten dieser Punkte auf Sie zutreffen ) 
nein

ja

4. Fühlten Sie sich in ihrer familiären Umgebung selten geborgen?

Haben Sie den Eindruck, daß Sie in ihrer Kindheit oft kein Essen bekommen haben, daß Ihnen nötige Medikamente oder Arztbesuche vorenthalten wurden?

Wurden Sie äußerlich vernachlässigt, wurde zu wenig auf ihre Körperpflege und ihre Kleidung geachtet?

Sind Sie als Kind oft alleine gelassen worden und fanden Sie nur selten in gefährlichen Situationen Schutz bei ihren Eltern?

( Antworten Sie bitte nur mit ja, wenn die meisten dieser Punkte auf Sie zutreffen )

nein ja

5. Glauben Sie, daß das Ereignis Einfluß darauf hatte, wie Sie bisher mit ihrem Leben zurechtgekommen sind?

nein ja

5.1 Glauben Sie, daß zwischen ihrer jetzigen Sucht und dem damaligen Ereignis ein Zusammenhang besteht?

nein ja 
Abschnitt IV:

1. Sind Sie früher gehäuft innerhalb der Familie mit Gewalt zwischen den Erziehungsberechtigten konfrontiert worden?

nein ja

1.1 Wenn ja, beurteilen Sie bitte die Schwere der von Ihnen erlebten Situationen auf der angegebenen Skala von 1 bis10 (1 steht für leicht, 5 für mittel, 10 für schwer). Kreuzen Sie hierzu bitte die entsprechende Zahl an.

$$
1-2-3-4-5-6-7-8-9-10
$$

2. Haben Sie sehr oft Streitereien zwischen den Erziehungsberechtigten miterlebt, bei denen es zu harten verbalen Auseinandersetzungen kam, Gegenstände zerschlagen wurden, jedoch keine Schläge zwischen den beteiligten Personen erfolgten, für Sie jedoch diese Situation sehr bedrohlich und beängstigend wirkte?

nein ja

3. Haben Sie sehr oft Streitereien zwischen den Erziehungsberechtigten miterlebt, bei denen es zu körperlichen Auseinandersetzungen kam, wie Ohrfeigen, Gegenstände nach dem Partner werfen oder inn schubsen ohne körperliche Verletzungen?

nein ja 
4. Haben Sie sehr oft Streitereien zwischen den Erziehungsberechtigten miterlebt, bei denen es zu massiver körperlicher Bedrohung einer Person kam, z.B. durch Faustschlag, Würgen, Schlag mit Gegenständen ( Holz, Eisen ) oder durch den Einsatz anderer Waffen?

nein ja

5. Glauben Sie, daß das Ereignis Einfluß darauf hatte, wie Sie bisher mit ihrem Leben zurechtgekommen sind?

nein ja

5.1 Glauben Sie, daß zwischen ihrer jetzigen Sucht und dem damaligen Ereignis ein Zusammenhang besteht?

nein ja 
Abschnitt $\mathrm{V}$ :

1. Sind Sie aus Ihrer Sicht in Ihrer Kindheit emotional ( gefühlsmäßig ) mißhandelt worden?

nein ja

1.1 Wenn ja, beurteilen Sie bitte die Schwere der von Ihnen erlebten Situationen auf der angegebenen Skala von 1 bis10 ( 1 steht für leicht, 5 für mittel, 10 für schwer ). Kreuzen Sie hierzu bitte die entsprechende Zahl an.

$$
1-2-3-4-5-6-7-8-9-10
$$

2. Haben Sie das Gefühl, daß Sie als Kind oft kritisiert und gekränkt wurden? Sind ihre Gefühle und Probleme häufig ignoriert worden?

Wurde Ihnen damals wenig Gefühl von ihren Erziehungsberechtigten entgegengebracht?

Haben die Erziehungsberechtigten nur wenig mit Ihnen gesprochen?

Waren, laut ihren Erziehungsberechtigten, andere immer besser als Sie?

( Antworten Sie bitte nur mit ja, wenn die meisten dieser Punkte auf Sie zutreffen )

nein ja

3. Haben Sie das Gefühl, daß Sie als Kind von ihren Erziehungsberechtigten oft verspottet und lächerlich gemacht wurden oder daß über Sie sarkastisch oder ironisch gesprochen wurde?

Wurden ihre Aussagen ständig als falsch oder als Lügen dargestellt?

Wurden Sie häufig für Probleme innerhalb der Familie verantwortlich gemacht?

Wußten Sie oft nie, wie die Erziehungsberechtigten reagieren würden und wurde oft mit Ihnen in einem feindseeligen Ton gesprochen?

Sind Sie oft emotionalen Ausbrüchen der Erziehungsberechtigten ausgesetzt gewesen?

Wurden Sie je kriminellen Einflüssen ausgesetzt?

( Antworten Sie bitte nur mit ja, wenn die meisten dieser Punkte auf Sie zutreffen ) 
nein

ja

4. Haben Sie das Gefühl, daß Sie von ihren Eltern als unerwünscht angesehen wurden oder als Ursache aller Probleme?

Wurde je durch ihre Erziehungsberechtigten eine für Sie äußerst wertvolle Sache ( z.B. ihr Lieblingsstofftier ) zerstört?

Haben Sie den Eindruck, daß ihre Erziehungsberechtigten vorsätzlich versuchten Ihnen Angst einzujagen?

Wurde Ihnen oft mit extremen Bestrafungen gedroht oder wurde Ihnen angedroht Sie zu verlassen oder zu töten?

( Antworten Sie bitte nur mit ja, wenn die meisten dieser Punkte auf Sie zutreffen )

nein ja

5. Glauben Sie, daß das Ereignis Einfluß darauf hatte, wie Sie bisher mit ihrem Leben zurechtgekommen sind?

nein ja

5.1 Glauben Sie, daß zwischen ihrer jetzigen Sucht und dem damaligen Ereignis ein Zusammenhang besteht?

nein ja 


\section{Anhang C: Danksagung}

Ich danke Prof. Dr. med. H.-J. Möller und meinem Doktorvater Prof. Dr. med. M. Soyka für die wohlwollende Überlassung des Themas. Besonders gilt mein Dank Dr. med. M. Schäfer, der maßgeblich an der Entwicklung des Traumafragebogens und Ausgestaltung des Themas beteiligt war und darüber hinaus mir als Betreuer jederzeit mit seiner konstruktiven Kritik, seinen Anregungen und Hinweisen die notwendige sachliche und fachliche Unterstützung zukommen ließ sowie den Fortgang meiner Arbeit auch über seinen Wechsel von der Universitätsklinik München an die Charité Berlin begleitete.

Außerdem geht mein Dank an die Patienten, die an der Studie teilnahmen und ohne deren Bereitschaft diese Arbeit nicht durchführbar gewesen wäre. Ebenso geht mein Dank an die Leiter und Mitarbeiter der psychiatrischen Kliniken in München, Augsburg, Aachen und Lübeck, die mich bei der Durchführung der Studie sehr unterstützten.

Ganz besonderer Dank gilt Frau Isabelle Rassaerts, die für den anderen Teil der Studie bezüglich alkoholabhängigen Patienten verantwortlich war und mit der ich gemeinsam die interessante, aber häufig auch herausfordernde Erhebung der Daten durchgeführt habe. Gerade in schwierigen Zeiten verstand sie es durch ihre besondere Art mir die notwendige Kraft zu schenken.

Darüber hinaus gilt mein Dank meinem Ehemann Dominik Schnieders, der nicht nur mein Ansprechpartner für technische und formale Probleme war, sondern mir auch durch seine tägliche Aufmunterung über die Höhen und Tiefen des Entstehungsprozesses hinweghalf. 
Nicht zuletzt danke ich meinen Eltern, Helma und Stefan Herpers, die mir meine Ausbildung ermöglichten und mich stets verständnisvoll begleiteten.

München, 2004

Marion Schnieders 